\title{
Towards an integrated modeling of the plasma-solid interface
}

\author{
M Bonitz ${ }^{1}$, A Filinov ${ }^{1,2,3}$, J W Abraham ${ }^{1}, \mathrm{~K}_{\text {Balzer }}^{4}$, H Kählert $^{1}$, E Pehlke ${ }^{1}$, \\ FX Bronold ${ }^{5}$, M Pamperin ${ }^{5}$, M M Becker ${ }^{2}$, D Loffhagen ${ }^{2}, \mathrm{H}_{\text {Fehske }}{ }^{5}$ \\ ${ }^{1}$ Institut für Theoretische Physik und Astrophysik, Christian-Albrechts-Universität, Leibnizstr. 15, D-24098 Kiel, Germany \\ ${ }^{2}$ Leibniz-Institut für Plasmaforschung und Technologie e.V. (INP), Felix-Hausdorff-Str. 2, D-17489 Greifswald, Germany \\ 3 Joint Institute for High Temperatures RAS, Izhorskaya Str. 13, 125412 Moscow, Russia \\ ${ }^{4}$ Rechenzentrum der Christian-Albrechts-Universität Kiel and \\ ${ }^{5}$ Institut für Physik, Universität Greifswald
}

\begin{abstract}
Solids facing a plasma are a common situation in many astrophysical systems and laboratory setups. Moreover, many plasma technology applications rely on the control of the plasma-surface interaction, i.e. of the particle, momentum and energy fluxes across the plasma-solid interface. However, presently often a fundamental understanding of them is missing, so most technological applications are being developed via trial and error. The reason is that the physical processes at the interface of a low-temperature plasma and a solid are extremely complex, involving a large number of elementary processes in the plasma, in the solid as well as fluxes across the interface. An accurate theoretical treatment of these processes is very difficult due to the vastly different system properties on both sides of the interface: quantum versus classical behavior of electrons in the solid and plasma, respectively; as well as the dramatically differing electron densities, length and time scales. Moreover, often the system is far from equilibrium. In the majority of plasma simulations surface processes are either neglected or treated via phenomenological parameters such as sticking coefficients, sputter rates or secondary electron emission coefficients. However, those parameters are known only in some cases and with very limited accuracy. Similarly, while surface physics simulations have often studied the impact of single ions or neutrals, so far, the influence of a plasma medium and correlations between successive impacts have not been taken into account. Such an approach, necessarily neglects the mutual influences between plasma and solid surface and cannot have predictive power.

In this paper we discuss in some detail the physical processes a the plasma-solid interface which brings us to the necessity of coupled plasma-solid simulations. We briefly summarize relevant theoretical methods from solid state and surface physics that are suitable to contribute to such an approach and identify four methods. The first are mesoscopic simulations such as kinetic Monte Carlo (KMC) and molecular dynamics (MD) that are able to treat complex processes on large scales but neglect electronic effects. The second are quantum kinetic methods based on the quantum Boltzmann equation that give access to a more accurate treatment of surface processes using simplifying models for the solid. The third approach are $a b$ initio simulations of surface process that are based on density functional theory (DFT) and time-dependent DFT. The fourths are nonequilibrium Green functions that able to treat correlation effects in the material and at the interface. The price for the increased quality is a dramatic increase of computational effort and a restriction to short time and length scales. We conclude that, presently, none of the four methods is capable of providing a complete picture of the processes at the interface. Instead, each of them provides complementary information, and we discuss possible combinations.
\end{abstract}

PACS numbers:

\section{INTRODUCTION}

Modern plasma physics has three main research topics [1]: high-temperature plasmas, in particular magnetic fusion; high-density plasmas ("warm dense matter", laser plasmas, inertial confinement fusion); and low-temperature plasmas. The location of these areas in the density-temperature plain is sketched in Fig. 1. In each of these fields, the processes at the plasma wall play a crucial role, both, for fundamental understanding and for technological applications. Therefore, progress in the simulation of plasma-solid interaction is of crucial importance in each of these fields. Here we concentrate on plasma-solid processes in low-temperature plasma, although some of our results are expected to be of interest also for the high-temperature plasmas. This field has experienced impressive progress over the last two decades, both, in experiments and applications.

Aside from traditional applications also new materials, in particular nanomaterials, are coming into the focus [2,3].
This includes carbon based materials such as carbon nanotubes and graphene nanoribbons that have a size-dependent bandgap and promise exciting electronic and optical properties, e.g. $[4,5]$. The role of plasmas, in the context of these novel materials, is only poorly explored yet. There are impressive first results on plasma synthesis of these systems. On the other hand, it will also be interesting to use such materials inside a plasma and to utilize their properties in a discharge environment.

These applications are only emerging, and a brief discussion of some aspects will be given in this paper. Yet for their success, and for solids embedded in plasmas in general, it will be crucial to have available accurate simulations of the plasma-solid interface, as has been pointed out in many places, e.g. [1,6]. The interest in low-temperature plasmas arises from the peculiar properties of these systems. These plasmas typically have a low degree of ionization and cover a broad pressure range, from below one $\mathrm{Pa}$ to atmospheric pressure, see Fig. 2. These plasmas are non-thermal, i.e. the electron and ion temperatures may differ by several orders of 


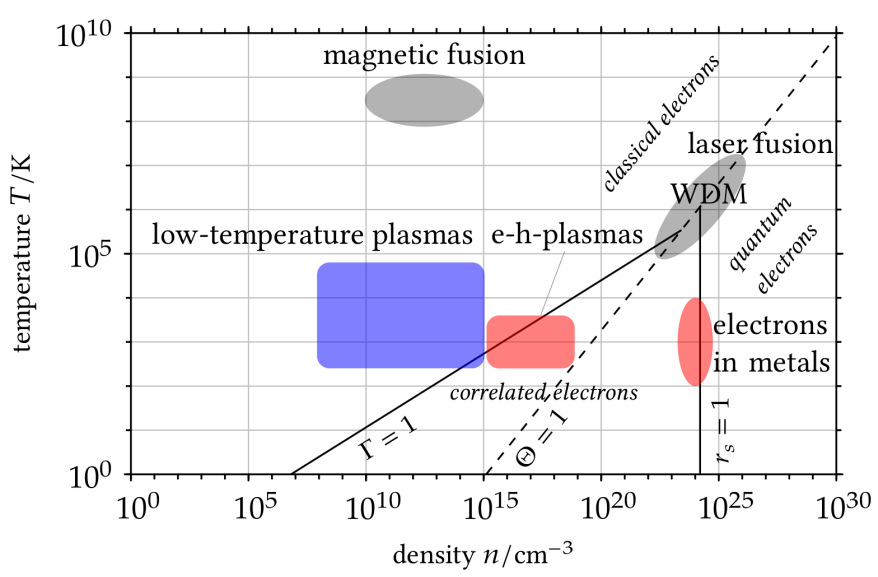

Figure 1: Low-temperature plasmas (LTP) are one of three main current research topics in plasma physics, aside from magnetic fusion and dense plasma (warm dense matter, WDM) ${ }^{1}$. These systems cover a huge parameter range in the density-temperature plane. LTP (the blue box) range from low (electron) density to atmospheric pressure (right edge). Representatives of solids facing the plasma are metals and semiconductors (electron-hole plasmas) sketched by the red areas. Relevant dimensionless parameters are the classical coupling parameter, $\Gamma=e^{2} / \bar{r} k_{B} T$, the quantum coupling parameter, $r_{s}=\bar{r} / a_{B}$ and the degeneracy parameter of the electrons, $\Theta=k_{B} T / E_{F}$, with $\bar{r}, a_{B}$ and $E_{F}$ denoting the mean interparticle distance, the Bohr radius and the Fermi energy, respectively.

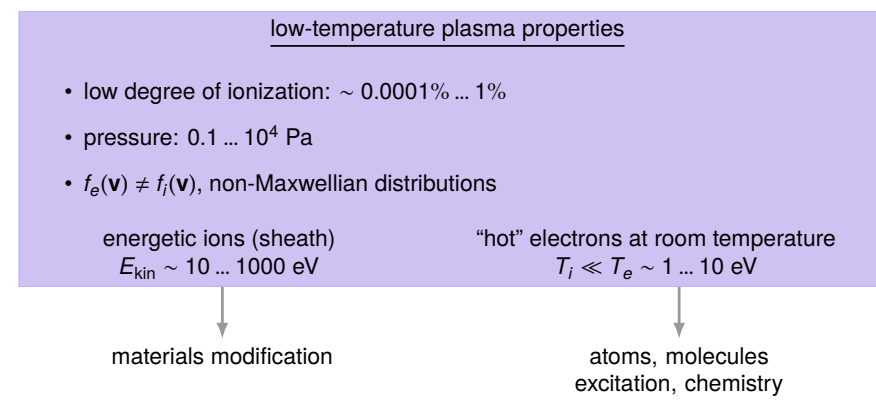

Figure 2: Low-temperature plasmas being composed of neutrals (atoms, molecules), ions and electrons comprise a number of very unusual properties: they are non-isothermal $\left(T_{i} \neq T_{e}\right)$, far from thermal equilibrium (non-Maxwellian velocity distributions), and they contain electrons and ions of a very broad range of kinetic energies. In the plasma boundary region ("sheath") ions may reach high energies that can be exploited for materials modification, sputtering and ion implantation. At the same time, electrons with energies in the $\mathrm{eV}$ range are able to excite and ionize neutrals and trigger chemical reactions.

magnitude. Moreover, electrons and ions may be far from thermal equilibrium, being described by non-Maxwellian velocity distributions. The existence of energetic electrons with temperatures in the eV-range, which is sufficient to excite or ionize atoms and molecules, is of high interest for applications in surface chemistry, biology and even medicine. On the other hand, these plasmas may contain highly energetic ions that are accelerated by strong electric fields, in particular in the surface near region (the "plasma sheath"). These energies are sufficient for mechanical modification of the solid surface such as defect creation, ion implantation or sputtering.

Even though there have been remarkable recent advances, both, in plasma modeling and surface science simulations, the combination of the two is still at a very early stage. Current simulations in low-temperature plasma physics that are based on kinetic theory or fluid theory have achieved a high quality description of the dynamics of electrons, ions and neutrals in the plasma bulk, including elastic and inelastic scattering processes, cf. top part of Fig. 3. At the same time, these simulations often omit plasma-surface processes or treat them phenomenologically. For example, in many advanced kinetic
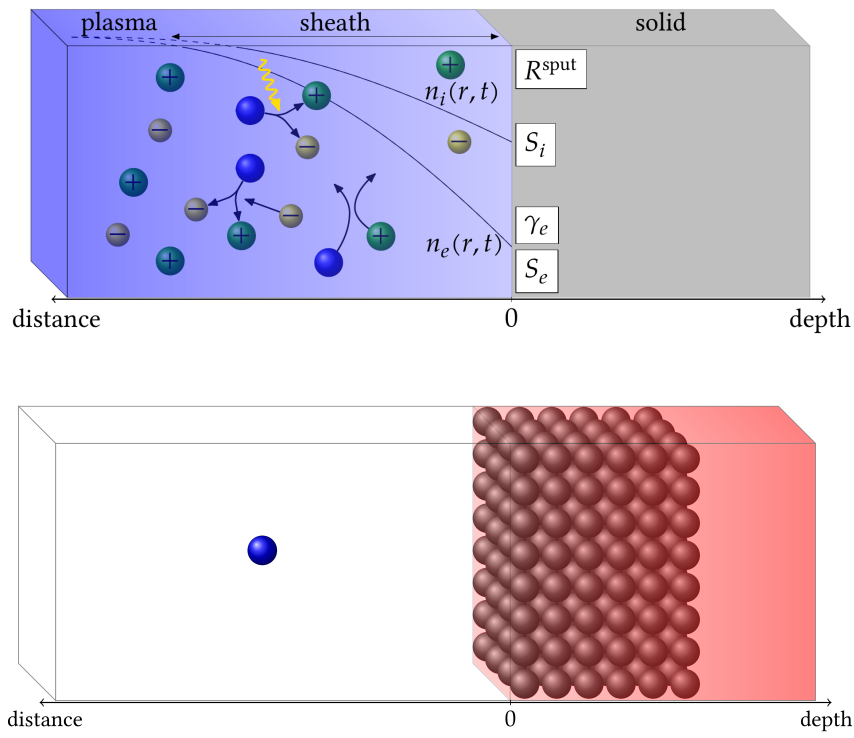

Figure 3: Top: Sketch of the current approach to include surface properties in plasma simulations via phenomenological parameters such as sputter rates $R^{\text {sput }}$, electron and ion sticking coefficients, $S_{e}, S_{i}$ and the secondary electron emission coefficient, $\gamma_{e}$. While the plasma is treated by advanced approaches, the atomic structure of the solid and the surface is not resolved. Bottom: In contrast, in surface science, atomic level information of the surface is taken into account, whereas plasma effects are approximated via independent impacts of neutrals or ions.

simulations based on the Boltzmann equation, e.g. [7,8] or particle in cell (PIC-MCC) simulations, e.g. [9,10] neutrals are treated as a homogeneous background, and their interaction with surfaces is not included in the description. However, the effect of energetic neutrals maybe crucial for secondary electron emission (SEE), as was demonstrated in PIC simulations of Derszi et al. [11]. Another effect that can be important for the behavior of the plasma are the properties of the surface, such as surface roughness, oxidation or coverage by an adsorbate. For example, Phelps and Petrovic [12] convincingly demonstrated that the plasma modification of a metal surface may change SEE by several orders of magnitude, see Sec. II. The conclusion is that an accurate theoretical treatment of 
the solid surface may be very important for low-temperature plasma simulations. This suggests to resort to surface science methods where a very accurate atomic level treatment of solid surfaces has been achieved by ab initio methods such as density functional theory (DFT). Surface simulations have also incorporated the impact of energetic projectiles to simulate sputtering, e.g. [13] or energy loss (stopping power), e.g. $[14,15]$. However, these are typically simulations where single ions or neutrals are treated, but the effect of a plasma and of its nonequilibrium properties [cf. Fig. 2] and the plasmainduced modifications of the surface have not been taken into account so far.

From the examples presented above that demonstrated the mutual influences between plasma and surface, it is clear that further progress in an accurate modeling of the plasma-solid interface requires to go beyond an independent treatment of both sides. Instead it is necessary to develop a combined theory and simulation of the entire system. This is a challenging project that requires strong input from plasma physics and surface science, simultaneously. In fact, such a research effort is under way at Kiel University, in collaboration with Greifswald University and the INP Greifswald. The concept of this project was first presented in 2015 [16] and continuously developed since then. It is the goal of this article to present these ideas and first simulation results and to outline further directions and perspectives of development. We note that similar concepts have been developed by Graves, Brault and Neyts and others in the frame of MD simulations, e.g. [17,18], see Sec. IV. The main difference is that those simulations usually neglect the electronic degrees of freedom, in particular, quantum effects and internal relaxation processes in the solid.

This paper is organized as follows. In Sec. II we discuss the mutual influences between plasma and solid that motivates the development of a novel approach combining plasma and surface science methods. In Sec. III we discuss more in detail the relevant physical processes and effects at the plasmasolid interface. This sets the basis for the required theoretical approaches that are capable to accurately simulating plasmasurface processes and discuss their respective advantages and problems. We identify four different methods that are discussed in detail with increasing degree of complexity. In Sec. IV we discuss the first one - mesoscopic approaches to the plasma-solid interface - in particular molecular dynamics and discuss acceleration approaches that are of potential relevance for plasma-surface interaction. In Sec. V we discuss the second class of methods that is based on the quantum Boltzmann equation. In Secs. VI we consider the third approach that is based DFT and TDDFT. Finally, in Sec. VII the fourth approach is discussed that is based on nonequilibrium Green functions (NEGF) and leads to generalized quantum kinetic equations. The analysis concludes with Sec. IX where we outline first steps towards an integrated plasma-surface modeling, and we present our conclusions in Sec. X.
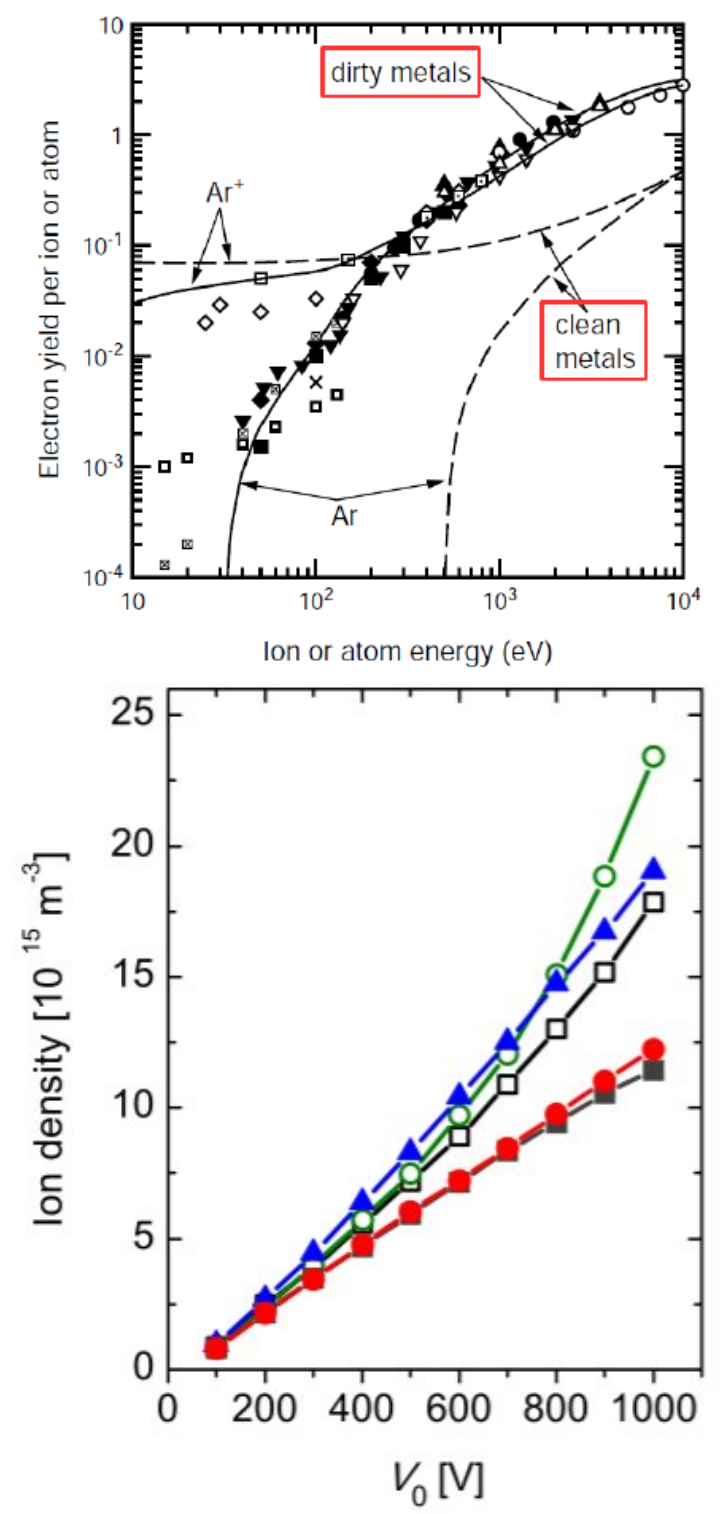

Figure 4: Top: Secondary electron emission yield per argon atom or ion for a broad variety of metals (symbols). "Clean metals" refers to beam experiments where the surface was cleaned via ion sputtering. "Dirty metals" denotes measurements following varying degrees of surface exposure to oxygen, to water or ambient gas. Figure from Ref. 12 where additional details are given. Bottom: Effect of SEE on ion density in the bulk of a AC discharge $(13.56 \mathrm{MHz}$, $\mathrm{p}=5 \mathrm{~Pa}$, for varying electrode voltage), for different SEE models: no SEE (black) to a full treatment of ion and atom induced SEE (green). From Ref. 11.

\section{MUTUAL INFLUENCES OF PLASMA AND SOLID}

As we discussed in the Introduction there are many processes that couple the plasma and the surface. Here we discuss a few examples. The first is the effect of energetic neutrals impacting the surface. These neutrals are efficiently produced in the case of a strong sheath electric field, by means of charge 
exchange collisions. Energetic neutrals maybe crucial for secondary electron emission from metal surfaces, as was shown in Ref. 12, see also Fig. 4. This was confirmed by PIC simulations where neutrals above a threshold energy of $23 \mathrm{eV}$ were traced individually [11].

An example where the surface properties affect the plasma is related to plasma electrons hitting a solid surface. The standard assumption in plasma simulations is that these electrons are lost without reflection, e.g. [19]. Only recently a microscopic calculation of the electron sticking coefficient was performed by Bronold and Fehske [20] that demonstrated that this picture has to be revised. Recent PIC simulations by Sun et al. confirmed that finite electron reflectivity, in combination with SEE, may have a significant influence on the plasma parameters of a CCRF discharge for pressures of several tens of $\mathrm{Pa}[21]$.

An even more striking example of the effect of the properties of the surface on the plasma properties is secondary electron emission. Phelps and Petrovic compiled extensive experimental data for the SEE yield from different metals over a broad range of impact energies of argon atoms and ions [12]. They compared SEE from surfaces that were cleaned by ion sputtering (cf. curves labeled "clean metals" in the top part of Fig. 4) to the SEE yield obtained from surfaces that have been in contact with a plasma (or to oxygen or ambient gas, for the original references see Ref. 12). The authors note a dramatic difference of the SEE yield from a "dirty" surface compared to a "clean" one which may exceed two orders of magnitude for SEE due to neutral atoms. For energetic atoms (energies above $200 \mathrm{eV}$ ) this difference is much larger than the difference between different metals.

The crucial importance of SEE has been confirmed in many simulations. For example, Derszi et al. performed PIC simulations where they included SEE according to various models via modified cross sections [11]. In that work it was found that a realistic ("dirty" [12]) surface gives rise to a significant increase (up to a factor of two) of the ion density, even far away from the electrode compared to simulations where SEE is neglected. This trend is seen in the bottom part of Fig. 4, compare the green and black curves. At the same time, the experimental data shown in the top part of Fig. 4 suggest that there remain substantial uncertainties in the values of the SEE coefficient. It is also not clear how long the surface was treated. In a real plasma treatment experiment a "clean" surface may correspond to the initial state of an electrode which, ultimately, turns into a "dirty" metal that is covered by adsorbates or an oxide layer. Thus, more accurate experimental and theoretical knowledge of the SEE for different materials and varying degrees of surface coverage will be very important for applications.

Strong surface effects have also been observed in the field emission. For example, Li et al. studied the effect of surface roughness on the field emission by including a phenomenological geometric enhancement factor [22].

These examples show that the simple concept of fixed phenomenological surface parameters, such as the SEE coefficient $\gamma_{e}$, for a given surface material, that was sketched in Fig. 3 has to be questioned. Instead the SEE coefficient has to take into account the properties of the surface and also the exposure to the plasma. In order to make reliable predictions,

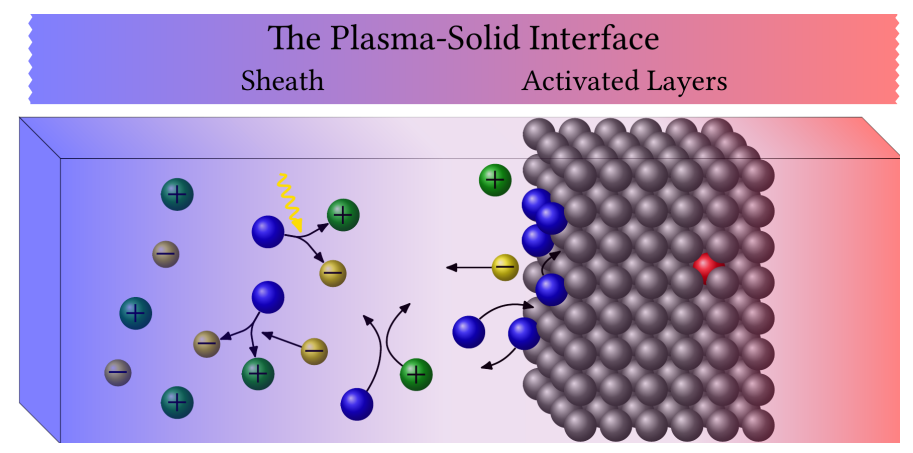

Figure 5: Sketch of the plasma-solid interface which comprises the plasma sheath and the plasma facing activated layers of the solid [16]. Among the relevant processes are diffusion, adsorption ("sticking") and desorption of neutrals, penetration (stopping) of ions and electron transfer between solid and plasma. The influence of the plasma on the solid and vice versa is a major challenge for a predictive theoretical treatment and require a combination of various theoretical approaches, see Fig. 7.

novel theoretical concepts are needed that include the whole scope of complex physical and chemical processes that occur at the plasma-solid interface which include secondary electron emission, sputtering, neutralization and stopping of ions, adsorption and desorption of neutral particles as well as chemical reactions. Therefore, the plasma-solid system should be treated as single entity which we call "plasma-solid interface" [16]. It comprises the plasma sheath and the plasma facing atomic layers of the surface that are influenced (excited or "activated") by the plasma. This new theoretical concept is sketched in Fig. 5.

\section{THE PLASMA-SOLID INTERFACE: PHYSICAL PROCESSES AND THEORETICAL APPROACHES}

\section{A. Overview on the physical processes at the plasma-solid interface}

Let us now look more in detail at the properties of the plasma-solid interface and the relevant processes. We already discussed in the beginning, cf. Fig. 1, that plasma and solid are characterized by a huge density gap leading, in many cases, to an enormous difference in length and time scales. First of all, the plasma-solid system is in a stationary state. This state differs from thermal equilibrium due to the nonequilibrium character of the plasma [cf. Fig. 2] and due to fluxes of particles (electrons, ions, neutrals) that cross the boundary in both directions.

On the largest scale (the scale of the Debye length, Fig. 6.a) the interface is characterized by the density profiles of electrons and ions. Electron depletion in the plasma sheath near a surface [cf. Fig. 3] gives rise to an excess positive charge in front of the surface. The missing negative charge has 
to accumulate inside the surface giving rise to an electric double layer. Charged double layers are a common phenomenon in liquids and were originally studied in electrolytes by Helmholtz [23]. The corresponding effect for the plasmasolid interface was predicted by Bronold and co-workers [24] and turns out to be very different, by the different composition of the system, and more complex, due to its nonequilibrium nature. The peculiarities of the electric double layer that are caused by the plasma properties-the non-Maxwellian velocity distributions and the time variation, in case of an rf field, on the scale of nanoseconds-are qualitatively understood. On the other hand, what is far less understood, is the impact of the solid on this charge distribution: the influence of the nanoscale surface structure [Fig. 3.b] as well as of the atomic scale structure [Fig. 3.c] and processes between solid and plasma.

Thus, an analysis of the surface and near-surface structure of the solid in the presence of the plasma and of surface processes under these conditions is required. This includes the structural and chemical response of the material. Surface science experiments are required to investigate the atomic-scale structure [Fig. 3.c], e.g., surface relaxation and reconstruction, adsorbate species, and surface defects, as well as the evolution of the nanoscale morphology, e.g., the formation of islands, pits, steps, and ultrathin films on the surface [Fig. 3.b]. Also the modification of the material in the near-surface region (e.g., crystallinity, porosity, composition, defect density) has to be studied as function, e.g., of ion and neutral particle energy and plasma density. It would be highly desirable that surface science and plasma physics experiments obtain the concentration of charged and neutral species at and near the surface as well as chemical binding energies of various species. The information that should be obtained from theory includes the relevant cross sections and rate coefficients, neutral sticking coefficients, SEE coefficients, diffusion coefficients, information on energy dissipation channels and time scales etc.

Another key topic is the charge transfer dynamics across the interface in the presence of the plasma. Here, plasma physics experiments are needed that provide key information, including the electron and ion fluxes to and from the surface [Fig. 3.a]. This should be complemented by surface science experiments measuring the secondary electron current from the surface and the plasma-induced modification of the band structure of the surface material. These quantities are the combined result of a multitude of physical processes. Theory and simulations should attempt to resolve the individual contributions due to the neutralization of ions in front of the surface or electron impact ionization and ion stopping inside the solid. Furthermore, it would be desirable if surface science experiments could provide insight into the complex energy landscape of the solid surface, its modification due to the plasma environment, as well as the plasma-induced space charge region inside the solid. The experimental information should be complemented by novel theoretical approaches that will lead to accurate results for electron and ion velocity distributions in the plasma sheath, on secondary electron emission, on the sticking coefficients of electrons, ions, and neutrals and on the charge of nanoparticles embedded in the plasma.
All these processes evolve in time (on the scale of seconds), as a result of the surface modification by the plasma. Thus, there is a direct coupling between effects on the atomic scale and the macroscopic plasma behavior that needs to be explored.

\section{B. Theoretical approaches for the plasma-solid interface}

Let us discuss, in the following the theoretical strategy needed to tackle these problems. Aside from the different pressure, length and time scales, the main difficulty is that both sides of the plasma-solid interface are governed by completely different physics: low-density gas-like behavior, in the plasma, versus quantum dynamics of electrons, in the solid, coupled to the lattice dynamics; this situation, the relevant processes and scales are sketched in Fig.7.

An accurate simulation of plasma-surface processes, first of all, requires a reliable description of the plasma and the solid. Standard tools in plasma simulations are fluid simulations and kinetic theory (cf. blue box in Fig. 7). Here two main approaches are in use: direct solution of the Boltzmann equation or particle in cell simulations with Monte Carlo collisions (PIC-MCC). The particle dynamics have to be coupled to the dynamics of the electromagnetic field on the basis of Maxwell's equations or the Poisson equation for the electrostatic potential. Finally, these simulations require surface parameters as an input- the fluxes $\mathbf{J}^{s}$ - and deliver the corresponding fluxes $\mathbf{J}^{p}$, as an output, cf. Fig. 7.

To obtain the necessary surface information, one first of all needs to obtain the ground state properties of the solid-the energy spectrum (band structure) and the Kohn-Sham orbitalswhich is done by density functional theory (DFT) simulations [Sec. VI], cf. right part of Fig. 7. However, DFT is known to have problems, in particular, in treating materials with strong electronic correlations including various oxides. Here, many-body approaches are being used that include the BetheSalpeter equation (BSE), e.g. [25], dynamical mean field theory (DMFT), e.g. [26], or quantum Monte Carlo (QMC) for the ground state or finite temperature [27,28].

Next, if the solid comes in contact with a low-temperature plasma, energetic electrons, ions or neutrals may excite the electrons of the solid and the lattice. This is already not captured by ground state DFT but requires time-dependent extensions, cf. the approaches listed in the central box of Fig. 7. The top row in the central box lists mesoscopic approachesmolecular dynamics (MD) and kinetic Monte Carlo (KMC)that do not treat the quantum dynamics of the electrons explicitly. This simplification allows one to access comparatively large time and length scales, see also Fig. 8. In particular with $\mathrm{KMC}$, in principle, one can reach minutes of simulation time and length scales of centimeters. KMC has been successfully used in many plasma simulations, e.g. [30-34], and it is an integral part of many multiscale simulation concepts because it is very flexible with respect to the inclusion of new processes. However, all these processes are treated in a very simplified manner using process rates that often do not include all relevant parameter dependencies. In cases where the complete set 


Electric double layer

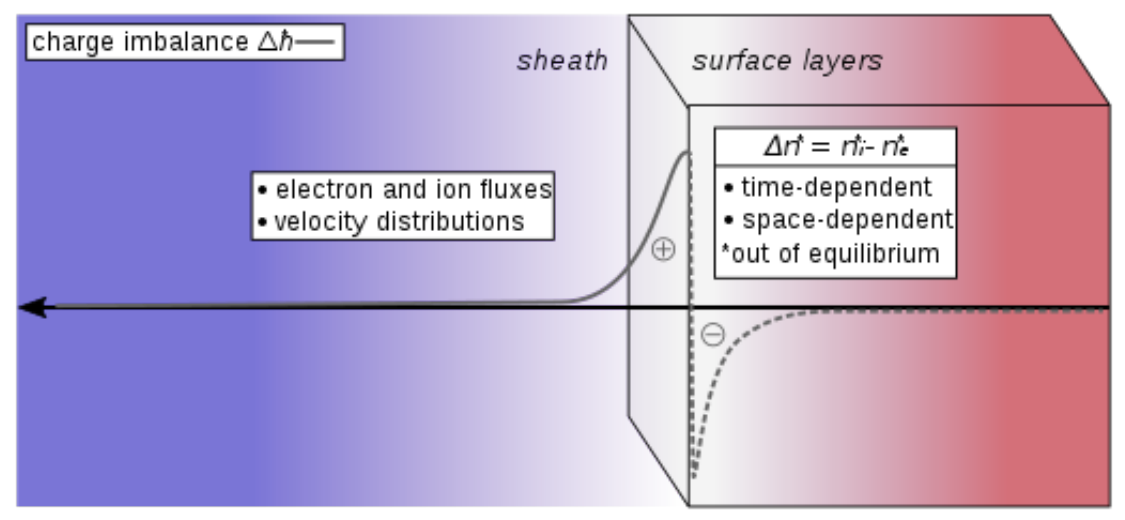

\section{\begin{tabular}{|ll}
\hline$b$ & Nanoscale morphology $\quad$ 回 Atomic interface structure and dynamics \\
\hline
\end{tabular}}

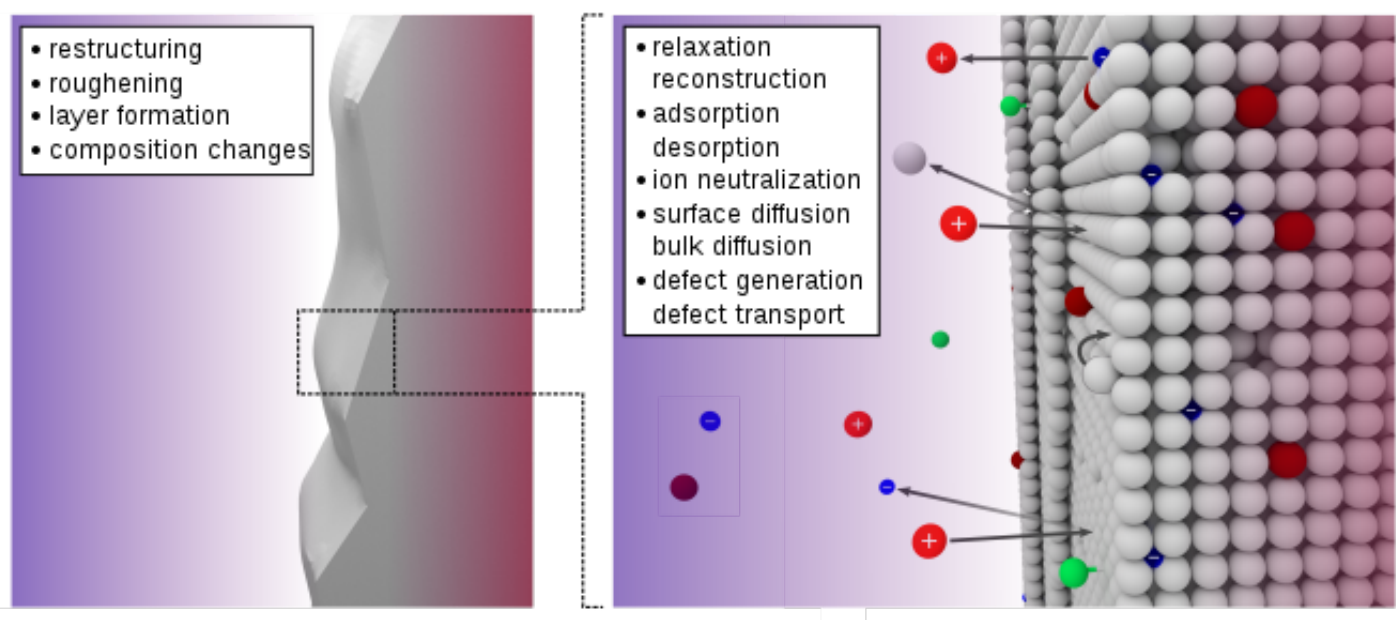

Figure 6: Sketch of the physical processes at the plasma-solid interface-from the largest to the smallest length scale. Top: The electric double layer (on the scale of the Debye length, on the plasma side, and a few nanometers, in the solid) resulting from electron depletion in the plasma sheath [cf. Fig. 3] is characterized by the local difference of the nonequilibrium ion and electron densities and is accompanied by electron accumulation in the solid which is influenced by the processes in figure parts b) and c). Bottom left: on the scale of the surface roughness (typically nanometers) the surface exhibits local variations of the morphology and chemical composition. Bottom right: atomic scale modification of the surface and the plasma sheath caused by individual particle impacts, charge transfer, chemical reactions etc. The relevant processes are indicated inside the figure parts. 


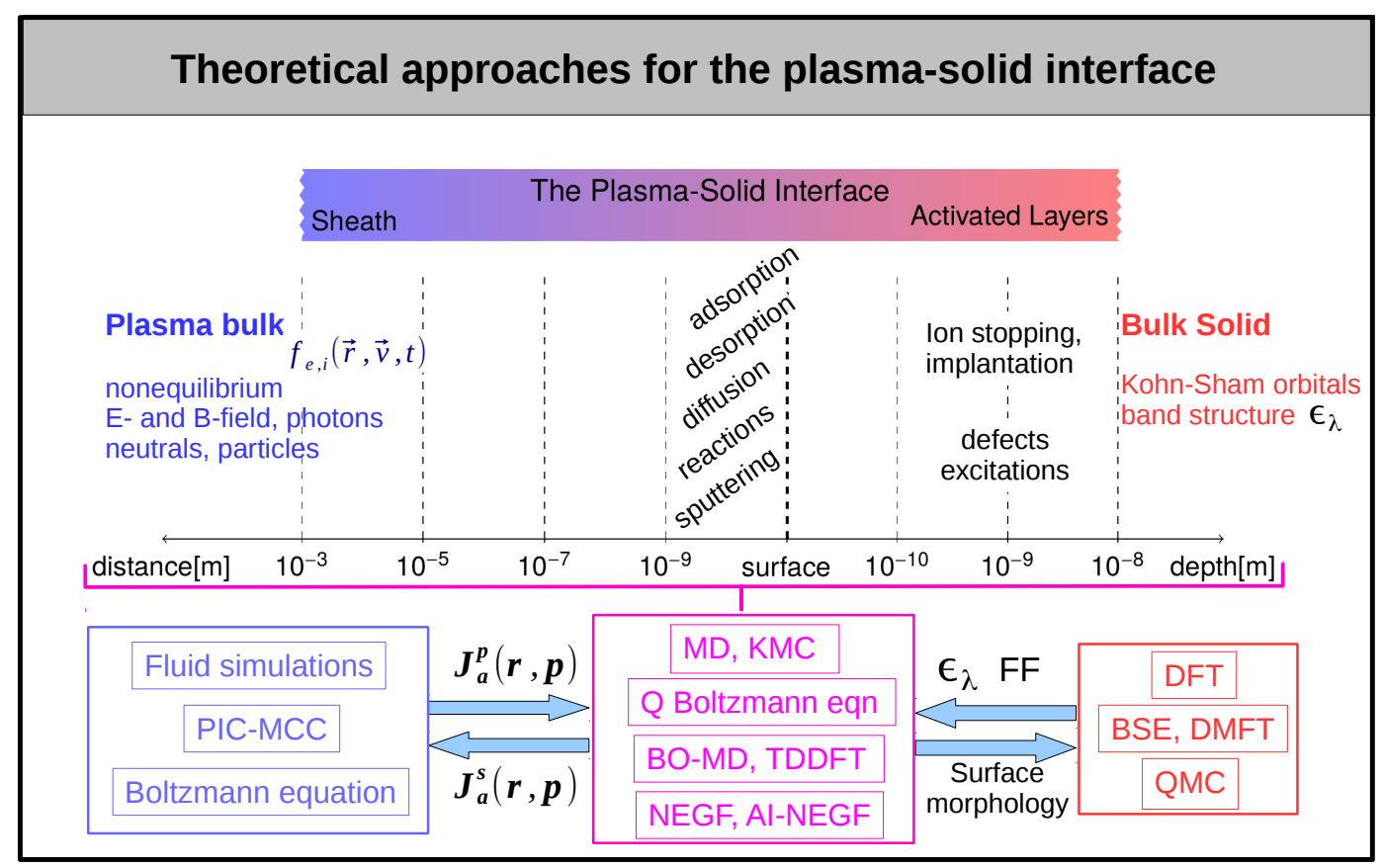

Figure 7: Theoretical methods for the description of the plasma-solid interface ${ }^{16}$, as sketched in Fig. 5. Some of the processes of interest are listed in the figure. Note the dramatically different length scales and the very different properties of the plasma and the solid, requiring fundamentally different methods to be applied on the plasma and the solid side. Standard methods for the bulk solid are Density functional theory (DFT), Bethe-Salpeter equation (BSE), Dynamical Mean Field Theory (DMFT), and Quantum Monte Carlo (QMC). To simulate surface processes (central box), additional non-adiabatic (time-dependent) approaches are required: molecular dynamics (MD), Kinetic Monte Carlo (KMC), Quantum Boltzmann eqution, Born-Oppenheimer MD (BO-MD), time-dependent DFT (TDDFT), Nonequilibrium Green functions (NEGF) and ab initio NEGF (AI-NEGF). To account for the complex interactions between plasma and solid, the corresponding methods have to be properly linked: plasma simulations should provide the momentum dependent fluxes $\mathbf{J}_{a}^{p}$ of all species "a" to the surface whereas surface simulations deliver the corresponding fluxes $\mathbf{J}_{a}^{s}$ that leave the surface. Bulk solid simulations provide the band structure $\epsilon_{\lambda}$ and reactive force fields (FF), whereas surface simulations return the updated surface morphology, chemical modifications etc. For details see text.

of relevant processes is well known, KMC may, nevertheless, be a powerful tool. In contrast, for new problems-such as the plasma-solid interface-where the complete set of events is not known a priori, the accuracy and predictive power of $\mathrm{KMC}$ is rather limited. We will, therefore, not discuss KMC in detail here-the interested reader is referred to Refs. 29-31,35. Instead, here we will concentrate on MD because it has a much stronger foundation and can retain a first-principle character once the information on the interaction potentials is derived from $a b$ initio approaches in cases when electronic and quantum effects are not important. We will give a more detailed discussion in Sec. IV. For an overview on the length and time scales that can be reached by MD and the other methods, see Fig. 8.

The second row in the central box of Fig. 7 lists models based on the quantum Boltzmann equation - the quantum generalization of classical kinetic equations. Here extensive recent work is due to Bronold and co-workers who developed simulations of the charge transfer, electron sticking and other processes. The corresponding approach and some results will be summarized in Sec. V.

The third row in the central box of Fig. 7 is devoted to timedependent simulations that are based on DFT. The first approach is Born-Oppenheimer MD where the ions are moved with classical molecular dynamics whereas the electrons are assumed to follow the ion dynamics adiabatically, thereby remaining in the (time evolving) ground state that is obtained quantum-mechanically, by ground state DFT. However, for strong excitation and/or fast processes the adiabatic approximation fails. Even though in plasma-surface interaction the mean excitation of the surface (per atom) may be small, local excitations may be strong, e.g. due to the impact of plasma particles. The corresponding non-adiabatic extension of DFT is time-dependent DFT (TDDFT) [36]. TDDFT is successfully being used for many surface processes. Applications to ion stopping have been performed e.g. in Ref. 14. An overview on this method is presented in Sec. VI.

Finally, the fourth row in the central box contains another $a b$ initio method: nonequilibrium Green functions (NEGF), e.g. [37,38]. This method generalizes the quantum Boltzmann equation (second row, Sec. V) to fast processes and is, in particular, well suited to accurately treat electronic correlation effects in the surface material. First applications to ion stopping were performed recently [15]. Finally, we also list $a b$ initio NEGF simulations - a recently developed combination of ground state DFT and NEGF [39]. An overview on NEGF methods will be presented in Sec. VII.

Even though the ab initio methods are, obviously, the most 


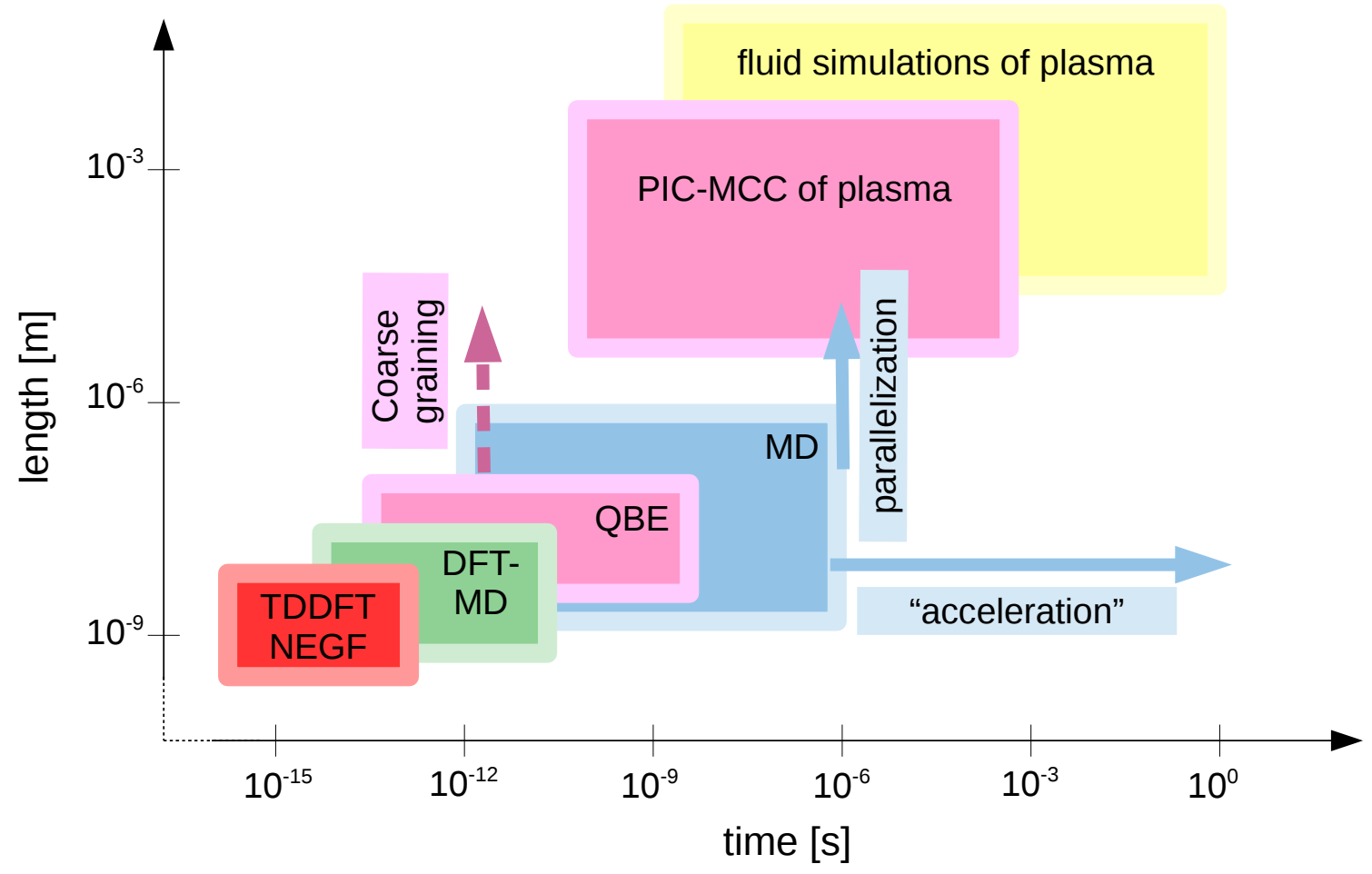

Figure 8: Approximate length and time scales accessible with different simulation methods for plasma-surface applications that are listed in Fig. 7. The shortcuts are the same as in Fig. 7 except for DFT-MD, which is equivalent to BO-MD, and QBE which stands for Quantum Boltzmann equation. Note that the comparison is only qualitative as different methods may apply to different processes. Also, the physical time resolution is often much less (larger minimum scale) than the required time step that is dictated by numerical stability. The $a b$ initio approaches TDDFT and NEGF resolve the electronic length and time scales and apply to ultrafast processes. DFT-MD has a more limited resolution of electronic relaxation processes. The upper limits of DFT, TDDFT and NEGF are set by the required basis dimension and accessible number of time steps. QBE resolves spatial details on the level of 100 inter-atomic distances and the relaxation time of the electrons in the solid. The upper length limit is determined by the imposed accuracy (level of coarse graining, pink arrow). MD propagates only the heavy particles neglecting electronic degrees of freedom. The accessible simulation dimensions can be increased via parallelization. Simulation times are restricted by the number of time steps and can, in some cases, be extended by additional "acceleration" methods (blue arrows). On the plasma side, PIC-MCC simulations resolve approximately one tenth of the electron Debye length and one plasma period and may extend to centimeters and milliseconds. Fluid simulations contain an additional coarse graining with respect to the particle velocities which limits their lower scale limits, compared to PIC, but extends their upper limits. KMC may, in principle, extend plasma-surface simulations to minutes and millimeters but is not considered here due to its largely uncontrolled character for the present applications, see text. Figure adapted from Ref. 29.

accurate theoretical approaches, they are extremely CPU-time demanding which strongly limits the accessible length and time scales. For example, Born-Oppenheimer MD simulation require a time step around $0.1 \ldots 1$ femtoseconds, which allows one to treat on the order $100 \ldots 1000$ atoms for $1 \ldots 100$ picoseconds, during a week of simulations on massively parallel hardware, e.g. Ref. 40. The demand for TDDFT and NEGF is several orders of magnitude larger. Typical length and time scales are summarized in Fig. 8. Thus, despite their accuracy, it is prohibitive to apply $a b$ initio methods to all problems of the plasma-solid interface. They should be applied to those processes where such an involved treatment is without an alternative, in particular, when important processes would be lost otherwise, e.g. via an averaging or coarse graining procedure.

Even though there is an impressive list of applications of all four plasma-surface simulation approaches, until now these have been developed essentially in isolation from each other. 
At the same time there is a high need for smart combinations of the different methods to cover the length and time scales of the plasma-solid interface at a sufficient accuracy and to capture all relevant physical and chemical processes. We hope that the present analysis of each of theses methods will highlight their strengths and weaknesses and stimulate such combinations.

\section{MESOSCOPIC SIMULATION APPROACHES: MOLECULAR DYNAMICS. ACCELERATION AND EXTENSION CONCEPTS}

We start with the method that extends to the largest time and length scales of those that are listed in the center of Fig. 7: molecular dynamics [for the discussion of KMC, see Sec. III]. This method is based on empirical interaction potentials and does not include quantum effects in the dynamics of the particles, in particular no explicit electronic effects. This approach has to be clearly distinguished from ab initio MD (or BornOppenheimer) MD where the electrons are time-propagated as well using density functional theory simulations (see Sec. VI). Nevertheless, such a semiclassical modeling is often sufficient for the treatment of the dynamics of neutral particles on a surface: diffusion, adsorption and desorption or many chemical reactions-a technique that is well developed in surface science and in theoretical chemistry, e.g. Ref. 41. Similarly, MD simulations are well established in low-temperature plasmas, e.g. to compute first principle structural properties of dust particles [42] or the diffusion coefficient in a strongly correlated magnetized plasma [43]. MD simulations are also actively used in plasma-surface simulations, e.g. [17,18], and recent applications include cluster growth [44] and sputtering [13].

The semiclassical MD simulations solve Newton's equations for all particles exactly. The quality of the results, obviously, crucially depends on the accuracy of the input data, most importantly, the effective pair potentials or force fields. These quantities are usually derived from microscopic quantum simulations or are adjusted to reproduce experimental data. Typically MD simulations for atoms require a time step of the order of $1 \mathrm{fs}$ and can, in principle, treat huge systems by using massively parallel hardware. For example, Ref. 45 reported simulations of a system containing $10^{11}$ atoms that reach times of the order of several milliseconds. However, this is presently only possible on the largest supercomputers or on dedicated hardware, e.g. [46]. At the same time, even though parallelization allows to reach larger system sizes, it does not help to extend the simulation duration.

Despite these impressive records, it is clear that in the near future MD simulations for plasma-surface processes will remain many orders of magnitude short of system sizes and length scales needed to compare with experiments. In plasma physics, these are minutes and (at least) micrometers, respectively. Therefore, additional strategies are needed. One way is of course the use of additional approximations leading to simplified models at the expense of accuracy and reliability. Here, we are concentrating on other methods that avoid simplifications of the equations of motion and to retain the first principles character of the MD simulations. The idea is to invoke additional information on the system properties that allow one to effectively accelerate the simulations and/or to extend them to larger scales without loosing accuracy.

There exists a variety of acceleration strategies including hyperdynamics [47], metadynamics [48] or temperature accelerated dynamics [49], for additional comments see Sec. VI. A more recent concept is collective variable driven hyperdynamics [50] that was reported to achieve, for some applications, speed-ups of about nine orders of magnitude. These methods have been successfully applied in surface physics and chemistry, and a more detailed discussion of these very diverse acceleration/extension developments, of their respective strengths and limitations was presented recently in Ref. 51.

The above methods are not easily applied to the heterogeneous plasma-solid system. Here recently a multi-scale simulation concept has been proposed that overcomes the lowdensity problem of the gas phase, for details see Ref. [13]. Another approach developed by two of the present authors [44,52] uses a selective acceleration of some relevant processes and, thereby, achieved speed-ups exceeding a factor $10^{9}$, see Sec. IV A. The third direction of developments we underline here does not aim at accelerating the ab initio simulations but to extend them to longer times by a suitable combination with analytical models [53,54]. These methods will be called below Dynamical freeze out of dominant modes (DFDM) and are briefly discussed in Sec. IV B

\section{A. MD simulations employing selective process acceleration} (SPA)

The authors of reference 44 considered, as an example for plasma-surface interaction, the deposition of gold atoms onto a polymer surface. The MD simulations tracked each individual atom, its diffusion on the surface, the emergence and growth of clusters and, eventually, the coalescence of the latter. The influence of the plasma environment was treated statistically by taking into account the impact of energetic ions that leads to the formation of surface defects that trap incoming atoms and prevent their diffusion. Varying the fraction of trapped atoms allows to mimick the flux of ions to the surface. In the MD simulations the isotropic Langevin equation of motion for all gold particles with the mass $m$ and spatial coordinates $\mathbf{r}=\left(\mathbf{r}_{1}, \mathbf{r}_{2}, \ldots\right)$ were solved:

$$
m \ddot{\mathbf{r}}=-\nabla U(\mathbf{r})-\frac{m}{t_{\mathrm{damp}}} \dot{\mathbf{r}}+\sqrt{\frac{2 m k_{\mathrm{B}} T}{t_{\mathrm{damp}}}} \mathbf{R}(t),
$$

where the potential $U$ describes the interaction between gold particles. For this potential $a b$ initio force field data are being used (the MD simulations used the LAMMPS package). Further, $t_{\text {damp }}$ has the role of a damping parameter, and $\mathbf{R}$ is a delta-correlated Gaussian random process. This random force and the viscous damping simulate the effect of the polymer on the heavy gold particles. This is motivated by the fact that the interaction between gold atoms by far exceeds the one between gold and polymer, so details of the latter are of minor 
importance. While the first term on the r.h.s. of Eq. (1) favors cluster formation (gold atoms settle in the minima of the total potential), the last two terms induce a diffusive motion with the diffusion coefficient

$$
D=\frac{1}{m} k_{\mathrm{B}} T t_{\mathrm{damp}}
$$

Thus, it is clear that the utilization of the Langevin dynamics allows one to control the speed of the surface diffusion and bulk by choosing a specific combination of the temperature $T$ and the damping parameter $t_{\text {damp }}$. Beyond that, it is possible to add a spatial (or directional) dependence to the diffusion coefficient if one lets the damping parameters depend on the position of the particle.

Based on the above considerations, Abraham et al. [44] developed a procedure to simulate the growth of nanogranular gold structures on a thin polymer film. By choosing appropriate ratios of the damping parameters, one can make sure that the atoms spend most of the time in the surface layer where they perform a random walk. The use of Langevin dynamics is restricted to the polymer surface whereas in the plasma, the dynamics of the gold atoms are purely microscopic. This allows one to add particles to the system by creating particles at the top of the simulation box and assigning them an initial velocity towards the substrate. Therefore, it is possible to perform the simulation with values of the deposition rates $J_{\text {sim }}$ and diffusion coefficients $D_{\text {sim }}$ that are much higher than the values in typical experiments.

In Ref. [44], it was argued that the simulations yield an adequate description of a real experimental deposition process if the ratio $J_{\operatorname{sim}} / D_{\operatorname{sim}}$ is equal to the ratio $J_{\exp } / D_{\exp }$ of the corresponding quantities in the experiment. The idea behind that is that - at least at the early stage of the deposition process - the growth should be essentially determined by the average distance an atom travels on the surface between successive depositions of atoms. Hence, the absolute time of the process is assumed to be irrelevant. The results presented in Ref. [44] were obtained with a time step of $1 \mathrm{fs}$, and a damping parameter for the diffusion in $x$ - and $y$-directions of $1 \mathrm{ps}$. The temperature and the deposition rate were set to match the conditions of the experimental results in Ref. 55 for the sputter deposition of gold on polystyrene. Using these parameters, the direct MD simulation time for the growth of a thin gold film is roughly $10^{9}$ times shorter than the corresponding time in the experiment. Or in other words, the duration of the MD simulations could be extended by nine orders of magnitude.

To verify the validity of such a dramatic shift of the time scales and to obtain the applicability limits, comprehensive tests of the method against experimental results were performed, see also Refs. 29,56 for a discussion. In particular, as one accelerates only selected processes, i.e., the deposition of atoms and the diffusion of atoms on the surface, one has to make sure that the neglect of other processes, e. g., the relaxation of a cluster structure, does not lead to artifacts in the simulation results. In Ref. 44, the method was tested by comparing several quantities describing the evolution of the gold film morphology with the results of time-resolved in situ grazing incidence $\mathrm{X}$-ray scattering (GISAXS) experiments of
Schwartzkopf et al. [55]. Indeed, many of the observed features could be reproduced by the simulations, for film thicknesses up to $3 \mathrm{~nm}$. This thickness corresponds to an impressive effective simulation time of $367 \mathrm{~s}$ which is directly suited for comparison with measurements.

The present approach of selective acceleration of dominant processes can be generalized to other systems as well. A recent application concerned the deposition and growth of bimetallic clusters on a polymer surface [52] where the acceleration allowed one to study the very slow process of demixing of the two metals. Applications of this approach to various plasma processes should be possible as well. In addition to the deposition of neutral atoms, the method also allows one to describe the impact of ions and the growth of charged clusters.

\section{B. Dynamical freeze out of dominant modes (DFDM)}

We now discuss an approach that does not accelarate MD, as the ones mentioned above, but takes advantage of the intrinsic hierarchy of relaxation processes existing in any manybody system. When a system is excited, typically, small scale processes and correlation effects will tend to equilibrate fast whereas large scale effects such as particle transport will occur on longer time scales. In between these scales one expects the establishment of equilibrium (or stationary) velocity distributions, for details and more examples, see Refs. [51,57]. This means that during the course of the evolution the information and detail required to describe the entire process is systematically reduced. Finally, in thermodynamic equilibrium the system would be completely described by a few macroscopic variables such as temperature and density.

This hierarchical character of the evolution is known for a long time and also called "coarse graining". It means that a full microscopic description is only needed for the early period of the evolution whereas, for later times, it is sufficient to capture the dynamics of the relevant "modes" or degrees of freedom. This has lead to simplified models such as kinetic equations, fluid equations or rate equations where the full $\mathrm{N}$-particle information is mapped onto a limited number of quantities. Even though each of these equations is an approximation to the full many-body equations, these equations are accurate within their respective relaxation stages and time scales.

Of course, kinetic equations, fluid models or rate equations contain input parameters (such as collision cross sections or collision integrals, transport coefficients or reaction rates, respectively) that are usually derived by applying suitable approximations to the many-body problem. It is the accuracy of these approximations that governs the accuracy of the model. Consider, as an example, a master (or rate) equation

$$
\begin{array}{r}
\frac{d p_{i}(t)}{d t}=\sum_{j \neq i}\left\{\Gamma_{j \rightarrow i} p_{j}(t)-\Gamma_{i \rightarrow j} p_{i}(t)\right\}, \\
\quad 0 \leq p_{i}(t) \leq 1, \quad \sum_{i} p_{i}(t)=1,
\end{array}
$$

where $i$ is a multi-index numbering the configurations the sys- 
tem can have at some stage of the relaxation which occur with a probability $p_{i}(t) . \Gamma_{i \rightarrow j}$ are the transition rates (probability per unit time) from state $i$ to $j$. The first term on the right hand side of Eq. (3) describes processes which increase the probability to realize state $i$ ("gain"), whereas the second term describes the analogous loss processes.

Let us now return to our general question about the accuracy of the model (3). Such an equation can often be rigorously derived from the underlying classical or quantum equations of motion, and its accuracy is only limited by the accuracy of the transition rates $\Gamma_{i \rightarrow j}$ that are typically calculated using various approximation schemes. If, on the other hand, no approximations would be made and a general form be permitted where $\Gamma_{i \rightarrow j} \rightarrow \Gamma_{i \rightarrow j}\left(t ;\left\{p_{k}(t)\right\}\right)$ may depend on time as well as on all $p_{i}$ for the present and earlier times (memory) this would, in general, result in an exact equation (3).

In fact, in Ref. [51] it was proposed to obtain the exact expressions for $\Gamma_{a \rightarrow b}$ from first principle MD simulations. Imagine that in the course of the evolution the system reaches a state where only a small number of configurations can be realized the description would much simpler compare to the full N-body dynamics, even if the involved rates are complicated. Moreover, it can be expected that, in many cases, the functional form of the rates $\Gamma_{i \rightarrow j}\left(t ;\left\{p_{k}(t)\right\}\right)$ will simplify in the course of the evolution, and they even may converge to stationary values.

This procedure was demonstrated in Refs. [54,58] for a simple example: the adsorption kinetics of argon atoms on a platinum (111) surface. There it was shown that the sticking probability of the atoms can be derived from an equation of the type (3) with just three different states. The transition probabilities between these states were computed by first principle MD, and their time dependence was analyzed. It turned out that, in fact, these probabilities converge to constant values, $\Gamma_{i \rightarrow j}\left(t ;\left\{p_{k}(t)\right\}\right) \rightarrow \Gamma_{i \rightarrow j}^{\mathrm{EQ}}$, within approximately $t=t^{\mathrm{EQ}} \approx 20 \mathrm{ps}$, i.e. as soon as the adsorbate atoms have equilibrated with the surface. Thus, using the first-principle transition probabilities obtained from MD in the master equation (or rate equation), its solution will be essentially exact, for times $t \geq t^{\mathrm{EQ}}$. This allows one to extend first principletype simulations to times long enough to compare with experiments [51] without actually performing MD simulations. The reason is that one was able to identify the dominant collective modes that fully describe the system at long times and emerge dynamically during the evolution.

A similar approach was developed by Franke and Pehlke [53]. They performed DFT simulations of the diffusion of a 1,4-butanedithiol molecule on a gold surface and also mapped this on a master equation. Even though they did not consider in detail $a b$ initio results for the transition probabilities, their results confirm that the type of extension of accurate simulations can also be applied with quantum simulations, instead of semiclassical MD. The idea of mapping the microscopic dynamics onto a small set of relevant degrees of freedom is also utilized in the computation of the secondary electron emission coefficient. This also leads to a system of coupled rate equations as is explained in section $\mathrm{V}$.

\section{QUANTUM BOLTZMANN EQUATION}

In the previous section the focus was on semiclassical or even classical methods for describing the interaction of atoms with surfaces, based on Newton'a equations of motion for the constituents involved and their solution by molecular dynamics techniques. Not all surface scattering processes can be treated in that manner. In particular, charge-transferring processes require a quantum-mechanical approach. The most prominent scattering process (cf. Sec. II), which has to be treated quantum-mechanically and, at the same time, has great relevance for plasma modeling, is secondary electron emission from surfaces/plasma walls due to impacting heavy species.

\section{A. Quantum Kinetic Approach to secondary electron emission. Generalized Newns-Anderson model}

It has been known for a long time that secondary electron emission is an important process in bounded plasmas, affecting the structure of the plasma sheath, the overall charge balance, and the operation modii of basically all types of lowtemperature discharges [59]. To quantify the process is, however, a rather challenging task. Experimentally it requires sophisticated instrumentation and theoretically it asks for the solution of a scattering problem involving many-body targets and projectiles [60-62]. It is thus not surprising that little is known quantitatively about secondary electron emission from plasma-exposed surfaces which, moreover, are usually also insufficiently well characterized. The collection and discussion of secondary electron emission data by Petrović and Phelps [63] is still the main reference, cf. Sec. II. Only recently the plasma physics community initiated a number of new investigations devoted to the issue $[64,65]$.

In the following we focus on electron emission at low impact energies where the atomic projectile remains outside the surface and emission is driven by the transfer of internal potential energy from the projectile to the target. In principle there are two theoretical approaches to the problem. The first approach attempts to describe the processes from first principles, using density functional theory or quantum-chemical techniques. There exist various facets of this approach (see, for instance, the work of More and coworkers [67] and the monograph [62]) depending on how many electronic and lattice degrees of freedom are kept at an ab-initio level. Ideally, one keeps all. But this sophistication can be hardly maintained in a realistic description of plasma-surface interaction. The second approach, more modest in theoretical detail and to be followed in this subsection, is to keep only the most important degrees of freedom taking part in the scattering process and to construct effective models for them. Typically these are Anderson-Newns models [68-70] where the collision trajectory of a projectile is prescribed externally, leading to a time-dependent Hamiltonian for the participating electrons. The matrix elements of the Hamiltonian can be, of course, calculated from first principles but it is more in the spirit of an effective modeling to parametrize the matrix elements by 
(a)

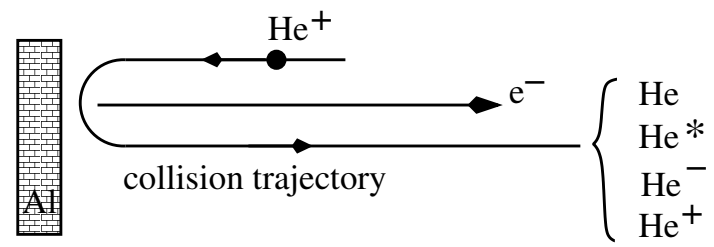

(b)

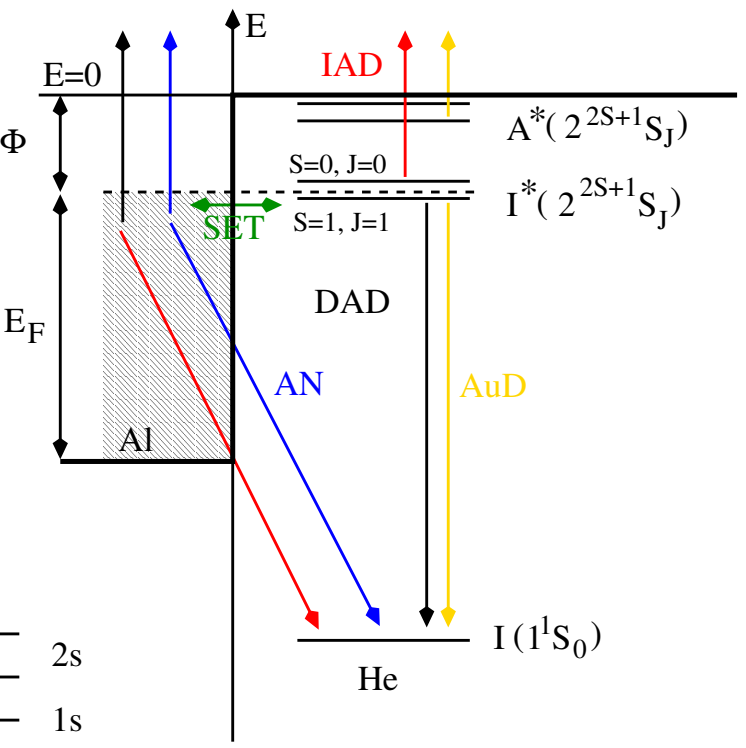

1011)

(c)

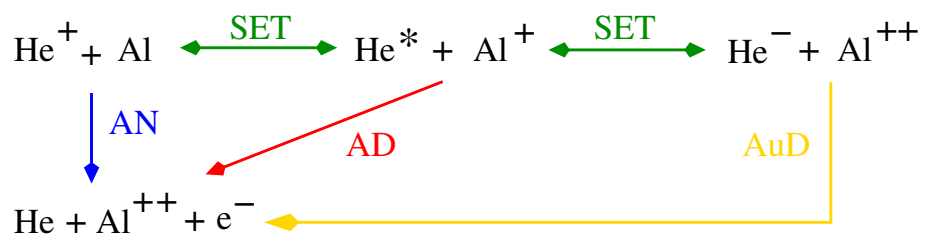

(d)

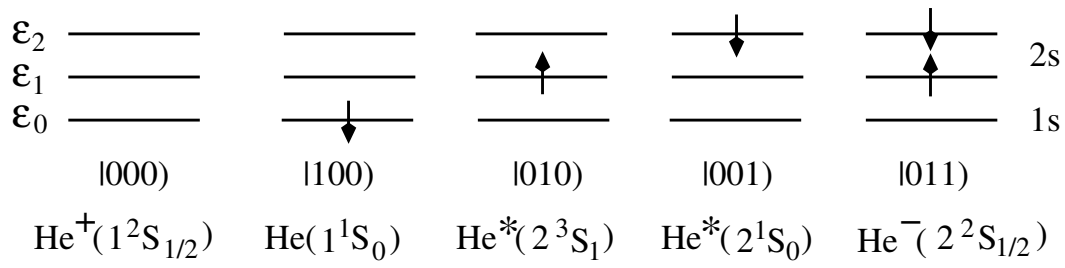

Figure 9: (Color online) Illustration of the main ingredients of an Anderson-News model based quantum-kinetic analysis of the neutralization of a helium ion on an aluminum surface characterized by a step potential with depth $E_{\mathrm{F}}+\Phi$, where $E_{\mathrm{F}}$ is the Fermi energy and $\Phi$ the work function of aluminum. The collision trajectory leading to time-dependent matrix elements enforcing a quantum-kinetic analysis is shown in (a) and the reaction channels included in the modeling are indicated in the (on scale) energy diagram (b) and the reaction diagram (c). For simplicity, the projectile levels shown are the ones far away from the surface, level shifts and broadening due to the interaction with the surface are not visualized. As indicated in (c), there are three routes to the projectile groundstate, each one leading to the emission of an electron. The helium ion may capture an electron from the metal by a single-electron transfer (SET), changing its configuration from $\mathrm{He}^{+}\left(1 \mathrm{~s}, 1^{2} \mathrm{~S}_{1 / 2}\right)$ to either $\mathrm{He}^{*}\left(1 \mathrm{~s} 2 \mathrm{~s}, 2^{3} \mathrm{~S}_{1}\right)$ or $\mathrm{He}^{*}\left(1 \mathrm{~s} 2 \mathrm{~s}, 2^{1} \mathrm{~S}_{0}\right)$, which may either Auger de-excite (AD) to $\mathrm{He}^{0}\left(1 \mathrm{~s}^{2}, 1^{1} \mathrm{~S}_{0}\right)$ or attract another electron from the metal to form a $\mathrm{He}^{*-}\left(1 \mathrm{~s} 2 \mathrm{~s}^{2}, 2^{2} \mathrm{~S}_{1 / 2}\right)$ ion releasing an electron then by auto-detachment (AuD). In addition to these two routes the $\mathrm{He}^{0}\left(1 \mathrm{~s}^{2}, 1^{1} \mathrm{~S}_{0}\right)$ configuration may be also reached by Auger neutralization (AN) of the $\mathrm{He}^{+}\left(1 \mathrm{~s}, 1^{2} \mathrm{~S}_{1 / 2}\right)$ ion setting also free an electron. Panel (d) finally depicts the three-level system which can be employed to represent the helium configurations taking part in the collision. Depending on the process the levels act as ionization or affinity levels. Auxiliary bosons are used to assign the functionality needed to the levels. Not included in the three level modeling, since it is unaffected by the collision, is the spin-up electron in the 1 s-shell common to all configurations listed in (d). For details see Ref. 66.

physical considerations. The resulting time-dependent Hamiltonian is then fed into a quantum-kinetic treatment, based on nonequilibrium Green functions (NEGF), which, if combined with pseudo-operator techniques, has the advantage that all collision channels, which may open-up when the projectile approaches the target surface, can be treated simultaneously. The NEGF approach is the same as listed in the fourth line of the pink box in Fig. 7 and will be discussed in some detail in Sec. VII. Here we will concentrate on the main steps that are involved in deriving the quantum Boltzmann equation from the NEGF equations and on the derivation of the rate equations model for the relevant degrees of freedom.

The use of Anderson-Newns models for the description of charge-transferring atom-surface collisions is well established and has a long history, recent applications [66,71-78] differ only in the way the matrix elements are obtained and the number of channels included. Both of course depend on the scattering process to be modelled. It is beyond the scope of this paper to review it here. Instead, we describe the Anderson-News type modeling developed at the Univer- sity Greifswald $[73,74,77,78]$ taking a helium ion hitting an aluminum surface as an example. The description is necessarily sparse, details and data for other metals can be found in the work of Pamperin, Bronold, and Fehske [66] to which we refer as PBF.

Figure 9 summarizes the approach as applied to the collision of a positive helium ion with an aluminum surface. In accordance with the general spirit of the Anderson-Newns model [68-70] the center-of-mass motion of the ion, for simplicity assumed to be normal to surface, is put on a prescribed trajectory. Due to the large mass of the projectile, the motion is indeed to a good approximation classical. It leads however to time-dependent single- and two-particle matrix elements and in turn to the necessity of using quantum kinetics to extract from the model the secondary electron emission coefficient $\gamma\left(\varepsilon_{\vec{q}}\right)$, where $\varepsilon_{\vec{q}}$ is the energy of the emitted electron. The electronic degrees of freedom of the projectile and the target are treated quantum mechanically, using a step potential of depth $E_{F}+\Phi$ to mimic the aluminum surface, with $E_{F}$ the Fermi energy and $\Phi$ the work function, and a few- 
level system to emulate the electronic states of the projectile affected by the collision, that is, the ionization and affinity levels which may take part in an electron transfer. As indicated, there will be typically more than one ionization and affinity levels involved. As a result, electron emission can occur via many channels. In the case depicted there are three main channels: Auger neutralization (AN), direct (DAD) and indirect (IAD) Auger de-excitation, and auto-detachment (AuD). All three can be included in the present NEGF approach and the quantum Boltzmann equation derived from it. It is sensible to parametrize the model Hamiltonian such that, far away from the surface, the energy levels of the projectile coincide with the experimental ionization energies and electron affinities of the isolated helium projectile. As it comes closer to the surface it starts to interact with it leading to level shifts and broadening (not shown in the figure), as well as to electron transfer due to Auger- and single-electron processes. For the results presented below the matrix elements of the model Hamiltonian were obtained from mean-field wave functions and physical considerations, taking image charges $[79,80]$ as well as tunneling through potential barriers [81,82] into account in case they arise. Details are given in PBF [66]. If instead of the physics-guided manner the matrix elements are obtained from first-principles, the quantum-kinetic treatment of the Hamiltonian described below remains the same.

The Hamiltonian describing the physics sketched in Fig. 9 is best written down in the notation of second quantization, that is, in terms of annihilation and creation operators [cf. Sec. VII]. Distinguishing between electron states belonging to the step potential, the few level system, and the unbound continuum, three types of Fermi operators are introduced: $c_{\vec{k} \sigma}^{(\dagger)}$, $c_{\vec{q} \sigma}^{(\dagger)}, c_{n}^{(\dagger)}$ annihilating (creating) an electron with spin $\sigma$ in, respectively, a surface state $|\vec{k}\rangle$, a continuum state $|\vec{q}\rangle$, and a projectile state $|n\rangle$. From a calculational point of view it is advantageous to replace the operators $c_{n}^{(\dagger)}$ by pseudo-operators, $e^{(\dagger)}, d^{(\dagger)}$, and $s_{n \sigma}^{(\dagger)}$ defined by

$$
\begin{aligned}
& |000\rangle=e^{\dagger}|\mathrm{vac}\rangle,|011\rangle=d^{\dagger}|\mathrm{vac}\rangle,|100\rangle=s_{1 \downarrow}^{\dagger}|\mathrm{vac}\rangle, \\
& |010\rangle=s_{2 \uparrow}^{\dagger}|\mathrm{vac}\rangle,|001\rangle=s_{2 \downarrow}^{\dagger}|\mathrm{vac}\rangle .
\end{aligned}
$$

They stand for whole electronic configurations of the projectile and not for single electron states. The number of electrons required for the electronic configurations represented by the pseudo-operators determines their statistics. Is the number odd (even) the operators obey Fermi (Bose) statistics. The labeling of the pseudo-operator is a reminder that the projectile's configurations involve the $1 \mathrm{~s}$ and $2 \mathrm{~s}$ shell of helium. In spectroscopic terms, the configurations included are the $\mathrm{He}^{+}\left(1 \mathrm{~s}, 1^{2} \mathrm{~S}_{1 / 2}\right)$ positive ion, the $\mathrm{He}^{0}\left(1 \mathrm{~s}^{2}, 1^{1} \mathrm{~S}_{0}\right)$ groundstate, the $\mathrm{He}^{*}\left(1 \mathrm{~s} 2 \mathrm{~s}, 2^{3} \mathrm{~S}_{1}\right)$ triplet and $\mathrm{He}^{*}\left(1 \mathrm{~s} 2 \mathrm{~s}, 2^{1} \mathrm{~S}_{0}\right)$ singlet metastable states, and the $\mathrm{He}^{*-}\left(1 \mathrm{~s} 2 \mathrm{~s}^{2}, 2^{2} \mathrm{~S}_{1 / 2}\right)$ negative ion. Employing the reasoning developed in $[74,77,78]$, the Hamiltonian describing the neutralization of a $\mathrm{He}^{+}\left(1 \mathrm{~s}, 1^{2} \mathrm{~S}_{1 / 2}\right)$ ion on an aluminum (or any other metal) surface within the scenario summarized in Fig. 9 becomes [66]

$$
\begin{aligned}
H(t) & =\varepsilon_{1 s \downarrow}^{0}(t) s_{1 \downarrow}^{\dagger} s_{1 \downarrow}+\sum_{\sigma} \varepsilon_{2 s \sigma}^{*}(t) s_{2 \sigma}^{\dagger} s_{2 \sigma}+\left[\varepsilon_{2 s \uparrow}^{-}(t)+\varepsilon_{2 s \downarrow}^{-}(t)\right] d^{\dagger} d+\sum_{\sigma} \omega_{\sigma}(t) b_{\sigma}^{\dagger} b_{\sigma}+\sum_{\vec{k} \sigma} \varepsilon_{\vec{k} \sigma} c_{\vec{k} \sigma}^{\dagger} c_{\vec{k} \sigma} \\
& +\sum_{\vec{q} \sigma} \varepsilon_{\vec{q} \sigma}(t) c_{\vec{q} \sigma}^{\dagger} c_{\vec{q} \sigma}+\sum_{\vec{k} \sigma}\left[V_{\vec{k} \sigma}^{\mathrm{SET}}(t) c_{\vec{k} \sigma}^{\dagger} e^{\dagger} s_{2 \sigma}+\text { H.c. }\right]-\sum_{\vec{k} \sigma}\left[\operatorname{sgn}(\sigma) V_{\vec{k} \sigma}^{\mathrm{SET}}(t) c_{\vec{k} \sigma}^{\dagger} b_{\sigma}^{\dagger} s_{2-\sigma}^{\dagger} d+\text { H.c. }\right] \\
& +\sum_{\vec{k}_{1} \vec{k}_{2} \vec{k}^{\prime} \sigma}\left[V_{\vec{k}_{1} \vec{k}_{2} \vec{k}^{\prime} \sigma}^{\mathrm{AN}}(t) c_{\vec{k}^{\prime} \sigma}^{\dagger} s_{1 \downarrow}^{\dagger} e c_{\vec{k}_{1 \downarrow}} c_{\vec{k}_{2} \sigma}+\text { H.c. }\right]+\sum_{\vec{k} \vec{k}^{\prime} \sigma}\left[V_{\vec{k} \vec{k}^{\prime} \sigma}^{\mathrm{DAD}}(t) c_{\vec{k}^{\prime} \sigma}^{\dagger} s_{1 \downarrow}^{\dagger} c_{\vec{k} \sigma} s_{2 \downarrow}+\text { H.c. }\right] \\
& +\sum_{\vec{k} \vec{q} \sigma}\left[V_{\vec{k} \vec{q} \sigma}^{\mathrm{IAD}}(t) c_{\vec{q} \sigma}^{\dagger} s_{1 \downarrow}^{\dagger} c_{\vec{k} \downarrow} s_{2 \sigma}+\text { H.c. }\right]+\sum_{\vec{q}}\left[V_{\vec{q}}^{\mathrm{AuD}} c_{\vec{q} \uparrow}^{\dagger} s_{1 \downarrow}^{\dagger} d+\text { H.c. }\right] .
\end{aligned}
$$

where we included auxiliary Bose operators $b_{\sigma}^{(\dagger)}$ enabling us to switch between projectile states with defects in their internal energy [66]. The physical meaning of the various terms is easy to grasp. For instance, the second last term stands for indirect Auger de-excitation (IAD) of the projectile (red arrows in Fig. 9b), that is, the creation of the groundstate of the projectile $\left(s_{1 \downarrow}^{\dagger}\right)$ by creating an electron in an unbound continuum state $\left(c_{\vec{q} \sigma}^{\dagger}\right)$ and annihilating either a singlet $\left(s_{2 \downarrow}\right)$ or a triplet $\left(s_{s \uparrow}\right)$ metastable state and an electron bound in the surface $\left(c_{\vec{k} \downarrow}^{\dagger}\right)$. The electron in the continuum is the secondary electron released in the course of Auger de-excitation.
The time-dependence of the Hamiltonian (5) reflects the dependence of most of its matrix elements on the actual position $z(t)$ of the projectile in front of the surface. The calculation of the matrix elements is analytically and numerically rather demanding. In fact, most of the computation time required for the quantum-kinetic modeling of secondary electron emission is allocated to the numerical evaluation of the matrix elements (and the selfenergies they give rise to). Explicit expressions for the matrix elements worked out along the lines developed in Refs. 74,77 are given in PBF [66]. The time-dependencies of the energies $\varepsilon_{1 s \downarrow}^{0}(t), \varepsilon_{2 s \sigma}^{*}(t), \varepsilon_{2 s \sigma}^{-}(t)$, and $\varepsilon_{\vec{q} \sigma}(t)$ are caused by long-range polarization effects and 
short-range non-orthogonality corrections. Assuming the projectile staying a few Bohr radii in front of the surface, the latter can be neglected while the former can be approximated by image shifts. The time-dependencies of the Coulomb matrix elements $V_{\vec{k}_{1} \vec{k}_{2} \vec{k}^{\prime} \downarrow}^{\mathrm{AN}}(t)$ for Auger neutralization, $V_{\vec{k} \vec{k} / \sigma}^{\mathrm{DAD}}(t)$ for direct Auger de-excitation, and $V_{\vec{k} \vec{q} \sigma}^{\mathrm{IAD}}(t)$ for indirect Auger de-excitation, as well as the time-dependence of the singleelectron transfer matrix element $V_{\vec{k} \sigma}^{\mathrm{SET}}(t)$ arise from the overlap of projectile and target wave functions which of course also depends on the separation $z(t)$ of the projectile and target.

\section{B. Derivation of the quantum Boltzmann equation for SEE}

Once the model is constructed it is analyzed within the quantum kinetic approach pioneered by Langreth and coworkers [83-85]. As result one obtains a linear set of or- dinary first order differential equations for the probabilities with which the projectile's electronic configurations occur in the course of the collision. In the case under discussion, one obtains equations for the occurrence probabilities of the ion, the groundstate, the two metastable states and the negative ion of the helium projectile. Three steps are required to obtain the rate equations. First, one has to set up two-time Dyson equations for the projectile Green functions $G$, cf. Eq. (11). Second, the selfenergies $\Sigma$ on the right hand side contain all interaction effects and have to be calculated. In the absence of strong electron-electron correlations on the projectile, the non-crossing approximation suffices for that purpose. Finally, the time integrals entering the selfenergies and the Dyson equations (11) are evaluated within a saddle-point approximation utilizing the fact that various functions are peaked around the time-diagonal. For more details, see Refs. 83-85 as well as Refs. 66,74,77,78. Following this reasoning one obtains the following system of rate equations

$$
\frac{d}{d t}\left(\begin{array}{l}
n_{+} \\
n_{\uparrow} \\
n_{\downarrow} \\
n_{-} \\
n_{g}
\end{array}\right)=\left(\begin{array}{ccccc}
-\left[\Gamma_{\uparrow}^{<}+\Gamma_{\downarrow}^{<}+\Gamma_{\mathrm{AN}}^{<}\right] & \Gamma_{\uparrow}^{>} & \Gamma_{\downarrow}^{>} & 0 & 0 \\
\Gamma_{\uparrow}^{<} & -\left[\Gamma_{\uparrow}^{>}+\Gamma_{-, \downarrow}^{<}+\Gamma_{\mathrm{IAD} \uparrow}^{<}\right] & 0 & \Gamma_{-, \downarrow}^{>} & 0 \\
\Gamma_{\downarrow}^{<} & 0 & -\left[\Gamma_{\downarrow}^{>}+\Gamma_{-, \uparrow}^{<}+\Gamma_{\mathrm{IAD} \downarrow}^{<}+\Gamma_{\mathrm{DAD} \downarrow}^{<}\right] & \Gamma_{-, \uparrow}^{>} & 0 \\
0 & \Gamma_{-, \downarrow}^{<} & \Gamma_{-, \uparrow}^{<} & -\left[\Gamma_{-, \uparrow}^{>}+\Gamma_{-, \downarrow}^{>}+\Gamma_{\mathrm{AuD}}^{<}\right] & 0 \\
\Gamma_{\mathrm{AN}}^{<} & \Gamma_{\mathrm{IAD} \uparrow}^{<} & \Gamma_{\mathrm{IAD} \downarrow}^{<}+\Gamma_{\mathrm{DAD} \downarrow}^{<} & \Gamma_{\mathrm{AuD}}^{<} & 0
\end{array}\right) \cdot\left(\begin{array}{l}
n_{+} \\
n_{\uparrow} \\
n_{\downarrow} \\
n_{-} \\
n_{g}
\end{array}\right)
$$

where $n_{+}(t), n_{\uparrow}(t), n_{\downarrow}(t), n_{-}(t)$, and $n_{g}(t)$ denote, respectively, the occurrence probabilities at time $t$ for the positive ion, the triplet and singlet metastable state, the negative ion, and the groundstate of the projectile. Expressions for the timedependent rates $\Gamma_{\ldots}^{\gtrless}(t)$, a discussion of the physical content of the rate equations as well as a route for obtaining the secondary electron emission coefficient $\gamma_{e}$ and the energy spectrum $\gamma_{e}\left(\varepsilon_{\vec{q}}\right)$ of the emitted electron from the solution of the rate equations are given in PBF [66].

To indicate the type of data which can be produced by the quantum-kinetic modeling of secondary electron emission, Fig. 10 shows numerical results for a positive helium ion hitting perpendicularly an aluminum surface with $E_{\text {kin }}=$ $60 \mathrm{eV}$ using the material parameters listed in PBF [66]. The left panel depicts the instantaneous occurrence probabilities $n_{+}(t), n_{-}(t), n_{\uparrow}(t), n_{\downarrow}(t)$, and $n_{g}(t)$ as well as the instantaneous probability $\gamma_{e}(t)$ for emitting a secondary electron. The energy spectrum of the emitted electron is plotted in the right panel. The projectile starts in the $\mathrm{He}^{+}\left(1 \mathrm{~s}, 1^{2} \mathrm{~S}_{1 / 2}\right)$ configuration at a distance $z=40 a_{\mathrm{B}}$, where $a_{\mathrm{B}}$ is the Bohr radius, moves along the trajectory shown in Fig. 9a towards the turning point $z_{\mathrm{tp}}=2.27 a_{\mathrm{B}}$, where it is specularly reflected to move back to the distance $z=40 a_{\mathrm{B}}$ to complete the collision. The probabilities for finding the projectile at the end of the collision in any one of the configurations included in the modeling can be read off from the left panel. For instance, the probability for returning in the groundstate configuration $\mathrm{He}^{0}\left(1 \mathrm{~s}^{2}, 1^{1} \mathrm{~S}_{0}\right)$ is $n_{g}\left(t_{\max }\right) \approx 0.96$ whereas the probability for surviving the collision in the $\mathrm{He}^{+}\left(1 \mathrm{~s}, 1^{2} \mathrm{~S}_{1 / 2}\right)$ configuration is $n_{+}\left(t_{\max }\right) \approx 0.04$. There is also a very small probability $n_{\uparrow}\left(t_{\max }\right) \approx 10^{-5}$ to come back in the $\operatorname{He}^{*}\left(1 \mathrm{~s} 2 \mathrm{~s}, 2^{3} \mathrm{~S}_{1}\right)$ triplet configuration. The probability for electron emission, the secondary electron emission coefficient $\gamma_{e}=\gamma_{e}\left(t_{\max }\right) \approx$ 0.07 . It has the correct order of magnitude suggesting that by careful testing, benchmarking, and comparison with experimental data the semi-empirical approach can be advanced to a level where it can produce very reliable data. The energy spectrum in the right panel indicates that the secondary electron can have rather high energies. It can thus become chemically very active in the gas discharge. A detailed analysis shows that for the aluminum surface secondary electron emission is dominated by Auger neutralization. For other metals, Auger de-excitation may become also important. The relative importance of the two channels depends on the line-up of the projectile's affinity and ionization levels with the Fermi level of the metal. It can thus be controlled by a judicious choice of the metal and the projectile, that is, in the plasma context, of the wall material and the composition of the background gas.

The presentation of the quantum Boltzmann equation approach for charge-transferring atom-surface collisions was couched in a particular application: secondary electron emission. It can be, however, also applied to other atom-surface 

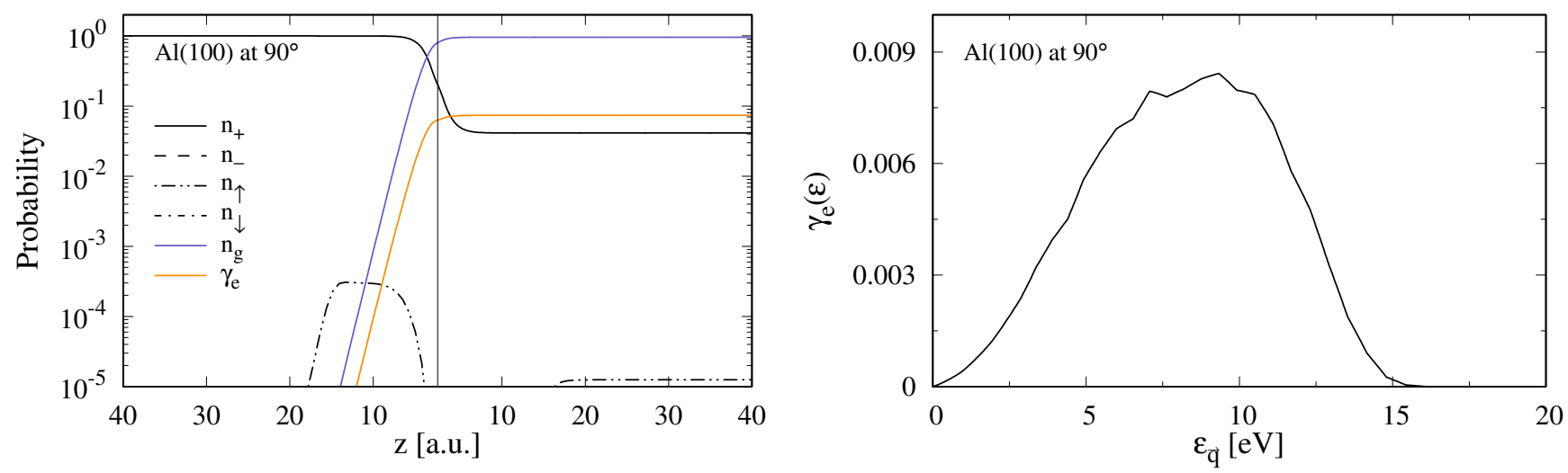

Figure 10: (Color online) Left panel: Instantaneous probabilities $n_{+}(t), n_{-}(t), n_{\uparrow}(t), n_{\downarrow}(t)$, and $n_{g}(t)$ for the projectile to be at time $t$ in the $\mathrm{He}^{+}\left(1 \mathrm{~s}, 1^{2} \mathrm{~S}_{1 / 2}\right), \mathrm{He}^{*-}\left(1 \mathrm{~s} 2 \mathrm{~s}^{2}, 2^{2} \mathrm{~S}_{1 / 2}\right), \mathrm{He}^{*}\left(1 \mathrm{~s} 2 \mathrm{~s}, 2^{3} \mathrm{~S}_{1}\right), \mathrm{He}^{*}\left(1 \mathrm{~s} 2 \mathrm{~s}, 2^{1} \mathrm{~S}_{0}\right)$, and the $\mathrm{He}^{0}\left(1 \mathrm{~s}^{2}, 1^{1} \mathrm{~S}_{0}\right)$ configuration together with the instantaneous probability $\gamma_{e}(t)$ for emitting an electron. The projectile hits the aluminum surface as a positive ion (solid black line) with a kinetic energy $E_{\text {kin }}=60 \mathrm{eV}$ and an angle of incidence with respect to the surface of $\varphi=90^{\circ}$. The thin vertical line denotes the turning point $z_{\mathrm{TP}}=2.27$, separating the incoming (left) form the outgoing (right) branch of the collision trajectory. The final probabilities, after the collision is completed, which are also the numbers relevant for plasma modeling, are the values at $z=40 a_{\mathrm{B}}$ on the outgoing branch. Right panel: Energy spectrum $\gamma_{e}\left(\varepsilon_{\vec{q}}\right)$ of the emitted electron after the collision is completed.

scattering processes affecting the electronic structure of the projectile-target system. The pseudo-particle representation of the projectile states opens the door for handling, within a single Hamiltonian, complex collisions, involving more than one channel. Numerically the approach is rather involved, preventing thus a quick production of surface parameters needed in plasma modeling. The main obstacle is the calculation of the matrix elements. So far they have been mostly obtained in a semi-empirical manner, guided by physical considerations and experimental data. Using $a b$ initio techniques, such as DFT [cf. Sec. VI], instead may eliminate some of the uncertainties of the matrix elements but the numerical effort will remain the same or even increase. Making the quantum Boltzmann equation-based modeling of charge-transferring atom-surface collisions numerically more efficient is one of the challenges for the future.

Finally, we notice that the quantum Boltzmann equation cannot resolve ultra-short time and length scales of the solid surface that are related electron correlations (cf. Fig. 8). The extension of quantum kinetic theory into these ranges can be achieved using the method of Nonequilibrium Green functions which is discussed in detail in Sec. VII. There we will also present a more accurate treatment of the solid surface that is complementary to the present approach.

\section{DFT-BASED $A B$ INITIO SIMULATIONS}

\section{A. Born-Oppenheimer MD}

Density functional theory (DFT) based methods constitute the most widely applied approach to calculate the electronic structure in physics and chemistry. It can be combined with Born-Oppenheimer Molecular Dynamics (BOMD) to simulate the dynamics of atoms within molecules and solids
[86]. Ab initio BOMD has been applied successfully by many groups, see e.g. Refs. 87-89. The BOMD approach starts from the Born-Oppenheimer (BO) approximation for the coupled system of nuclei and electrons [90], where all non-adiabatic coupling terms between the different $\mathrm{BO}$ surfaces of the electronically excited states are neglected. Furthermore, the classical limit for the motion of the nuclei is assumed, ignoring geometrical phases occurring around conical intersections [90]. The equation of motion (EOM) reduces to a set of coupled Newton's equations. Like in semi-classical MD described in Sect. IV, in case of an ensemble of $N$ atoms the micro-canonical MD simulation "boils down" to the integration of

$$
M_{i} \ddot{\mathbf{R}}_{i}=\mathbf{F}_{i}=-\nabla_{\mathbf{R}_{i}} U_{0}\left(\mathbf{R}_{1}, \ldots, \mathbf{R}_{N}\right)
$$

starting from some suitable initial conditions. $U_{0}\left(\mathbf{R}_{1}, \ldots, \mathbf{R}_{N}\right)$ denotes the ground state potential energy surface (PES) of the system. Canonical ensembles can be simulated by coupling to a Nosé-Hoover thermostat [91] or Nosé-Hoover chain thermostat [86]. Alternatively, the Langevin scheme can be used as detailed in Sect. IV.

As already pointed out, rare events pose a serious problem to any MD scheme, and even more so to the very CPU-time intensive $a b$ initio based MD programs. Thus, for the determination of reaction and diffusion energy barriers, algorithms like the nudged elastic band method (NEB, CI-NEB) $[92,93]$ are in use. Together with calculated vibrational frequencies, reaction and diffusion rates can be estimated within transition state theory (TST) [94]. The rates can be entered into a Master equation approach or kinetic Monte Carlo simulations as described in Sec.IV. In addition, Meta-dynamics approaches, as described by Laio and Parrinello [48,95] can help to escape local or global potential energy minima and explore further regions of the PES. A general difficulty when applying Metadynamics is the judicious choice of few collective variables for the simulation. 
While not without open basic problems, total energy calculations based on density functional theory (DFT) for the electronic ground state - together with a suitable approximation to the exchange-correlation energy functional - constitutes today's standard approach in electronic structure theory - both in physics and in chemistry [86,96-99]. This is in general ascribed to, in case of many applications, a favorable balance between the accuracy of the calculated total energy differences and forces acting on the ions as required for MD on the one hand and the (nonetheless still very large) computational costs on the other hand. DFT describes the exact mapping of the electronic ground state of the interacting many-particle Hamiltonian onto the ground state of a system of fictitious spin-1/2 particles not interacting with each other, which are moving in an effective potential that itself depends on the electron density $[100,101]$. The crucial point for practical applications is the required approximation to the exchangecorrelation (XC) energy functional. A careful choice of this approximation for the problem in question is essential for the accuracy of the obtained results. Various approximations are available. A universally applicable approximation to the XC energy functional giving chemical accuracy is lacking - this constitutes a field of active current research [97-99,102]. In many practical computations one of the generalized gradient approximations GGA-PW91 [103] or GGA-PBE [104] are applied. Other approximations comprise meta-GGAs [105], hybrid functionals, and many other approaches, see e.g. Refs. 97-99 for reviews. In view of MD simulations relevant for plasma physics of, e.g., noble gas atoms interacting with a metal surface, van der Waals forces (which cannot be represented by semi-local XC functionals like the local density approximation, LDA, or the GGAs) have to be considered $[54,102]$. Furthermore, as soon as open-shell atoms or other spin-polarized systems come into play, Spin-DFT has to be used [96].

Widely used DFT total-energy computer programs (e.g. VASP [106-109], the Quantum Espresso package [110,111] and various other programs) employ super-cells, which are repeated periodically in all three spatial directions, to simulate surfaces, clusters, etc., a plane-wave expansion of the Kohn-Sham orbitals and special k-points for approximate Brillouin zone integration [112]. The electron-ion interaction is represented by pseudopotentials [96] (e.g. norm-conserving [113-115], ultrasoft [116], projector augmented wave (PAW) $[117,118]$ pseudopotentials). In this way only the valence band states have to be computed, while the effect of the (frozen) core electrons is accounted for by the pseudopotentials. Again, special attention is required in case of MD simulations for plasma-surface interactions: If the kinetic energy of a projectile is so large that the inter-atomic separations become small during a collision, the pseudopotential approximation may become invalid and the interaction between the core electrons may have to be accounted for explicitly.

\section{B. Time-dependent DFT}

An ion from a plasma interacting with a solid surface [119124] results in inherently electronically non-adiabatic dynamics - which is beyond the realm of electronic ground state theory. Already the initial state, corresponding to an ion far away from the surface, is electronically strongly excited, although the individual parts of the system, i.e. the solid and the projectile, may initially be in their respective electronic ground state. Also the scattering of faster than thermal atoms at a metal surface can result in electronic energy dissipation, the description of which requires a simulation that accounts for electronically nonadiabatic effects (for an example see Ref. 125 for 2 - $10 \mathrm{eV} \mathrm{H}$ atoms impinging on an $\mathrm{Al}(111)$ surface). However, the deviation from the BO-surface is distinctly smaller in this case. In surface chemistry, electronically non-adiabatic effects have been observed experimentally (see., e.g. Refs. 126-129) and described theoretically by using various approaches including e.g. electronic friction [130-135], model Hamiltonians [136-140], or time-dependent density functional theory (TDDFT) [141-145]. Furthermore, also ion-atom collisions [146] and ions impinging onto a metal cluster [147,148], colliding with carbon nanostructures [149], graphene fragments [150], graphene or boron nitride (BN) [14,151], the collision of $\mathrm{Cl}, \mathrm{Cl}^{-}$with a $\mathrm{MoSe}_{2}$ monolayer [152] as well as the electronic stopping of atoms or ions moving through a solid [153157] have been simulated using TDDFT.

TDDFT [158-162] is based on the Runge-Gross theorem [163]. Under certain restrictions to the single particle potential, and given a fixed initial state $\left|\Psi\left(t_{0}\right)\right\rangle$, the electron density $n\left(\mathbf{r}^{\prime}, t^{\prime}\right), t^{\prime} \in\left[t_{0}, t\right]$ of a finite interacting many-particle system determines the time-dependent single particle potential $v(\mathbf{r}, t)+c(t)$ apart from an arbitrary function $c(t)$ of the time $[158,162]$. The interacting system can be mapped onto a system of fictitious spin-1/2-fermions with the same density not interacting with each other but moving in a time dependent effective Kohn-Sham potential [158,164]

$$
\begin{aligned}
v_{\mathrm{KS}}(\mathbf{r}, t)= & v(\mathbf{r}, t)+\int d^{3} \mathbf{r}^{\prime} \frac{n\left(\mathbf{r}^{\prime}, t\right)}{\left|\mathbf{r}-\mathbf{r}^{\prime}\right|}+ \\
& v_{\mathrm{XC}}\left([n],\left[\Phi_{0}\right],\left[\Psi_{0}\right]\right)(\mathbf{r}, t) .
\end{aligned}
$$

The XC-potential involves memory, it depends on the charge density history and the initial states $\Psi_{0}$ and $\Phi_{0}$ of the interacting and the Kohn-Sham system ${ }^{158,159}$. The crucial step, which is limiting the range of applicability of the respective approximate approach [165], is the approximation applied to the time-dependent XC-potential. Today, in most cases the adiabatic approximation is used (together with an approximation to the ground-state $\mathrm{XC}$ potential), i.e. the instantaneous electron density $n(\mathbf{r}, t)$ is inserted into an approximate local (e.g. LDA or GGA) XC-potential $v_{\mathrm{XC}}^{\text {approx }}$ from ground state DFT:

$$
v_{\mathrm{XC}}[n](\mathbf{r}, t) \approx v_{\mathrm{XC}}^{\text {approx }}[n(\cdot, t)](\mathbf{r}) .
$$

In the adiabatic approximation memory and initial state dependence are neglected. A critical discussion of these issues can be found in the article by N.T. Maitra [162] and 
the references cited therein and the article by Provorse and Isborn [165]. For the effect of different approximations to the ground-state XC-potential see e.g. Refs. 146,166. The effect of the approximate XC-potential e.g. on resonant charge transfer requires careful consideration, and comparison of time-dependent simulations of the many-body system within TDDFT-MD with results obtained from a manyparticle NEGF-approach, as described in Sec. VII, could provide additional insights to judge the accuracy. The application of time-dependent current density functional theory (TDCDFT) to stopping power and the effect of non-locality is discussed by V.U. Nazarov et al. in Ref. 167.

Another approximation is required for the description of the motion of the ions. The complete equations of motion for the combined system of nuclei and electrons can be written as a coupled system of equations, where the nuclei are moving on the ground and excited state Born Oppenheimer surfaces, depending on the electronic excitation [90]. Tully's surface hopping algorithm $[168,169]$ allows to simulate such time evolution stochastically. However, as long as the trajectories of the nuclei do not differ qualitatively but, instead, closely follow some average trajectory, the motion of the nuclei can be approximated by means of the much less expensive Ehrenfest dynamics [86],

$$
\begin{aligned}
M_{i} \frac{d^{2} \mathbf{R}_{i}}{d t^{2}}= & -\left\langle\nabla_{\mathbf{R}_{i}} \hat{V}_{\text {ion-el }}\left(\mathbf{R}_{1}, \ldots, \mathbf{R}_{n}\right)\right\rangle \\
& -\nabla_{\mathbf{R}_{i}} V_{\text {ion-ion }}\left(\mathbf{R}_{1}, \ldots, \mathbf{R}_{n}\right) .
\end{aligned}
$$

The expectation value in above equation has to be calculated with respect to the electronic wave function, which follows, in principle, from the integration of the time-dependent manyparticle Schrödinger equation of the electrons, or, in practical applications, the time-dependent Kohn-Sham equations. $M_{i}$ denotes the ion masses, $\mathbf{R}_{i}(t)$ the ion positions, and $V_{\text {ion-ion }}$ and $V_{\text {ion-el }}$ the ion-ion and ion-electron interaction potential energies.

Pseudopotentials [96] are in use in TDDFT-Ehrenfest MD simulations [170]. This enormously reduces the computational effort as compared to an all-electron approach [171]. Galilei invariance is preserved if non-local pseudopotentials are multiplied by an ion-velocity dependent gauge factor [166]. In case the projectile has some finite initial velocity, an initial boost has to be applied to electronic states of the projectile $[166,172]$ so that the electrons have the same initial velocity as the nuclei. The accuracy of TDDFT-MD simulations has been critically evaluated recently by Yost et al. [153] for the case of the electronic stopping power of a proton in Si. They report deviations between the all-electron and the pseudopotential approach and suggest a correction scheme. The differences arise at higher proton velocity (beyond 1 a.u.), and the correction is larger at small impact parameter [153]. A pseudoatom method to account for core electron effects has been applied to electronic stopping of e.g. $\mathrm{Li}^{+}$ions in graphene for impact energies beyond $10 \mathrm{keV} / \mathrm{u}$ in Ref. 151. As the deviation from the all-electron case will depend on the binding energy of the electronic states assumed as frozen core states in the pseudopotential construction, one should care about electron promotion effects missing in the pseudopotential calcu- lation [173] when carrying through TDDFT-MD simulations including high-energy head-on collisions.

TDDFT-Ehrenfest molecular dynamics is implemented, e.g., in the program octopus by Rubio et al. [170,174,175]. In their program, the Kohn-Sham wavefunctions are represented on a real space grid and systems of different dimensionality can be treated.

Applications of TDDFT-MD by many authors using different codes can be found in the literature. Examples have already been cited above, including electron stopping of $\mathrm{H}$ in Al, see e.g. Refs. $155,176,177$, the interaction of $\mathrm{Li}^{+}$with an $\mathrm{Al}$ cluster [147], the interaction of $\mathrm{H} / \mathrm{Al}$ in Ref. 125. The $\mathrm{H} / \mathrm{He}$ anomaly in the electronic stopping power in $\mathrm{Au}$ at low kinetic energies [178] and the role of the Au $d$ electrons has been simulated by the authors of Ref. 156. These applications refer either to electron stopping in bulk materials or, in case of the ion-discharge processes at surfaces, to resonant charge transfer, which is an inherently single-electron effect - as opposed to Auger neutralization, which is a two-electron effect [for a discussion, see Sec. V]).

It has been pointed out in the literature before that the energy transfer into electronic excitations can be simulated only for a finite time in case of a finite system due to its discrete energy spectrum $[123,179]$. Thus, e.g. in TDDFT-MD simulations of a vibrating molecule interacting with a metal surface $[144,180]$ the size of the unit cell (the number of atomic layers forming the metal slab or the cluster size) limits the simulation time. Within a simplified tight binding model for the case of a vibrationally excited $\mathrm{HCl}$ molecule incident on an $\mathrm{Al}(111)$ slab this has been exemplified by M. Grotemeyer [180]. A direct simulation of open quantum systems, see $e . g$. the article by R. D'Agosta and M. Di Ventra [181] and the article by $\mathrm{H}$. Appel in Ref. 159, would be advantageous.

For $\mathrm{Ar}^{8+}-\mathrm{Ar}$ charge transfer collisions Nagano et al. [166] observed that no Auger excited electrons occurred in their TDLDA-simulations. Furthermore, as shown by C.A. Ullrich for a model system in Ref. 182, when applying the ALDA for the XC potential in TDDFT doubly excited configurations are not accounted for. Based on the calculation of an exact XC-potential, V. Kapoor [183] has concluded that autoionization requires inclusion of memory effects in the XC-potential, which displays a rather complicated structure. When calculating e.g. the charge transfer between He ions scattered at an Al-surface, the two-particle character of the Auger transitions leading to the charge transfer between projectile and surface has to be accounted for, and other techniques are therefore used in case of Auger transitions [184,185].

To summarize, DFT and TDDFT simulation methods have undergone a very strong development since the formulation of the underlying theorems. We have discussed a number of practical applications and important approximations. However, we are not aware of direct applications to plasma-surface interaction yet. Of course, there have been many simulations of particular processes such as ion stopping, ion neutralization or neutral adsorption, but the influence of a nonequilibrium plasma environment is still an open question. DFT and TDDFT will, without doubt, play a key role in plasma-surface simulations. Their strength will be mostly in testing individ- 
ual processes, rather than simulating the full dynamics. This knowledge can then be used in other simulations such as those using quantum kinetic equations, cf. Secs. V and VII, e.g. as input for models describing the dynamics of electrons in ions, atoms or molecules (such as the Newns-Anderson model used in Sec. V), as well as for models of solids, such as lattice models used in Secs. VII and IX B.

\section{NONEQUILIBRIUM GREEN FUNCTIONS-BASED $A B$ INITIO SIMULATIONS}

We now return to the statistical approach to quantum manybody systems. We have already seen in Sec. V that a quantum generalization of kinetic theory allows for an efficient description of plasma-surface interaction processes such as secondary electron emission. The analysis of that section, however, used a simplified treatment of the solid, assuming spatial homogeneity and effective mass models and did not resolve the electronic dynamics in the material. Here we present a generalized quantum kinetic approach where these effects in the solid can be included straightforwardly. This will be demonstrated on the example of ion stopping focusing on recent simulation results of Balzer, Bonitz and co-workers $[15,186]$. At the same time, the NEGF approach is computationally extremely expensive and presently does not allow for a full quantum-mechanical treatment of the electron dynamics inside the projectile as well. So here an Ehrenfesttype dynamics will be employed, as was discussed already in Sec. VIB. This means that the present model is-in terms of effects included and neglected-complementary to both, the quantum Boltzmann approach presented in Sec. V and to the DFT concept of Sec. VI.

\section{A. Definitions and basic concept of NEGF}

The method of nonequilibrium (real-time) Green functions is a successfull approach to quantum many-body systems out of equilibrium, cf. Refs. 187,188. The method is a straightforward generalization of classical kinetic theory (e.g. Boltzmann equation) and of the quantum Boltzmann equation approach overcoming the limitations of the latter. These limitations include the restriction to times larger than the correlation time and fundamental problems such as incorrect conservation laws (e.g. conservation of kinetic energy instead of total energy) and relaxation toward an equilibrium state of an ideal gas (Fermi, Bose or Maxwell distribution) instead of the one of an interacting system, for a detailed discussion, see Refs. 57,189-191. Generalized quantum kinetic equations that are based on nonequilibrium Green functions (or, alternatively, on density operator theory [57,192]) overcome these problems.

The NEGF approach has been successfully applied to an impressively diverse array of systems, including nuclear matter, by Danielewicz, Köhler and others, e.g. Refs. 193,194, to optically excited semiconductors and quantum dots by Schäfer, Haug, Banyai, Bonitz and others, e.g. Refs. 190,195-
199, to dense laser plasmas by Kremp and Bonitz, e.g. Refs. 200,201, to few electron atoms, Refs. 202-204, and correlated fermions in lattice systems, Refs. 205,206 and many other problems. In the present context of plasma-surface interaction we expect that the NEGF approach will allow one to study non-adiabatic effects, in particular, relaxation processes in the surface that are initiated by the impact of plasma particles.

The NEGF-method is formulated in second quantization (for textbook or review discussions, see Refs. 38,188,202), in terms of creation (annihilation) operators $c_{i \sigma}\left(c_{i \sigma}^{\dagger}\right)$ for electrons in a single-particle orbital $|i\rangle$ with spin projection $\sigma$. Below we will consider a spatially inhomogeneous lattice configuration where $i$ labels the spatial coordinates of individual lattice points. The creation and annihilation operators are time-dependent via the Heisenberg representation of quantum mechanics. The central quantity that determines all time-dependent observables is the one-particle NEGF (we use $\hbar=1)$,

$$
G_{i j \sigma}\left(t, t^{\prime}\right)=-i\left\langle T_{\mathcal{C}} c_{i \sigma}(t) c_{j \sigma}^{\dagger}\left(t^{\prime}\right)\right\rangle,
$$

where the expectation value is computed with the equilibrium density operator of the system. For completeness we mention that times are running along the Keldysh contour $\mathcal{C}$, and $T_{\mathcal{C}}$ denotes ordering of operators on $\mathcal{C}$ (this is merely a formal trick for the theory development, all practical calculations are done for real-time quantitites, for details see Ref. 37). For example, the time-dependent electron density on site $i$ follows from $G$ via $n_{i}(t)=-i G_{i i \sigma}\left(t, t^{+}\right)$, where $t^{+} \equiv t+\epsilon$, with $\epsilon>0$ and $\epsilon \rightarrow 0$. If the site indices are taken different, $i \neq j$, the Green function describes time-dependent transitions of electrons between two lattice sites. In similar manner one computes the density matrix, currents, mean energies, optical absorption or electrical conductivity from $G$.

The NEGF obeys the two-time Keldysh-Kadanoff-Baym equations (KBE) [188]

$$
\begin{gathered}
\sum_{k}\left[i \partial_{t} \delta_{i k}-h_{i k \sigma}(t)\right] G_{k j \sigma}\left(t, t^{\prime}\right) \\
=\delta_{\mathcal{C}}\left(t-t^{\prime}\right) \delta_{i j}+\sum_{k} \int_{\mathcal{C}} d s \Sigma_{i k \sigma}(t, s) G_{k j \sigma}\left(s, t^{\prime}\right)
\end{gathered}
$$

where $h$ contains kinetic, potential and mean field energy contributions whereas correlation effects are included in the selfenergy $\Sigma$ [here we do not consider spin changes and we omit the second equation which is the adjoint of (11)].

Without the right hand side, Eq. (11) would be equivalent to a Vlasov equation or its quantum generalization (timedependent Hartree-Fock, TDHF). The r.h.s. contains correlation effects that are responsible for relaxation, dissipation and include scattering of electrons with electrons, ions or lattice vibrations (phonons). Notice the time integral on the r.h.s. which incorporates memory effects that are important to correctly treat correlations. The standard Boltzmann equation [Sec. V] is recovered by evaluating this time integral approximately via a retardation expansion $[57,207]$ or by a saddle point method as done in Sec. V. In this Section we will not consider this long-time limit but concentrate on the fast ion stopping dynamics in a solid. 
The NEGF formalism is formally exact if the selfenergy would be known exactly. The approach is internally consistent, obeys conservation laws and is applicable to arbitrary length and time scales. Its accuracy is determined by the proper choice for a single function - the selfenergy. For an overview on the treatment of weak and strong correlations in solids an optical lattices, see Ref. 38. In the following we consider, as an example of relevance to plasma-surface interaction, the NEGF approach to the energy loss of energetic ions in a solid.

\section{B. Nonequilibrium Green functions approach to ion stopping in nanomaterials}

The energy loss of energetic ions in a solid (stopping power) is an old problem that has been studied in great detail. There exist extensive references and successful code packages such as SRIM [208]. However, standard methods assume linear response, i.e. the material is weakly perturbed by the projectile and the response is computed in perturbation theory. While this maybe correct when considering an extended area of the surface where the effect of the projectile is small, on average, locally the excitation maybe strong. In the following we, therefore, attempt to perform a space and time resolved analysis of the projectile-solid interaction including electronic correlation effects in the solid. This will be particularly important for strongly correlated materials.

We consider a surface with strong electronic correlations that is modeled by a Hubbard hamiltonian (12) with hopping amplitude $J[\langle i, j\rangle$ denotes nearest neighbors $]$ and onsite interaction strength $U$.

$$
\begin{aligned}
H_{\mathrm{e}}= & -J \sum_{\langle i, j\rangle, \sigma} c_{i \sigma}^{\dagger} c_{j \sigma}+U \sum_{i}\left(n_{i \uparrow}-\frac{1}{2}\right)\left(n_{i \downarrow}-\frac{1}{2}\right) \\
& -\frac{Z_{\mathrm{p}} e^{2}}{4 \pi \epsilon_{0}} \sum_{i, \sigma} \frac{c_{i \sigma}^{\dagger} c_{i \sigma}}{\left|\vec{r}_{\mathrm{p}}(t)-\vec{R}_{i}\right|}+\sum_{\langle i, j\rangle, \sigma} W_{i j}(t) c_{i \sigma}^{\dagger} c_{j \sigma} \cdot
\end{aligned}
$$

The strength of correlations is measured by the ratio $U / J$ and is typically in the range from 0 to 10 . The second line of Eq. (12) contains the coupling of the lattice electrons located at coordinate $\vec{R}_{i}$ with a positively charged projectile of charge $Z_{p}$ that is treated classically (Ehrenfest dynamics) by solving Newton's equation for the trajectory $\mathbf{r}_{p}(t)$ under the influence of all Coulomb forces with the lattice electrons. The final term allows to improve the model by accounting for modification of the hopping rates due to the projectile according to $W_{i j}(t)=\gamma\left[W_{i i}(t)+W_{j j}(t)\right] / 2$, where $W_{i i}$ is the magnitude of the Coulomb potential of the projectile at lattice site "i" and $\gamma$ is a phenomenological parameter of the order unity [15].

The KBE (11) with the hamiltonian (12) have been solved numerically for a two-dimensional hexagonal lattice as is known e.g. for graphene [cf. top part of Fig. 12] of finite size (the number of lattice sites $L$ varies from 24 to 384 ) for a broad range of projectile velocities [15], see Fig. 11. For the correlation selfenergy $\Sigma$ several approximations were used: no selfenergy (this corresponds to the mean field or Hartree approximation), second Born (second order in the electron-electron interaction), the third order and the T-matrix
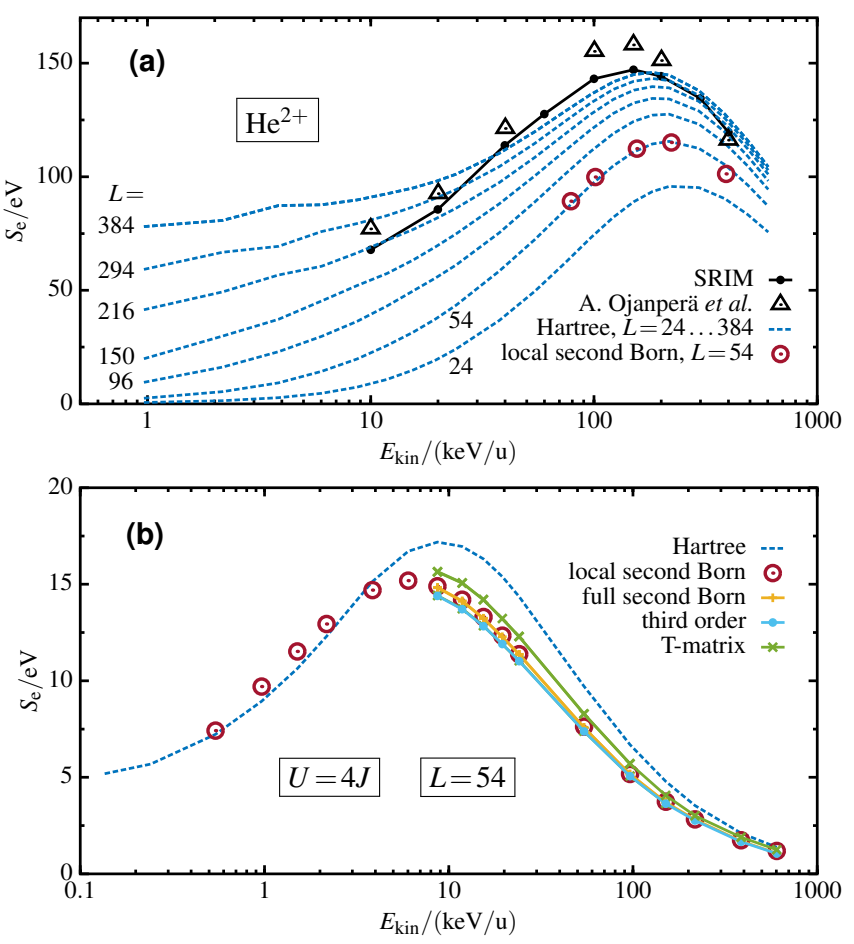

Figure 11: Energy loss of doubly charged helium ions in a graphene sheet [15]. (a) Simulation results for $U / J=1.6, J=3.15 \mathrm{eV}$ and $\gamma=0.55$ and for different cluster size $L$ are compared with SRIM and TDDFT simulations of Ojanperä et al. [151] for an infinite system. The NEGF data (lines: Hartree approximation, red symbols: second Born selfenergy) show good agreement and, in addition, extend to lower projectile energies. (b) A honeycomb cluster with $L=54$ sites, $U / J=4 J=2.8 \mathrm{eV}, \gamma=0$, is studied with different selfenergy approximations: second Born, third-order and T-matrix approximation showing a clear impact of correlations.

(strong coupling) approximation. The results demonstrate good agreement with available data from SRIM [208] and TDDFT [151] simulations for high and intermedeate impact energies, down to $10 \mathrm{keV}$ which confirms the choice of the selfenergy. The strength of the NEGF approach is that it also provides data for lower energy, as are typical for lowtemperature plasmas. Moreover, the simulations are not restricted to spatially uniform systems but are also directly applicable to finite systems such as graphene nanoclusters, nanostructured surfaces. A particular interesting example are small graphene "nanoribbons" that have a size-dependent band gap, e.g. [4,5], giving rise to promising electronic and optical properties. For an overview on plasma synthesis of nanomaterials, see Refs. 2,3. In these systems, finite size effects play an important role which is in particular true for the stopping power, as was demonstrated in the top part of Fig. 11. Finally, the NEGF approach also applies to strongly correlated materials the potential of which for plasma applications has not been explored yet.

So far we have not clarified what is the energy loss mechanism for the projectile - except for the fact that the projectile produces, within the above model, purely electronic ex- 
citations (lattice vibrations can be included straightforwardly, but this will not be of interest here, assuming that only times shorter than about $100 \mathrm{fs}$ are considered). In the following we use our time-dependent and space resolved simulations to investigate the electronic correlations effects that are excited by the projectile in a graphene-type nanostructure more in detail. Of particular interest is the local excitation of double occupancies ("doublons"), $d_{i}(t)=\left\langle\hat{n}_{i \uparrow}(t) \hat{n}_{i \downarrow}(t)\right\rangle$. These are pairs of electrons (with opposite spin projection) that occupy the same lattice site "i" and can be considered bound states, despite their repulsive Coulomb interaction. If the initial state
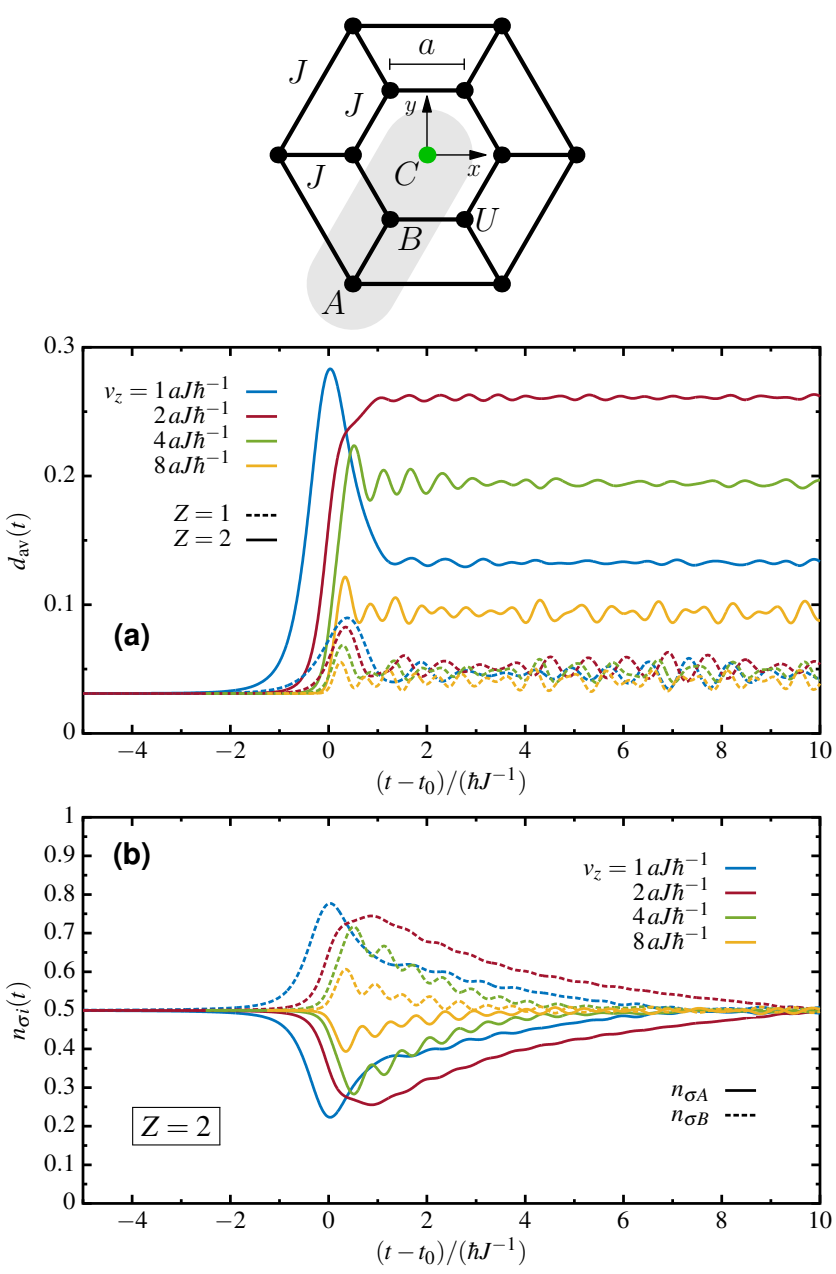

Figure 12: Time-dependent response of a strongly correlated finite honeycomb cluster ( $L=12$ sites, $U / J=10)$ to a charged projectile penetrating through the center (point $\mathrm{C}$ in top figure). (a) siteaveraged double occupation, $d_{\mathrm{av}}(t)=\frac{1}{L} \sum_{i} d_{i}(t)$, for charge $Z=1$ (dashed lines), and $Z=2$ (full lines). (b) The densities on sites A (full line) and B (dashes) closest to the projectile, for the case $Z=2$, after Ref. [186].

of the lattice is "half filled", i.e. there are as many electrons as lattice sites, this system will behave as an insulator, because any additional electron will have to sit on an already occupied site. This, however, costs an energy $U$, cf. the hamiltonian (12). An analysis of the energy spectrum reveals that this electron occupies an excited energy band ( $U$ above the lower band). If an external electric field would applied, this electron would be mobile, once it has overcome this energy gap. If the system is now hit by an energetic ion, part of this energy could be transformed into excitation of electrons to the upper Hubbard band, i.e. to the formation of doublons and of a conducting state.

This hypothesis is tested on an example in Fig. 12 where a projectile penetrates a honeycomb cluster in the center. As a result, in the cluster the electrons are forced towards the center, which is seen by a density increase on the nearest site (B) and a corresponding decrease at the next nearest site (A). Quickly after the projectile has passed through the densities return to their initial values. At the same time, the double occupation (a correlation effect) of the site $\mathrm{A}, d_{A}$, is increased as well, which is due to a transition of the whole electronic system to a higher energy eigenstate. Most importantly, this excitation is stored in the system even after the projectile has left, it only redistributes equally among the sites A and B (cf. the average that is shown by the green curve). The largest effect is observed for energies of $120 \mathrm{eV}$, in the case of protons (480 eV, for alpha particles) [186]. This way part of the energy of the projectile from the plasma is stored in non-trivial electronic excitations of the solid which might find interesting applications in the future.

We note that these simulations are very CPU time consuming. This allows to do simulations only for selected impact parameters (no averaging over trajectories), as in TDDFT simulations [151]. Also, long time scales (that describe, e.g. the coupling of electronic and phononic degrees of freedom) are not easily accessible. Also, a full quantum treatment of projectiles with internal degrees of freedom (atoms, larger ions, molecules) will require further developments. In this respect, these NEGF simulations are complementary to the quantum kinetic models of Sec. V or TDDFT simulations, cf. Sec. VIB.

\section{Ab initio NEGF (AI-NEGF)}

The NEGF simulations of ion stopping were, so far, restricted to lattice model systems, in order to systematically explore the role of electronic correlations and to investigate finite size effects in nanostructures. In order to extend this approach to a broad range of realistic materials one can couple NEGF simulations to a Kohn-Sham basis that is precomputed by a ground state DFT simulation, as was discussed in Sec. VI. In fact, such an approach was developed a few years ago by Marini and co-workers within their Yambo code [39] and provides a suitable basis for future improvements of the NEGF simulations towards realistic material properties. The public equilibrium part of the program is well suited to compute the dielectric function, optical properties of materials and was successfully tested in Kiel for graphene [209]. In the following we plan to extend the nonequilibrium part by including stopping simulations as explained in Sec. VII B. 


\section{SYNERGIES OF THE SURFACE SIMULATION APPROACHES}

Let us now discuss what kind of synergies exist between the various surface simulation methods that were listed in the central box of Fig. 7 and were discussed in Secs. IV, V, VI and VII. These methods are very different in terms of accessible length and time scales and the nature of physical approximations, therefore, an efficient and reliable combination into a single single computer code (multi-scale approach) does not seem realistic, at the moment. Nevertheless, it is very interesting to explore what synergies these concepts may possess and how they may be combined for a better understanding of selected relevant surface simulation processes. Of particular interest are, of course, such processes that can be treated with more than one method which offers the potential of cross checks, benchmarks and improvements of approximations. In table I we list such processes and discuss the potential of the surface simulation approaches.

The table shows that there exist a large range of applications of these methods to quite diverse topics. For method development and improvement the best candidates are topics where several methods have been applied in the past and where, therefore, cross checks are possible.

\section{TOWARDS INTEGRATED PLASMA-SURFACE MODELING}

After reviewing the various simulation methods for surface processes we now discuss possible ways how to integrate them into a simulation of the plasma-solid interface. In the first two parts of this section we consider a quantum kinetic theory approach to charge transfer processes across the interface. First, in Sec. IX A we consider the description of the quasistationary electric double layer that was introduced in Sec. III A and Fig. 6 and derive matching conditions between plasma and surface. After this, in Sec. IX B we outline a time-dependent approach to electron transfer between plasma ions and a solid, including electronic correlation effects. Finally, at the end of this section we briefly return to the coupling of plasma and surface simulations, in Sec. IX C, and to the issue of how to keep track of surface morphology changes, cf. Sec. IX D.

\section{A. Integrated modeling of the electric double layer}

In the previous sections we discussed the interaction of a plasma with a solid surface in terms of elementary surface collision processes. Similar to collisions in the bulk of the discharge they are characterized by collision probabilities (viz: sticking, reflection, and secondary electron emission coefficients) which have to be determined either by independent measurements or by separate quantum-mechanical calculations. In contrast to bulk processes, however, where the surrounding plasma does not affect the collision partners, in a surface collision the plasma affects the target. The parameters used to characterize a surface collision depend thus on the plasma. Taking the plasma-induced modifications of the surface into account requires a selfconsistent modeling of the mutual influence between the plasma and the solid. Such an integral modeling approach is of course very demanding involving, in general, processes and species acting on different scales. In this section we will thus exemplify the approach only for an idealistic situation, where the only physical consequence of the plasma-surface interaction is an electric response leading to the build-up of an electric double layer at the plasma-solid interface, as discussed in Sec. III A, cf. Fig. 6. First steps towards a selfconsistent description of this double layer, for a floating dielectric surface, have been made by Bronold and Fehske [214]. In this subsection we summarize and comment on their work.

The formation of an electron-depleted region on the plasma side of the interface and an electron-rich region inside or on top of the solid, depending on its electronic structure, is the most fundamental manifestation of the interaction of a solid surface with an ionized gas. It arises because electrons in the plasma outrun heavy species leading to a more efficient electron deposition due to electron absorption than electron extraction by neutralization/de-excitation of ions/radicals. That the electric response of the plasma-solid interface leads to an electric double layer, having a negative part inside the solid and a positive part inside the plasma, is known since the beginnings of gaseous electronics [215]. Ever since, however, the focus of interest has been on the plasma-based electrondepleted part of the double layer-the plasma sheath-how it merges with the quasi-neutral bulk plasma [216-220] and how it is affected by the emissive properties of the surface [221226]. The negative part of the double layer found essentially no attention. Yet it is an integral part of the electric response of the solid to the plasma.

Usually, the theoretical descriptions of the electric response of the plasma-solid interface assume that the processes inside the solid occur on time scales too fast and length scales too small to affect the plasma [227]. Based on such a view, it is thus sufficient to replace the plasma-facing solid by an object with a geometrical boundary and probabilities for electron sticking/reflection, ion neutralization, and secondary electron emission [59]. Within such an approach [228-230] it is of course impossible to investigate the plasma-induced modifications of the electronic structure of the solid, which in turn however may strongly affect the probabilities for charge transfer. We consider this to be a particularly severe drawback for the modeling of microdischarges on semiconducting substrates [231,232]. Due to the continuing miniaturization of these structures [233] the electron transit times through the plasma sheath may become comparable to the electron relaxation time inside the solid. Between subsequent electron encounters the electrons inside the solid can thus no longer equilibrate. It is hence no longer viable to describe charge transfer across the plasma-solid interface by a number of fixed surface parameters in such situations. Instead charge-transfer has to be considered as the linking part of a selfconsistent modeling of the charge dynamics on both sides of the interface. As illustrated in Fig. 13, the selfconsistent integral modeling of the electric response of the plasma-solid interface tracks elec- 


\begin{tabular}{|c|c|c|c|}
\hline Process & Methods & Pros & Cons \\
\hline Ion stopping & $\begin{array}{l}\text { MD } \\
\text { DFT } \\
\text { TDDFT } \\
\text { NEGF } \\
\text { AI-NEGF }\end{array}$ & $\begin{array}{l}\text { large system } \\
\text { accounts for electronic structure } \\
\text { electronically non-adiabatic } \\
\text { electronic correlations } \\
\text { Ref. 15, Sec. VII B } \\
\text { accounts for electronic structure, } \\
\text { possible with Yambo, Sec. VII C [39] }\end{array}$ & $\begin{array}{l}\text { el. adiabatic, unknown accuracy of empirical forces } \\
\text { el. adiabatic, small system, limited accuracy of approx.XC } \\
\text { kinetic energy limits due to pseudopotentials } \\
\text { unknown accuracy of approx. XC } \\
\text { only electronic stopping from selected trajectories } \\
\text { finite system, Hubbard-type model } \\
\text { classical ion (Ehrenfest dynamics) } \\
\text { small systems, approximate electronic correlations } \\
\text { not yet tested for plasma-surface applications }\end{array}$ \\
\hline Ion neutralization & $\begin{array}{l}\text { QBE } \\
\text { TDDFT }\end{array}$ & $\begin{array}{l}\text { correlations in projectile, Ref. } 210 \\
\text { accounts for electronic structure }\end{array}$ & $\begin{array}{l}\text { model surface } \\
\text { unknown/limited accuracy of adiabatic approx to XC }\end{array}$ \\
\hline $\begin{array}{l}\text { Electron sticking/ } \\
\text { absorption }\end{array}$ & QBE & Ref. 20 & model surface \\
\hline SEE & QBE & correlations in projectile, Sec. V & model surface \\
\hline Atom sticking & $\begin{array}{l}\text { MD } \\
\text { QBE }\end{array}$ & $\begin{array}{l}\text { Refs. } 54,58, \text { Sec. IV B } \\
\text { Ref. } 211\end{array}$ & $\begin{array}{l}\text { classical, no electronic effects } \\
\text { model systems }\end{array}$ \\
\hline Cluster/layer growth & $\begin{array}{l}\text { MD } \\
\text { KMC }\end{array}$ & $\begin{array}{l}\text { Refs. 44,52, Sec. IV A } \\
\text { Refs. 32,212 }\end{array}$ & $\begin{array}{l}\text { classical, no electronic effects } \\
\text { phenomenological }\end{array}$ \\
\hline Surface reactions & $\begin{array}{l}\text { DFT } \\
\text { TDDFT }\end{array}$ & $\begin{array}{l}\text { accounts for electronic structure } \\
\text { electron. non-adiabatic [213] }\end{array}$ & $\begin{array}{l}\text { el. adiabatic, limited accuracy of approx.XC } \\
\text { small system, few selected trajectories, } \\
\text { unknown/limited accuracy of adiabatic approx. to XC }\end{array}$ \\
\hline Sputtering & MD & large system, Ref. 13 & el. adiabatic, unknown accuracy of empirical forces \\
\hline
\end{tabular}

Table I: Selection of important plasma-surface processes, main surface science methods (as listed in Fig. 7) as well as their quality and limitations. For applications discussed in this paper, the corresponding section number is given. "XC" denotes exchange correlation functional, "el. adiabatic": electronically adiabatic.

trons $\left(e^{-}\right)$and ions $\left(i^{+}\right)$generated by impact ionization inside the plasma to the inside of the solid, where they recombineafter energy- and momentum-relaxation-either radiatively by band-to-band transitions or non-radiatively via trap states as conduction band electrons $\left(e_{*}^{-}\right)$and valence band holes $\left(h^{+}\right)$. The plasma source is thus linked to the plasma sink inside the solid. We expect an understanding of this link to be central for the progress of future efforts combining gaseous and semiconductor electronics [234,235].

To get an idea about how to organize the self-consistent integral modeling of the electric response of the plasma-solid interface, let us consider a dielectric solid facing a plasma [214].
The modeling then is based on two sets of spatially separated Boltzmann equations, one for the electrons and ions inside the plasma and one for the conduction band electrons and valence band holes inside the solid (using an electron-hole picture inside the solid simplifies the calculations). Defining a species index $s=e, i, *, h$ to denote electrons, ions, conduction band electrons, and valence band holes, the Boltzmann equations for the quasi-stationary distribution functions $F_{s}^{\gtrless}(z, E, \vec{K})$ for left and right moving particles can be generally written as (measuring length in Bohr radii and energy in Rydbergs) [214]

$$
\left[ \pm v_{s}(z, E, \vec{K}) \frac{\partial}{\partial z}+\gamma_{s}^{\gtrless}\left[F_{s^{\prime}}^{>}, F_{s^{\prime}}^{<}\right]\right] F_{s}^{\gtrless}(z, E, \vec{K})=\Phi_{s}^{\gtrless}\left[F_{s}^{>}, F_{s}^{<}, F_{s^{\prime}}^{>}, F_{s^{\prime}}^{<}\right]
$$

with

$$
v_{s}(z, E, \vec{K})=2\left(\frac{m_{e}}{m_{s}}\left[E-U_{s}(z)\right]-\left(\frac{m_{e}}{m_{s}} \vec{K}\right)^{2}\right)^{1 / 2}
$$

the velocity of the particles normal to the (planar) interface at $z=0$. Here, $z, E$, and $\vec{K}$ denote the distance from the interface, the total energy, and the lateral momentum of the particles. The functions $U_{s}(z)$ are shown in Fig. 14 (together with other quantities relevant for the description of the electric double layer). They define the regions in $z-E$ space where the respective species move freely [214]. The alignment of the electronic energies of the solid with the ones of the plasma is controlled by the electron affinity $\chi$ and the energy gap $E_{g}$. The functions $\gamma_{s}^{\gtrless}$ and $\Phi_{s}^{\gtrless}$ denoting, respectively, the rates for out-scattering and the in-scattering collision integrals depend on the scattering process.

The Boltzmann equations themselves are not sufficient. They have to be augmented by matching and boundary conditions for the distribution functions, the former applying to $z=0$ and the latter to the asymptotic regions of the inter- 


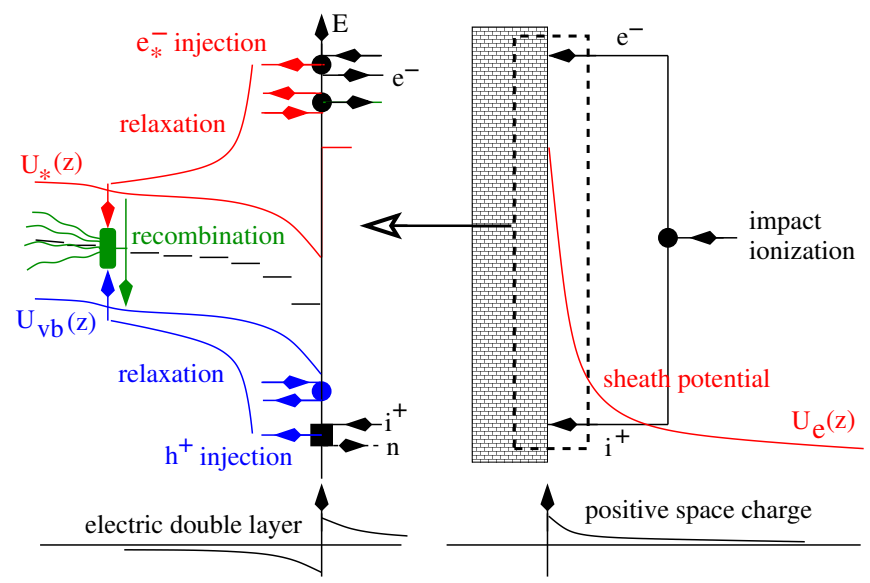

Figure 13: (Color online) Illustration of the electric response of a floating dielectric plasma-solid interface. On the right is shown the traditional modeling treating the solid as a black box, characterized by surface parameters such as the electron sticking coefficient and the ion wall recombination probability. The left depicts the processes actually taking place inside the solid when plasma is destroyed. Electrons $\left(e^{-}\right)$and ions $\left(i^{+}\right)$, generated by impact ionization in the plasma, hit the dielectric solid, thereby injecting conduction band electrons $\left(e_{*}^{-}\right)$and valence band holes $\left(h^{+}\right)$, which after relaxation may either recombine non-radiatively or radiatively. As a result, electron depletion occurs in front of the surface leading to a positive space charge which in turn is balanced by a negative space charge inside the solid (electric double layer). The selfconsistent kinetic modeling [214] treats impact ionization in the plasma and recombination in the solid on an equal footing by tracing the ambipolar charge transport across the interface, allowing for quantummechanical reflection/transmission and charge transport/relaxation on both sides of the interface.

face. For a dielectric plasma-solid interface without interface/surface states the matching conditions for the electron distribution functions become $(E>0)$

$$
\begin{aligned}
F_{e, *}^{>,<}(0, E, \vec{K}) & =R(E, \vec{K}) F_{e, *}^{<,>}(0, E, \vec{K}) \\
& +[1-R(E, \vec{K})] F_{*, e}^{>,<}(0, E, \vec{K}),
\end{aligned}
$$

while the hole and ion distribution functions are connected by $\left(E>E_{g}+\chi\right)$

$$
\begin{aligned}
& F_{h}^{<}(0, E, \vec{K})=F_{h}^{>}(0, E, \vec{K})+\alpha S_{h}^{<}(E, \vec{K}), \\
& F_{i}^{>}(0, E, \vec{K})=(1-\alpha) F_{i}^{<}(0, E, \vec{K}) .
\end{aligned}
$$

The function $R(E, \vec{K})$ is the quantum-mechanical reflection coefficient for electrons due to the surface potential which can be, for instance, modelled by a three-dimensional potential step with a mismatch in the electron mass arising from the difference between the effective electron mass $m_{e}^{*}$ inside the solid and the electron mass $m_{e}$ in the plasma, $\alpha$ is the neutralization probability for an ion at the interface, and $S_{h}^{<}(E, \vec{K})$ is a function specifying the accompanying hole injection into the valence band of the dielectric. An illustration of the matching conditions, coupling the distribution functions of the conduction band electrons $\left(e_{*}^{-}\right)$and valence band holes $\left(h^{+}\right)$in the solid with the distribution functions of the electrons $\left(e^{-}\right)$and ions $\left(i^{+}\right)$in the plasma, is included in Fig. 13. The particular form of $S_{h}^{<}(E, \vec{K})$, into which $F_{i}^{<}$enters [214], depends on the neutralization process. In case the neutralization induces also secondary electron emission, the matching condition for the electron distribution functions has to be augmented by a function $S_{e}^{>}(E, \vec{K})$. With the Poisson equation for the electric potential energy $U_{c}(z)$ (again given in atomic units),

$$
\frac{d}{d z} \varepsilon(z) \frac{d}{d z} U_{c}(z)=8 \pi\left[\rho_{w}(z) \theta(-z)-\rho_{p}(z) \theta(z)\right]
$$

where $\rho_{w}(z)=n_{*}(z)-n_{h}(z)-n_{D}+n_{A}>0$ and $\rho_{p}(z)=$ $n_{i}(z)-n_{e}(z)>0$ are, respectively, the charge densities inside the surface (assumed to contain donors and acceptors with concentrations $n_{D}$ and $n_{A}$ ) and the plasma, to be obtained from the distribution functions by integration,

$$
n_{s}(z)=\int \frac{d E d^{2} K}{(2 \pi)^{3}} \frac{F_{s}^{>}(z, E, \vec{K})+F_{s}^{<}(z, E, \vec{K})}{v_{s}(z, E, \vec{K})},
$$

and the matching conditions for $U_{c}(z)$ at $z=0$, Eqs. 13-16 form a closed set of equations for the distribution functions and the electric potential energy provided they are augmented by boundary conditions far away from the interface, at $z=$ $z_{b}<0$ and $z=z_{w}>0$ (see Fig. 14).

Essential for the selfconsistent description of the electric response is the implementation of the boundary conditions far away from the interface. It depends on how the interface, and hence the electric double layer, is electrically connected to the outside. For an electrically floating interface the double layer is embedded between two quasi-neutral, field-free regions.

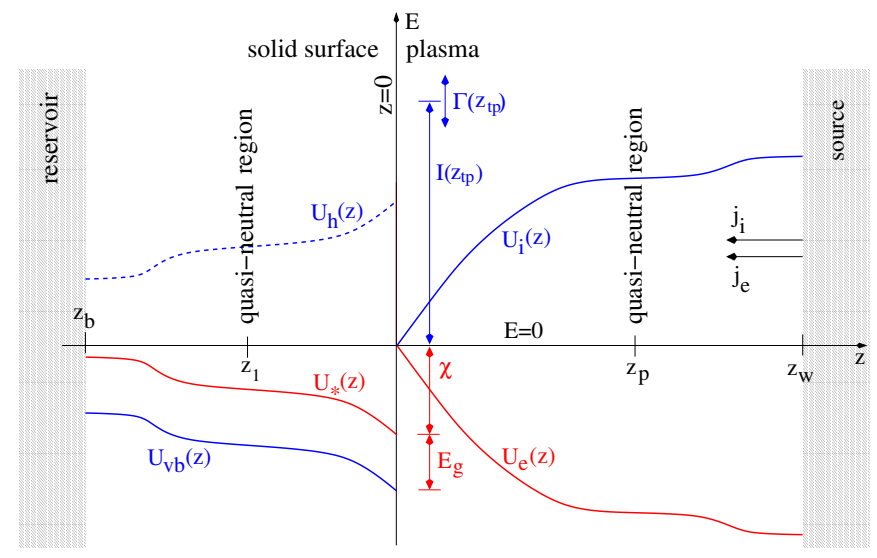

Figure 14: Notation used for the description of an electric double layer at a floating dielectric plasma-solid interface with negative space charge inside the solid and positive space charge in front of it. Shown are the edges of the conduction $\left(U_{*}\right)$ and valence $\left(U_{v b}\right)$ bands, the edge for the motion of valence band holes $\left(U_{h}\right)$, the potential energies for electrons $\left(U_{e}\right)$ and ions $\left(U_{i}\right)$ on the plasma side, and the energetic range, specified by the ion's ionization energy $I$ and its broadening $\Gamma$, in which hole injection occurs due to the neutralization of ions at the interface. Source, reservoir, and quasi-neutral regions are indicated as they will arise in the course of the calculation (adopted from Ref. 214). 


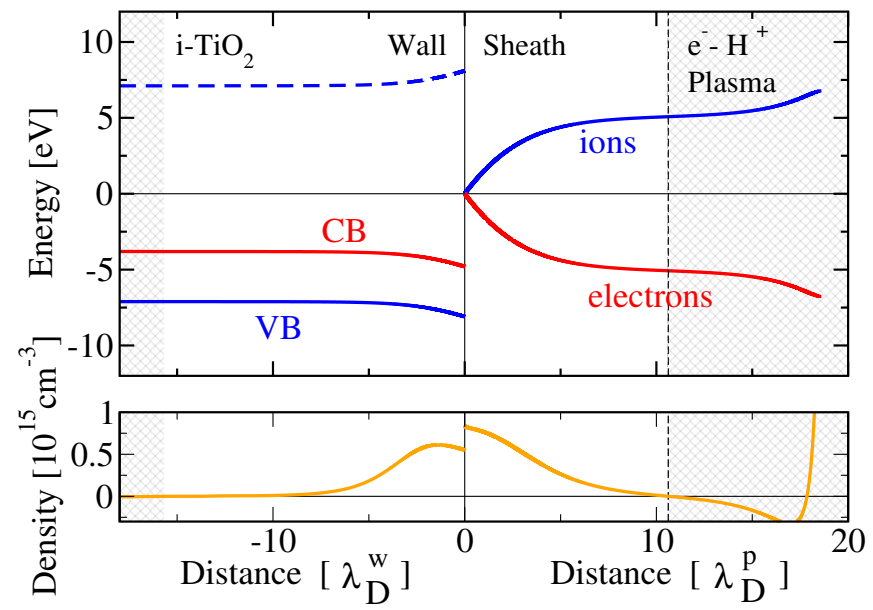

Figure 15: Upper panel: Edges for the conduction and valence bands (solid red and blue lines), the free hole motion (dashed blue lines), and the potential energies for electrons and ions on the plasma side (also indicated by solid red and blue lines) for an intrinsic $\mathrm{TiO}_{2}$ surface in contact with an hydrogen plasma. Shaded regions indicate respectively the reservoir and source which have been set up to provide the correct physical boundary conditions for the double layer. Lower panel: Density profiles $\rho_{w}(z)=\left[n_{*}(z)-n_{h}(z)\right] \theta(-z)$ and $\rho_{p}(z)=\left[n_{i}(z)-n_{e}(z)\right] \theta(z)$. By definition they are both positive outside the shaded regions, that is, in the regions which are physically relevant. The interface is collisionless on both sides and perfectly absorbing. Material and plasma parameters are $\chi=4.8 \mathrm{eV}$, $E_{g}=3.3 \mathrm{eV}, m_{e}^{*}=m_{e}, m_{h}=0.8 m_{e}, \varepsilon=6, I=13.6 \mathrm{eV}$, $\Gamma=2 \mathrm{eV}, k_{B} T_{*}=k_{B} T_{h}=0.2 \mathrm{eV}, k_{B} T_{e}=10 k_{B} T_{i}=2 \mathrm{eV}$. Due to the somewhat unrealistic temperatures of the charges on both sides of the interface (required to stabilize the numerics) the Debye screening length on the solid side $\lambda_{D}^{w}=2.2 \cdot 10^{-6} \mathrm{~cm}$ is comparable to the Debye screening length on the plasma side $\lambda_{D}^{p}=1.6 \cdot 10^{-6} \mathrm{~cm}$. Due to the absence of collisions the numerical values, however, should not be taken literally. A collisional theory would produce different density and potential profiles.

The distribution functions in these regions are of course unknown. They are themselves the result of the electric response and can thus not be used as boundary conditions for the Boltzmann equations. A possibility to overcome this complication is to enforce two inflection points in the profile of $U_{C}(z) \mathrm{mim}-$ icking the quasi-neutral, field-free surroundings of the double layer, see Fig. 14. The price to be paid comes in the form of eight parameters: Four boundary densities $\left(n_{b *}, n_{b h}, n_{s e}\right.$, and $n_{s i}$ ), for each of the charged species considered, and four spatial coordinates $\left(z_{b}, z_{1}, z_{p}\right.$, and $\left.z_{w}\right)$, two of them denote the positions of the inflection points $\left(z_{1}, z_{p}\right)$, one the position of the particle reservoir in the solid $\left(z_{b}\right)$, and one the position of the source $\left(z_{w}\right)$ in the plasma. The adjustment of the distribution functions to the interface can then be emulated without simulating the bulk of the solid and the plasma.

The framework outlined indicates what is needed for an integral modeling of the electric response. Besides specifying collision processes on both sides of the interface, matching and boundary conditions for the distribution functions have to be worked out-based on, respectively, the emissive properties of the plasma-solid interface and its asymptotics on both sides.
To gain first insights into the selfconsistent electric response of a plasma-solid interface Bronold and Fehske [214] applied the equations listed above to a collisionless, perfectly absorbing interface. By ignoring the collision integrals, the lateral momentum $\vec{K}$ could be eliminated from all equations, including the matching conditions. In addition, the solutions of the Boltzmann equations turned out to be no longer explicit functions of $z$ but only functions of $E$ and $U_{C}(z)$. The first integral of the Poisson equation could thus be obtained analytically, greatly simplifying the further numerical treatment. The selfconsistent embedding finally led to four nonlinear algebraic equations for the potential energies $U_{C}\left(z_{b}\right), U_{C}\left(z_{1}\right), U_{C}\left(z_{p}\right)$, and $U_{C}\left(z_{w}\right)$ which could be solved numerically with moderate effort. Results for the perfectly absorbing, collisionless interface-for parameters applicable to intrinsic $\mathrm{TiO}_{2}$ in contact with an hydrogen plasma-are shown in Fig. 15.

In a collisionless theory recombination and generation of electron-hole pairs inside the solid are excluded. Nothing balances thus-strictly speaking-the permanent influx of electrons and ions onto the interface. For a quasi-stationary regime to develop it is necessary to introduce by hand a recombination condition. The condition employed in Ref. [214] utilized the fact that intraband energy and momentum relaxation is much faster than interband recombination. The electron and hole densities inside the solid can thus be split into thermalized/trapped $\left[n_{*, h}^{t}(z)\right]$ and free parts $\left[n_{*, h}^{j}(z)\right]$, with only the former acting as a source in the Poisson equation, and the latter assumed to cancel once integrated over a spatial strip extending from the inflection point $z_{1}<0$ inside the solid to the interface at $z=0$,

$$
\int_{z_{1}}^{0} d z \rho_{w}^{j}(z)=\int_{z_{1}}^{0} d z\left[n_{*}^{j}(z)-n_{h}^{j}(z)\right]=0 .
$$

With this postulate the collisionless theory provided in total eight equations for the eight parameters mentioned above. A selfconsistent description of the electric response, indicated by a selfconsistent embedding of the electric double layer between two quasi-neutral, field-free regions, could thus be realized without taking collisions into account.

The absence of collisions can be at most justified on the plasma side, where electrons are strongly depleted and hence nearly collisionless, and ions are only subject to ion-neutral collisions which however have to be considered only in particular situations [236-238] or when a very precise description of the plasma sheath is required [239]. Hence, on the plasma side ignoring or including collisions is essentially a question of how accurate one wants to describe the plasma sheath. On the solid side of the interface, however, neglecting collisions makes the theory conceptually incomplete. Without recombination, either radiatively or non-radiatively, both involving collisions between conduction band electrons and valence band holes, with the latter mediated by trap states (see Fig. 13), no quasi-stationarity can be achieved because nothing balances the injection of conduction band electrons and valence band holes from the plasma. The carrier concentration inside the solid would just grow indefinitely. Future work has thus to include collisions, at least on the solid side of the interface. The ad-hoc condition (19) could then be replaced 
by a consistent truncation of the perturbation theory treatment of charge relaxation and recombination. Work in this direction is in progress.

In this subsection we discussed a general framework extending the kinetic modeling of the charge dynamics of the plasma to the inside of the solid. Within such an approach the electric modification of the plasma-solid interface-the buildup of the electric double layer-can be described selfconsistently, treating the plasma-based positive and the solid-based negative part of the double layer on an equal footing. In particular for hybrid electronics, using arrays of microdischarges integrated on semiconducting substrates, it may be necessary to adopt this type of integral modeling. In discharges used for materials processing, chemical and structural responses of the interface, involving the formation of adsorption layers and/or the sputtering of the outer layers of the interface, are of course intimately coupled to the electric response. To include them into the framework we just described is in principle possible. Staying at the level of Boltzmann equations is rather advantageous at this point. For each species of interest one has to add a Boltzmann equation with appropriate collision terms and matching/boundary conditions. Ways to treat rough and disordered interfaces are also conceivable. The numerical effort however will be rather high. An integral modeling, taking the electric, chemical, and structural response of the plasmasolid interface selfconsistently into account will be possible using high performance computing.

The limitations of the present approach are mostly due to the simplified treatment of the solid. The related processes in the surface material require $a b$ initio modeling. In the next section such an approach that is based on nonequilibrium Green functions will be outlined. At the same time, there the treatment of the plasma particles will be substantially simplified compared to the present treatment. So both methods have complementary features.

\section{B. Integrated modeling of ion-surface interaction}

In the previous subsection the traditional plasma physics approach that assumes that the processes inside the solid occur on too short (small) time (length) scales to affect the plasma [227]. However, if the plasma impact is spatially localized and due to rare events (such as the impact of a projectiles) substantial deviations from such an approach have to be expected. It is, therefore, of interest to consider a space and time resolved description which, in principle allows to verify the above hypothesis or, on the opposite, to capture processes that are missed by it. While the former approach can be understood as based on space and time averaging over the scales of the solid - which is essentially a mean field description, the latter concept takes into account correlation effects and fluctuations around mean values, e.g. [240].

Here, we develop a quantum kinetic description of the coupled electron-ion dynamics across the plasma interface generalizing the methods described in Sec. VII. We start from the second-quantized many-body Hamiltonian for the electrons in the interface and separate the system into a plasma $(p)$ and solid surface part $(s)$ [we denote $\Omega=\{p, s\}$ and do not write the spin index explicitly],

$$
\begin{aligned}
H_{\text {interface }}= & \sum_{\alpha \beta \in \Omega} \sum_{i j} H_{i j}^{\alpha \beta}(t) c_{i}^{\alpha \dagger} c_{j}^{\beta}+ \\
& \frac{1}{2} \sum_{\alpha \beta \gamma \delta \in \Omega} \sum_{i j k l} W_{i j k l}^{\alpha \beta \gamma \delta} c_{i}^{\alpha \dagger} c_{j}^{\beta \dagger} c_{k}^{\gamma} c_{l}^{\delta} .
\end{aligned}
$$

Here, the operator $c_{i}^{\alpha \dagger}\left(c_{i}^{\alpha}\right)$ creates (annihilates) an electron in the state $i$ of part $\alpha$. The one-particle Hamiltonian $H(t)$ contains the electron's kinetic and the time-dependent potential energy, and $W$ accounts for all possible electron-electron Coulomb interactions within and between the two parts.

Considering individual energetic plasma ions, which penetrate into the solid and undergo scattering and stopping in the surface layers, we describe the system (20) by a one-particle nonequilibrium Green function (NEGF) $G_{i j}^{\alpha \beta}\left(t, t^{\prime}\right)$, as introduced in Sec. VII A, but here with an additional $2 \times 2$ matrix structure $(\alpha, \beta=\{p, s\})$,

$$
\begin{aligned}
G_{i j}^{\alpha \beta}\left(t, t^{\prime}\right) & =-i\left\langle T_{C} c_{i}^{\alpha}(t) c_{j}^{\beta \dagger}\left(t^{\prime}\right)\right\rangle, \\
\rho_{i j}^{\alpha \beta}(t) & =-i G_{j i}^{\beta \alpha}\left(t, t^{+}\right),
\end{aligned}
$$

e.g., Refs. [37,202], and the time-diagonal elements provide the density matrix (22), as discussed in Sec. VII A. The diagonal elements, $\rho_{i j}^{p p}\left[\rho_{i j}^{s S}\right]$, refer to the plasma part, describing the dynamics of free electrons and electrons bound in the ion [to the solid part, describing electrons in bound states of the solid surface]. Moreover, the density matrix component $\rho_{i j}^{p s}$ is related to charge transfer processes between plasma and solid and will be of special interest in the following.

The equations of motion for the NEGF are the generalization of Eq. (11) to the interface

$$
\begin{aligned}
i \partial_{t} G_{i j}^{\alpha \beta}\left(t, t^{\prime}\right)- & \sum_{\delta \in \Omega, k} H_{i k}^{\alpha \delta}(t) G_{k j}^{\delta \beta}\left(t, t^{\prime}\right)=\delta_{i j}^{\alpha \beta} \delta_{C}\left(t, t^{\prime}\right)+ \\
& \sum_{\delta \in \Omega, k} \int_{C} d \bar{t} \Sigma_{i k}^{\alpha \delta}[W, G](t, \bar{t}) G_{k j}^{\delta \beta}\left(\bar{t}, t^{\prime}\right),(23)
\end{aligned}
$$

where the self-energy $\Sigma^{\alpha \beta}\left(t, t^{\prime}\right)$ describes the interaction between the electrons and with phonons. Even though a complete solution of the KBE (23) for real materials and with a full quantum treatment of the plasma electrons is out of reach, these equations provide the rigorous starting point for the development of consistent approximations. In the following we show how it is possible to include the electronic states of the ion via an embedding self-energy approach [202], where resonant (neutralization and ionization) processes can be studied. While this embedding approach is based on a formal decoupling of the surface and plasma parts of the KBE, it retains one-electron charge transfer in the Hamiltonian $H^{s p}$, cf. Eq. (26), see below. A closed description of the solid can be maintained if correlations in the plasma part and the feedback of the solid on the plasma can be neglected, i.e., for $\Sigma^{s p}=\Sigma^{p p}=0$. This is usually well fulfilled, except for atmospheric pressure plasmas where small correlation corrections should be taken into account. Then, the KBE (11) for 
the plasma part simplify to

$$
\sum_{k}\left\{i \partial_{t} \delta_{i k}-H_{i k}^{p p}(t)\right\} g_{k j}^{p p}\left(t, t^{\prime}\right)=\delta_{i j} \delta_{C}\left(t, t^{\prime}\right),
$$

where, with the solution $g^{p p}\left(t, t^{\prime}\right)$ we denote the NEGF of the electrons inside the plasma ion, and the time dependence of $H^{p p}(t)$ accounts for possible parametric changes of the energy levels (e.g., as function of the distance of the ion from the surface).

The main result is a closed equation for $G^{s s}\left(t, t^{\prime}\right)$ :

$$
\begin{aligned}
& \sum_{k}\left\{i \partial_{t} \delta_{i k}-H_{i k}^{s s}(t)\right\} G_{k j}^{s s}\left(t, t^{\prime}\right)=\delta_{i j} \delta_{C}\left(t, t^{\prime}\right)+ \\
& \sum_{k} \int_{C} d \bar{t}\left\{\Sigma_{i k}^{c t}(t, \bar{t})+\Sigma_{i k}^{s s}\left[G^{s s}\right](t, \bar{t})\right\} G_{k j}^{s s}\left(\bar{t}, t^{\prime}\right),
\end{aligned}
$$

with the charge transfer (or embedding) self-energy that involves the charge transfer hamiltonian

$$
\begin{aligned}
\Sigma_{i j}^{c t}\left(t, t^{\prime}\right) & =\sum_{k l} H_{i k}^{s p}(t) g_{k l}^{p p}\left(t, t^{\prime}\right) H_{l j}^{p s}\left(t^{\prime}\right), \\
H_{i j}^{s p}(t) & =\int d^{3} r \phi_{i}^{s}(\vec{r})(T+V) \phi_{j}^{p}(\vec{r} ; t) .
\end{aligned}
$$

Equation (25) shows how the many-body description of an isolated (but correlated) solid is altered by the presence of the electronic states of a plasma ion, with the latter giving rise to an additional self-energy $\Sigma^{\mathrm{ct}}\left(t, t^{\prime}\right)$. While, for $\Sigma^{\mathrm{ct}}=0$, the KBE (25) conserves the particle number [for a conserving approximation of the self-energy $\Sigma^{s s}$, such as HartreeFock, second order Born or GW], the inclusion of the embedding self-energy explicitly allows for time-dependent changes of the particle number in the solid and thus accounts for ion charging and neutralization effects. For the practical solution of Eq. (25), the charge transfer Hamiltonian $H^{s p}(t)$ has to be computed by selecting the relevant electronic transitions between solid and plasma and computing the matrix elements of the kinetic and potential energy operators $T$ and $V$, with the electronic single-particle wave functions $\phi^{s}\left(\phi^{p}\right)$ in the solid (ion).

To test whether this approach can be applied to the plasmasolid interface we use the same concept as for the computation of the stopping power in Sec. VII B. We use, as a model, a nanostructured solid represented by a chain of 20 lattice sites at half filling (i.e. containing 20 electrons). The charge transfer selfenergy (26) is parametrized by a time-dependent Gaussian coupling term, $\gamma(t)=\gamma_{0} \mathrm{e}^{-\left(t-t_{0}\right)^{2} /\left(2 \tau^{2}\right)}$ that models the approach and elastic scattering of a projcetile. In Figure 16 we analyze how the charge transfere changes upon variation of the amplitude $\gamma_{0}$ and interaction duration $\tau$. Interestingly, the charge transfer dependes non-monotonically on the amplitude (see top right part of the figure) and, a large couplings, undergoes strong oscillations. On the other hand, increasing the duration of the interaction (bottom right figure) enhances the charge transfer.

With this we have formulated the general NEGF framework for integrated plasma-surface modeling. This approach is largely complementary to the one developed in Sec. IX A: There the electronic double layer and charge transfer processes along the interface were studied. Here we focused on resonant electron transfer processes attempting an accurate description of the solid. However, the plasma particles were treated on a simplified level. Ultimately, a combination of both approaches will be needed.
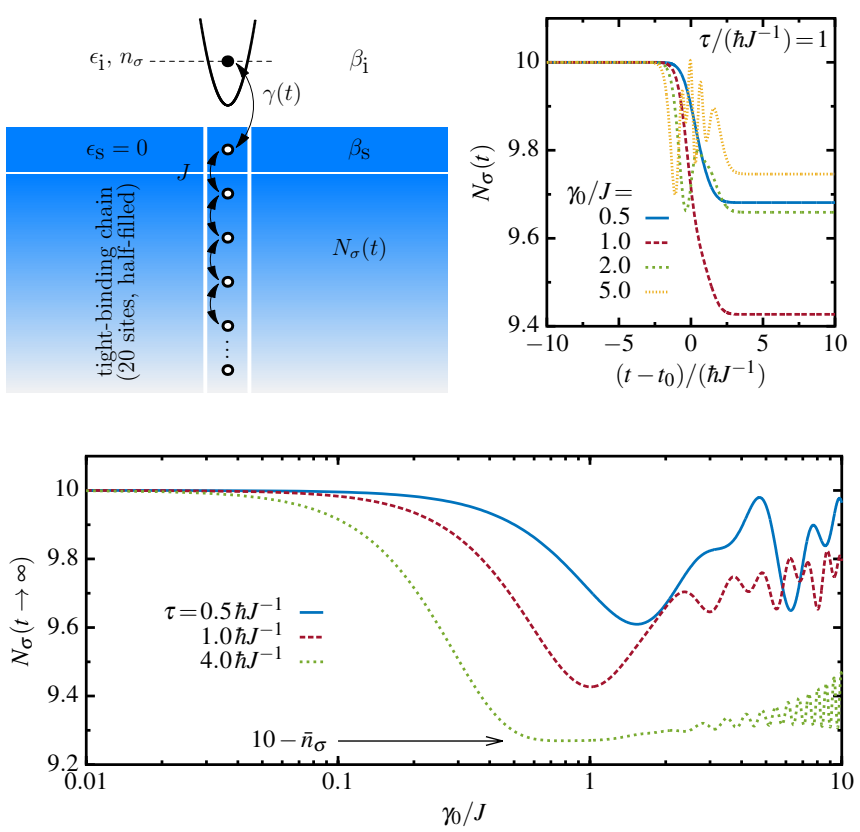

Figure 16: Example calculation illustrating the embedding scheme An initially half-filled tight-binding chain (20 sites, nearest-neighbor hopping $J$, inverse temperature $\beta_{\mathrm{s}}=100 J^{-1}$ ) is coupled via a timedependent parameter $\gamma(t)=\gamma_{0} \mathrm{e}^{-\left(t-t_{0}\right)^{2} /\left(2 \tau^{2}\right)}$ to an external energy level $\epsilon_{\mathrm{i}}=+J$ giving rise to the transfer of charge. The initial occupation of the energy level is given by $n_{\sigma}=0.269$ (corresponding to an inverse temperature of $\beta_{\mathrm{i}}=1 J^{-1}$ ) and $\bar{n}_{\sigma}=1-n_{\sigma}$. Furthermore, $N_{\sigma}(t)=\sum_{i} n_{i \sigma}(t)$ denotes the total density on the chain.

\section{Coupling plasma and surface simulations}

Returning to the overview given in Fig. 7, the coupling between surface and plasma simulations proceeds via the fluxes of particles, momentum and energy. Fluxes of neutrals, electrons and ions from the plasma, $\mathbf{J}_{a}^{p}$, have to be provided by plasma simulations and serve as an input for surface simulations. Vice versa, surface simulations, ultimately have to provide the the energy or momentum resolved fluxes, $\mathbf{J}_{a}^{s}$, of atoms, electrons and ions that leave the surface.

In the previous sections we have discussed examples of particle fluxes that are produced by surface science simulations. The first example are the fluxes of neutral gas atoms that are scattered from a metal surface and which are obtained from a combined MD-rate equations model as discussed in Sec. IV B. The result of these simulations is the sticking probability in dependence of impact energy and angle of incidence and lattice temperature, $R_{s t}\left(E_{i n}, \theta_{i n} ; T_{s}\right)$, [58]. Furthermore, 
these simulations also yield the energy distribution of the reflected atoms. These results can be directly used in PIC-MCC simulations that trace the dynamics of (fast) neutrals, in addition to electrons and ions. Based on the sticking probabilities, a Monte Carlo procedure can be used to determine if the particle is reflected back into the plasma or if it is adsorbed on the surface. In the former case, the energy distribution of the reflected atoms provides valuable information on the collision process.

The second example is secondary electron emission (SEE) which is of key relevance, as we discussed in Sec. I, cf. Fig. 4. In this paper (Sec. V) we have presented quantum kinetic results for the energy resolved SEE coefficient. The present approach is expected to be more accurate and potentially more general than previous models and thus may serve as a valuable input for PIC-MCC simulations.

\section{Taking into account plasma-induced surface morphology changes}

In Sec. IV A simulations of metal cluster growth on a polymer substrate were described. Using selective process acceleration the MD simulations could be extended to several minutes. The resulting plasma-induced adsorbate layer strongly differs from the original "clean" surface and can be used to re-compute the electronic structure of bulk and surface states via DFT as indicated in Fig. 7. Furthermore, as was discussed in Sec. II, such a modified ("dirty") surface reacts very differently on the plasma contact leading, in particular, to strongly enhanced secondary electron emission. This could be investigated in detail, e.g. by means of TDDFT (Sec. VIB) using the pre-computed surface morphology as the starting point. Alternatively, this information can be used as input for quantum kinetic simulations as described in Sec. $\mathrm{V}$ which requires to modify the employed model of the solid. Moreover, the MD simulation of neutral atom scattering, cf. Sec. IV B can be repeated using the plasma-modified surface as an input. This will directly impact the plasma properties via the modified fluxes $\mathbf{J}_{a}^{s}$, as discussed in Sec. IX C.

\section{CONCLUSION AND OUTLOOK}

In this paper we considered the interaction of lowtemperature plasmas with a solid surface, discussed the broad variety of physical and chemical processes [Fig. 6] and argued that the mutual interaction between both sides requires to develop closely coupled plasma-surface simulations. We presented an overview on a research poject that is under way at Kiel University, in collaboration with scientists from Greifswald, that aims, aomng others, at developing such simulations. Such an approach has the potential for major advances of this field because most of the currently used models are phenomenological using surface coefficients that are poorly known, both experimentally and theoretically. Moreover, these parameters-even if they exist-may carry an (unknown) dependence on the surface conditions or the plasma parameters. We discussed the simulation approaches that are required to treat the plasma and the solid side of the interface and then listed the methods that are suitable to simulate particle, energy and momentum fluxes across the interface, cf. Fig. 7. We highlighted four groups of methods: semiclassical approaches such as MD and $\mathrm{KMC}$, quantum Boltzmann equation-based models and ab initio approaches that are either based on density functional theory or on nonequilibrium Green functions.

In Sec. IV we discussed two examples of semiclassical molecular dynamics that use accurate force fields to simulate surface processes involving neutral plasma particles. The main challenge here is to extend these simulations that typcially use a time step on the order of one femtosecond to experimentally relevant times of minutes [51]. This can be achieved via selective process acceleration - which was demonstrated for the metal cluster growth on a polymer surface. Here synchronized acceleration of metal atom diffusion and deposition allows to extend the simulations by more than nine orders of magnitude up to several minutes [44]. The second approach that was discussed was dynamical freezout of collective modes (DFCM) [51,54]. Here MD simulations were used to reduce the dynamics of atom desorption on a metal surface to a small set of collective modes that obey coupled rate equations. These rate equations completely describe the sticking behavior at time scales larger than a few tens of picoseconds. There are various ways how to extend the present idea. If the surface is inhomogeneous, a straightforward generalization would be to include the space dependence into the densities and the rates. Then, the rate equations turn into hydrodynamic equations. Furthermore, the effect of a plasma environment, such as characteristic particle fluxes or an adsorbate-covered surface, are straightforwardly included into our scheme, as discussed in Ref. 54.

However, semiclassical MD has a limited sphere of applicabiity. In particular for the description of electrons and ions crossing the interface, semiclassical MD fails, and quantum approaches are necessary. This concerns the neutralization of low-energy ions, e.g. [210] and their stopping in the solid, as well as the electron dynamics across the interface, e.g. [20]. Here nonequlibrium quantum methods such as the quantum Boltzmann equation, density functional theory and nonequlibrium Green functions simulations, e.g. [14,15,37] are the methods of choice which we discussed in Secs. V, VI and VII. In the present article we attempted at giving a comprehensive overview on these methods regarding their existing applications to and future potential for accurately simulating plasma surface interaction.

At the same time, these methods have been developed, so far, almost independently of each other, but we hope that the present work will stimulate future comparisons and combinations. Each of these quantum methods, in particular timedependent DFT and NEGF, is computationally extremely expensive, and still each of them has significant limitations, for an overview and examples, cf. table I. Interestingly, TDDFT and NEGF are highly complementary, so it will be important to develop suitable combinations that allow one to overcome bottlenecks. Here we mention a recently proposed hybrid 
scheme [241], as an example.

Another important goal will have to be to use the results of TDDFT and NEGF as input to simpler approaches such as the quantum Boltzmann equation, e.g. Ref. 57. Moreover, these simulations can also be extended to longer times using the coupling to a reduced system of rate equations (DFDM) as was explained in the context of MD simulations above. In fact, in Sec. V for the computation of the secondary electron emission coefficient also a system of rate equations was derived that allows to capture the relevant degrees of freedom (electronic states of the helium projectile).

Finally, to properly capture the influence of the plasma on the solid, the above surface simulations have to be linked to fluid or kinetic simulations of the plasma, as indicated by the arrows in Fig. 7. Ultimately, an integrated modeling of the plasma and the solid surface will be required [16] to overcome the trial and error character of many experiments and to achieve a predictive modeling of the relevant processes. In Sec. IX we presented two possible approaches that are based on the quantum Boltzmann equation and nonequilibrium Green functions, within an embedding approach, respectively. Even though these have been rather simple examples and model systems, they indicate the way how such an integrated modeling can be constructed in the future.

We expect that our results will not only be of relevance for "traditional" materials embedded in a plasma such as metals or semiconductors but also for new materials. Of particular interest, in the near future, could be nanomaterials, are such as boron nitride structures or carbon nanotubes or graphene sheets and nanoribbons. For these nanomaterials both finitie size effects and electronic correlations will be of particular importance $[4,5]$ and the array of methods oulined in this article should be, in their combination, suitable to describe plasmasurface interaction with such exciting novel materials.

\section{Acknowledgements}

The authors acknowledge many fruitful discussions with our colleagues in Kiel - in particular, M. Bauer, J. Benedikt, J. Golda, B. Hartke, H. Kersten, O. Magnussen, K. Rossnagel, J. Stettner, and T. Trottenberg - with whom the present concept has been developed. EP is grateful to L. Deuchler for insightful discussions concerning TDDFT-MD simulations. MB and $\mathrm{KB}$ are grateful to A. Marini and D. Sangalli for their support in using their ab initio NEGF-code Yambo. MB is grateful to K. Becker, A. Bogaerts, P. Bruggeman, Z. Donko, J.G. Eden, U. Fantz, I. Kaganovich, M. Kushner, E. Neyts, G. Oehrlein, K. Ostrikov, and Y. Raitses for many stimulating remarks during presentation of early versions of this work.
${ }^{1}$ F. Skiff and J. Wurtele, Tech. Rep., U.S. Department of Energy, Office of Sciences (2017), report of the panel on Frontiers of Plasma Science.

${ }^{2}$ M. Meyyappan, Journal of Physics D: Applied Physics 44, 174002 (2011), URL http://stacks.iop.org/ $0022-3727 / 44 / i=17 / a=174002$.

${ }^{3}$ K. Ostrikov, E. C. Neyts, and M. Meyyappan, Advances in Physics 62, 113 (2013), https://doi.org/10.1080/00018732.2013.808047, URL https: //doi.org/10.1080/00018732.2013.808047.

4 Y.-W. Son, M. L. Cohen, and S. G. Louie, Phys. Rev. Lett. 97, 216803 (2006), URL https: / / link.aps.org/doi/10. 1103 /PhysRevLett. 97.216803.

5 D. Prezzi, D. Varsano, A. Ruini, A. Marini, and E. Molinari, Phys. Rev. B 77, 041404 (2008), URL https : / / ink . aps. org/doi/10.1103/PhysRevB.77.041404.

${ }^{6}$ I. Adamovich, S. D. Baalrud, A. Bogaerts, P. J. Bruggeman, M. Cappelli, V. Colombo, U. Czarnetzki, U. Ebert, J. G. Eden, P. Favia, et al., J. Phys. D: Appl. Phys. 50, 323001 (2017), URL http://stacks.iop.org/0022-3727/ $50 / i=32 / a=323001$

${ }^{7}$ G. J. M. Hagelaar and L. C. Pitchford, Plasma Sources Science and Technology 14, 722 (2005), URL http: / / stacks. iop. org/0963-0252/14/i=4/a=011.

8 Z. Donko and N. Dyatko, The European Physical Journal D 70, 135 (2016), ISSN 1434-6079, URL https : / / doi . org/ 10 . $1140 /$ epjd/e2016-60726-4.

9 J. Teunissen and U. Ebert, Plasma Sources Science and Technology 25, 044005 (2016), URL http://stacks.iop.org/ $0963-0252 / 25 / i=4 / a=044005$.

${ }^{10}$ M. M. Becker, H. Kählert, A. Sun, M. Bonitz, and D. Loffha- gen, Plasma Sources Science and Technology 26, 044001 (2017), URL http://stacks.iop.org/0963-0252/26/i=4/ $a=044001$.

11 A. Derzsi, I. Korolov, E. Schüngel, Z. Donkó, and J. Schulze, Plasma Sources Sci. Technol. 24, 034002 (2015), URL https://doi.org/10.1088\%2F0963-0252\%2F24\% 2F $3 \div 2 \mathrm{~F} 034002$.

12 A. V. Phelps and Z. Lj. Petrovic, Plasma Sources Sci. Technol. 8, R21 (1999).

13 P. Brault, Frontiers in Physics 6, 59 (2018), ISSN 2296-424X, URL https://www. frontiersin.org/article/10. 3389 /fphy.2018.00059.

${ }^{14}$ S. Zhao, W. Kang, J. Xue, X. Zhang, and P. Zhang, J. Phys. Condens. Matter 27, 025401 (2015).

15 K. Balzer, N. Schlünzen, and M. Bonitz, Phys. Rev. B 94, 245118 (2016), URL https: / / ink .aps .org/doi/10. 1103 /PhysRevB.94.245118.

16 This concept is based on a research project devoted to plasmasurface physics that is being developed at Kiel University and was first presented by M. Bonitz at the Conference Quo vadiscomplex plasmas, Hamburg, August 2015, and at the GEC in Bochum, October 2015.

17 D. B. Graves and P. Brault, J. Phys. D: Appl. Phys. 42, 194011 (2009).

18 E. C. Neyts and P. Brault, Plasma Processes and Polymers 14, $1600145 \quad$ (2017), https://onlinelibrary.wiley.com/doi/pdf/10.1002/ppap.201600145, URL https://onlinelibrary.wiley.com/doi/ abs/10.1002/ppap.201600145.

19 J. P. Sheehan, N. Hershkowitz, I. D. Kaganovich, H. Wang, Y. Raitses, E. V. Barbat, B. R. Weatherfor, and D. Sydorenko, 
Phys. Rev. Lett. 111, 075002 (2013).

${ }^{20}$ F. X. Bronold and H. Fehske, Phys. Rev. Lett. 115, 225001 (2015).

21 A. Sun, M. M. Becker, and D. Loffhagen, Plasma Sources Science and Technology 27, 054002 (2018), URL http: // stacks.iop.org/0963-0252/27/i=5/a=054002.

22 Y. Li and D. B. Go, Applied Physics Letters 103, 234104 (2013), https://doi.org/10.1063/1.4841495, URL https: / / doi.org/10.1063/1.4841495.

${ }^{23}$ H. von Helmholtz, Ann. Phys. (Berlin) 165, 211 (1853).

${ }^{24}$ R. L. Heinisch, F. X. Bronold, and H. Fehske, Phys. Rev. B 85, 075323 (2012).

25 G. Onida, L. Reining, and A. Rubio, Rev. Mod. Phys. 74, 601 (2002), URL https://link.aps.org/doi/10.1103/ RevModPhys.74.601.

${ }^{26}$ G. Kotliar, S. Y. Savrasov, K. Haule, V. S. Oudovenko, O. Parcollet, and C. A. Marianetti, Rev. Mod. Phys. 78, 865 (2006), URL https://link.aps.org/doi/10.1103/ RevModPhys.78.865.

27 W. M. C. Foulkes, L. Mitas, R. J. Needs, and G. Rajagopal, Rev. Mod. Phys. 73, 33 (2001), URL https : / / ink . aps . org/ doi/10.1103/RevModPhys.73.33.

28 T. Dornheim, S. Groth, and M. Bonitz, Physics Reports 744, 1 (2018), ISSN 0370-1573, URL http: //www.sciencedirect.com/science/article/ pii/s0370157318300516.

29 J.-W. Abraham, Ph.D. thesis, Kiel University, Kiel, FRG (2018), unpublished.

${ }^{30} \mathrm{M}$. Daniil, T. Carlos, and G. Vasco, Plasma Processes and Polymers 14, 1600175 (2016), https://onlinelibrary.wiley.com/doi/pdf/10.1002/ppap.201600175, URL https://onlinelibrary.wiley.com/doi/ abs/10.1002/ppap.201600175.

31 V. Guerra and J. Loureiro, Plasma Sources Science and Technology 13, 85 (2004), URL http://stacks.iop.org/ $0963-0252 / 13 / i=1 / a=011$.

32 J. W. Abraham, N. Kongsuwan, T. Strunskus, F. Faupel, and M. Bonitz, Journal of Applied Physics 117, 014305 (2015), URL http://scitation.aip.org/content/ aip/journal/jap/117/1/10.1063/1.4905255.

33 K. Fujioka, Ph.D. thesis, Kiel University, Kiel, FRG (2015), unpublished.

34 O. Polonskyi, A. M. Ahadi, T. Peter, K. Fujioka, J. W. Abraham, E. Vasiliauskaite, A. Hinz, T. Strunskus, S. Wolf, M. Bonitz, et al., The European Physical Journal D 72, 93 (2018), ISSN 1434-6079, URL https://doi.org/10. $1140 /$ epjd/e2017-80419-8.

35 L. Rosenthal, Ph.D. thesis, Kiel University, Kiel, FRG (2013), unpublished.

36 E. Runge and E. K. U. Gross, Phys. Rev. Lett. 52, 997 (1984), URL https://link.aps.org/doi/10.1103/ PhysRevLett.52.997.

37 K. Balzer and M. Bonitz, Nonequilibrium Green's Functions Approach to Inhomogeneous Systems (Springer, Berlin Heidelberg, 2013).

${ }^{38}$ N. Schlünzen and M. Bonitz, Contributions to Plasma Physics 56, 5 (2016), https://onlinelibrary.wiley.com/doi/pdf/10.1002/ctpp.201610003, URL https://onlinelibrary.wiley.com/doi/ abs/10.1002/ctpp.201610003.

39 A. Marini, C. Hogan, M. Grüning, and D. Varsano, Comp. Phys. Commun. 180, 1392 (2009).

${ }^{40} \mathrm{H}$. Jürg, Wiley Interdisciplinary Reviews: Com-

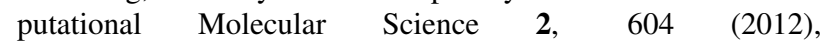

https://onlinelibrary.wiley.com/doi/pdf/10.1002/wcms.90,

URL https://onlinelibrary.wiley.com/doi/ abs/10.1002/wcms. 90.

41 A. Gross, Theoretical Surface Science (Cambridge University Press, Springer, 2009), 2nd ed.

42 M. Bonitz, J. Lopez, K. Becker, and H. Thomsen, eds., Complex Plasmas: Scientific Challnges and Technological Opportunities (Springer, 2014).

43 T. Ott and M. Bonitz, Phys. Rev. Lett. 107, 135003 (2011), URL http://link.aps.org/doi/10.1103/ PhysRevLett.107.135003.

44 J. W. Abraham, T. Strunskus, F. Faupel, and M. Bonitz, Journal of Applied Physics 119, 185301 (2016), https://doi.org/10.1063/1.4948375, URL https://doi. org/10.1063/1.4948375.

45 A. Nakano, R. K. Kalia, K. ichi Nomura, A. Sharma, P. Vashishta, F. Shimojo, A. C. T. van Duin, W. A. Goddard, R. Biswas, D. Srivastava, et al., The International Journal of High Performance Computing Applications 22, 113 (2008), https://doi.org/10.1177/1094342007085015, URL https:// doi.org/10.1177/1094342007085015.

46 S. Piana, K. Lindorff-Larsen, and D. E. Shaw, Proceedings of the National Academy of Sciences 110, 5915 (2013), ISSN 00278424, http://www.pnas.org/content/110/15/5915.full.pdf, URL http: //www.pnas.org/content/110/15/5915.

47 A. F. Voter, Phys. Rev. Lett. 78, 3908 (1997), URL https: //link.aps.org/doi/10.1103/PhysRevLett. 78 . 3908.

48 A. Laio and M. Parrinello, PNAS 99, 12562 (2002).

49 M. R. So/rensen and A. F. Voter, The Journal of Chemical Physics 112, 9599 (2000), https://doi.org/10.1063/1.481576, URL https : / /doi.org/10.1063/1.481576.

${ }^{50}$ K. M. Bal and E. C. Neyts, Journal of Chemical Theory and Computation 11, 4545 (2015), https://doi.org/10.1021/acs.jctc.5b00597, URL https: //doi.org/10.1021/acs.jctc.5b00597.

${ }^{51}$ M. Bonitz, A. V. Filinov, J. W. Abraham, and D. Loffhagen, Plasma Sources Science and Technology (2018), URL http://iopscience.iop.org/10.1088/ $1361-6595 /$ aaca75.

52 J. W. Abraham and M. Bonitz, Contributions to Plasma Physics 58, 164 (2018), https://onlinelibrary.wiley.com/doi/pdf/10.1002/ctpp.201700151, URL https://onlinelibrary.wiley.com/doi/ abs/10.1002/ctpp.201700151.

53 A. Franke and E. Pehlke, Phys. Rev. B 82, 205423 (2010).

54 A. Filinov, M. Bonitz, and D. Loffhagen, Plasma Sources Science and Technology 27, 064003 (2018), URL http: / / stacks . iop.org/0963-0252/27/i=6/a=064003.

55 M. Schwartzkopf, G. Santoro, C. J. Brett, A. Rothkirch, O. Polonskyi, A. Hinz, E. Metwalli, Y. Yao, T. Strunskus, F. Faupel, et al., ACS Appl. Mater. Interfaces 7, 13547 (2015).

56 J. W. Abraham, A. Hinz, T. Strunskus, F. Faupel, and M. Bonitz, The European Physical Journal D 72, 92 (2018), ISSN 1434-6079, URL https://doi.org/10.1140/ epjd/e2017-80426-9.

57 M. Bonitz, Quantum Kinetic Theory, Teubner-Texte zur Physik (Springer, 2016), 2nd ed.

58 A. Filinov, M. Bonitz, and D. Loffhagen, Plasma Sources Science and Technology 27, 064002 (2018), URL http: / / stacks . iop.org/0963-0252/27/i=6/a=064002.

59 M. A. Lieberman and A. J. Lichtenberg, Principles of plasma discharges and materials processing (Wiley-Interscience, New York, 2005). 
60 J. W. Rabalais, ed., Low energy ion-surface interaction (Wiley and Sons, New York, 1994).

${ }^{61}$ H. Winter, Phys. Rep. 367, 387 (2002).

${ }^{62}$ HP. Winter and J. Burgdörfer, ed., Slow heavy-particle induced electron emission from solid surface (Springer Verlag, Berlin, 2007).

63 A. V. Phelps and Z. Lj. Petrović, Plasma Sources Sci. Technol. 8, R21 (1999).

${ }^{64}$ M. Daksha, B. Berger, E. Schuengel, I. Korolov, A. Derzsi, M. Koepke, Z. Donko, and J. Schulze, J. Phys. D: Appl. Phys. 49, 234001 (2016).

65 A. Marcak, C. Corbella, T. de los Arcos, and A. von Keudell, Rev. Sci. Instrum. 86, 106102 (2015).

${ }^{66}$ M. Pamperin, F. X. Bronold, and H. Fehske, Plasma Sources Sci. Technol. (2018).

67 W. More, J. Merino, R. Monreal, P. Pou, and F. Flores, Phys. Rev. B 58, 7385 (1998).

${ }^{68}$ D. M. Newns, K. Makoshi, R. Brako, and J. N. M. van Wunnik, Phys. Scr. T6, 5 (1983).

69 A. Yoshimori and K. Makoshi, Prog. Surf. Sci. 21, 251 (1986).

${ }^{70}$ J. Los and J. J. C. Geerlings, Phys. Rep. 190, 133 (1990).

${ }^{71}$ N. P. Wang, E. A. García, R. Monreal, F. Flores, E. C. Goldberg, H. H. Brongersma, and P. Bauer, Phys. Rev. A 64, 012901 (2001).

72 D. Valdés, E. C. Goldberg, J. M. Blanco, and R. C. Monreal, Phys. Rev. B 71, 245417 (2005).

73 J. Marbach, F. X. Bronold, and H. Fehske, Eur. Phys. J. D 66, 106 (2012).

${ }^{74}$ J. Marbach, F. X. Bronold, and H. Fehske, Phys. Rev. B 86, 115417 (2012).

75 A. Iglesias-García, E. A. García, and E. C. Goldberg, Phys. Rev. B 87, 075434 (2013).

76 A. Iglesias-García, E. A. García, and E. C. Goldberg, Phys. Rev. B 90, 195416 (2014).

77 M. Pamperin, F. X. Bronold, and H. Fehske, Phys. Rev. B 91, 035440 (2015).

${ }^{78}$ M. Pamperin, F. X. Bronold, and H. Fehske, Phys. Scr. T165, 014008 (2015).

79 J. W. Gadzuk, Surf. Sci. 6, 133 (1967).

${ }^{80}$ J. W. Gadzuk, Surf. Sci. 6, 159 (1967).

${ }^{81}$ F. M. Propst, Phys. Rev. 129, 7 (1963).

82 D. R. Penn and P. Apell, Phys. Rev. B 41, 3303 (1990).

83 D. C. Langreth and P. Nordlander, Phys. Rev. B 43, 2541 (1991).

${ }^{84}$ H. Shao, D. C. Langreth, and P. Nordlander, Phys. Rev. B 49, 13929 (1994).

${ }^{85}$ H. Shao, D. C. Langreth, and P. Nordlander, in Low energy ionsurface interaction, edited by J. W. Rabalais (Wiley and Sons, New York, 1994), p. 117.

86 D. Marx and J. Hutter, Ab Initio Molecular Dynamics: Basic Theory and Advanced Methods (Cambridge University Press, 2009).

87 J. Hafner, J. Comput. Chem. 29, 2044 (2008).

88 A. Groß, Catalysis Today 260, 60 (2016).

89 T. D. Kühne, Wiley Interdisciplinary Reviews: Computational Molecular Science 4, 391 (2014).

90 M. Baer, Beyond Born-Oppenheimer: Electronic Nonadiabatic Coupling Terms and Conical Intersections (Wiley-Interscience, 2006).

91 S. Nosé, Journal of Chemical Physics 81, 511 (1984).

92 G. Henkelman and H. Jónsson, J. Chem. Phys. 113, 9978 (2000).

93 G. Henkelman, B. P. Uberuaga, and H. Jónsson, J. Chem. Phys. 113, 9901 (2000).

94 G. H. Vineyard, J. Phys. Chem. Solids 3, 121 (1957).

95 A. Laio and F. L. Gervasio, Rep. Prog. Phys. 71, 126601 (2008).

96 R. M. Martin, Electronic Structure: Basic Theory and Practical Methods (Cambridge University Press, 2004).
${ }^{97}$ K. Burke, Journal of Chemical Physics 136, 150901 (2012).

98 A. D. Becke, Journal of Chemical Physics 140, 18A301 (2014).

99 H. S. Yu, S. L. Li, and D. G. Truhlar, Journal of Chemical Physics 145, 130901 (2016).

100 P. Hohenberg and W. Kohn, Phys. Rev. 136, B864 (1964).

101 W. Kohn and L. J. Sham, Phys. Rev. 140, A1133 (1965).

102 J. Klimes and A. Michaelides, Journal of Chemical Physics 137, 120901 (2012).

103 J. Perdew, J. Chevary, S. Vosko, K. Jackson, M. Pederson, D. Singh, and C. Fiolhais, Phys. Rev. B 46, 6671 (1992).

104 J. P. Perdew, K. Burke, and M. Ernzerhof, Phys. Rev. Lett. 77, 3865 (1996).

105 J. Tao, J. P. Perdew, V. N. Staroverov, and G. E. Scuseria, Phys. Rev. Lett. 91, 146401 (2003).

106 G. Kresse and J. Furthmüller, Comp. Mat. Sci. 6, 15 (1996).

107 G. Kresse and J. Hafner, Phys. Rev. B 47, 558 (1993).

108 G. Kresse and J. Hafner, Phys. Rev. B 49, 14251 (1994).

109 G. Kresse and J. Furthmüller, Phys. Rev. B 54, 11169 (1996).

110 P. Giannozzi, S. Baroni, N. Bonini, M. Calandra, R. Car, C. Cavazzoni, D. Ceresoli, G. L. Chiarotti, M. Cococcioni, I. Dabo, et al., Journal of Physics: Condensed Matter 21, 395502 (2009).

111 P. Giannozzi, O. Andreussi, T. Brumme, O. Bunau, M. B. Nardelli, M. Calandra, R. Car, C. Cavazzoni, D. Ceresoli, M. Cococcioni, et al., Journal of Physics: Condensed Matter 29, 465901 (2017).

112 A. E. Mattsson, P. A. Schultz, M. P. Desjarlais, T. J. Mattsson, and K. Leung, Modelling Simul. Mater. Sci. Eng. 13, R1 (2005).

113 D. R. Hamann, Phys. Rev. B 40, 2980 (1989).

114 N. Trouiller and J. L. Martins, Phys. Rev. B 43, 1993 (1991).

115 M. Fuchs and M. Scheffler, Comput. Phys. Commun. 119, 67 (1999).

116 D. Vanderbilt, Phys. Rev. B 41, 7892 (1990).

117 P. E. Blöchl, Phys. Rev. B 50, 17953 (1994).

118 G. Kresse and D. Joubert, Phys. Rev. B 59, 1758 (1999).

119 A. Modinos, Progress in Surface Science 26, 19 (1987).

${ }^{120}$ R. Brako and D. M. Newns, Rep. Prog. Phys. 52, 655 (1989).

121 G. A. Kimmel and B. H. Cooper, Phys. Rev. B 48, 12164 (1993).

${ }^{122}$ H. Winter, Phys. Rep. 367, 387 (2002).

123 C. P. Race, D. R. Mason, M. W. Finnis, W. M. C. Foulkes, A. P. Horsfield, and A. P. Sutton, Rep. Prog. Phys. 73, 116501 (2010).

124 A. Wucher and A. Duvenbeck, Nucl. Instrum. Methods Phys. Res. B 269, 1655 (2011).

125 M. Lindenblatt, E. Pehlke, A. Duvenbeck, B. Rethfeld, and A. Wucher, Nuclear Instruments and Methods in Physics Research Section B: Beam Interactions with Materials and Atoms 246, 333 (2006).

126 H. Nienhaus, Surf. Sci. Rep. 45, 1 (2002).

127 D. Diesing and E. Hasselbrink, Chem. Soc. Rev. 45, 3747 (2016).

128 O. Bünermann, H. Jiang, Y. Dorenkamp, A. Kandratsenka, S. M. Janke, D. J. Auerbach, and A. M. Wodtke, Science 350, 1346 (2015).

129 A. M. Wodtke, Chem. Soc. Rev. 45, 3641 (2016).

130 S. P. Rittmeyer, J. Meyer, J. I. Juaristi, and K. Reuter, Phys. Rev. Lett. 115, 046102 (2015).

131 S. P. Rittmeyer, V. J. Bukas, and K. Reuter, Advances in Physics: X 3, 1381574 (2018).

132 M. Alducin, R. D. Muiño, and J. I. Juaristi, Progress in Surface Science 92, 317 (2017).

133 S. M. Janke, D. J. Auerbach, A. M. Wodtke, and A. Kandratsenka, The Journal of Chemical Physics 143, 124708 (2015).

134 G.-J. Kroes, M. Pavanello, M. Blanco-Rey, M. Alducin, and D. J. Auerbach, The Journal of Chemical Physics 141, 054705 (2014).

135 S. Monturet and P. Saalfrank, Phys. Rev. B 82, 075404 (2010). 
136 M. S. Mizielinski, D. M. Bird, M. Persson, and S. Holloway, J. Chem. Phys. 122, 084710 (2005).

137 M. S. Mizielinski, D. M. Bird, M. Persson, and S. Holloway, J. Chem. Phys. 126, 034705 (2007).

138 M. S. Mizielinski, D. M. Bird, M. Persson, and S. Holloway, Surf. Sci. 602, 2617 (2008).

139 M. S. Mizielinski and D. M. Bird, J. Chem. Phys. 132, 184704 (2010).

140 D. M. Bird, M. S. Mizielinski, M. Lindenblatt, and E. Pehlke, Surface Science 602, 1212 (2008).

${ }^{141}$ M. Lindenblatt, J. van Heys, and E. Pehlke, Surface Science 600, 3624 (2006).

${ }^{142}$ M. Lindenblatt and E. Pehlke, Surface Science 600, 5068 (2006).

143 M. Lindenblatt and E. Pehlke, Phys. Rev. Lett. 97, 216101 (2006).

${ }^{144}$ M. Grotemeyer and E. Pehlke, Phys. Rev. Lett. 112, 043201 (2014).

145 M. Timmer and P. Kratzer, Phys. Rev. B 79, 165407 (2009).

${ }^{146}$ S. Zhao, W. Kang, J. Xue, X. Zhang, and P. Zhang, Physics Letters A 379, 319 (2015).

${ }^{147}$ C. L. Moss, C. M. Isborn, and X. Li, Phys. Rev. A 80, 024503 (2009).

148 A. Castro, M. Isla, J. I. Martínez, and J. A. Alonso, Chemical Physics 399, 130 (2012).

149 A. V. Krasheninnikov, Y. Miyamoto, and D. Tománek, Phys. Rev. Lett. 99, 016104 (2007).

${ }^{150}$ S. Bubin, B. Wang, S. Pantelides, and K. Varga, Phys. Rev. B 85, 235435 (2012).

151 A. Ojanperä, A. V. Krasheninnikov, and M. Puska, Phys. Rev. B 89, 035120 (2014), URL https://link.aps.org/doi/ 10.1103/PhysRevB.89.035120.

152 Z. Wang, S.-S. Li, and L.-W. Wang, Phys. Rev. Lett. 114, 063004 (2015).

153 D. C. Yost, Y. Yao, and Y. Kanai, Phys. Rev. B 96, 115134 (2017).

154 A. Schleife, Y. Kanai, and A. A. Correa, Phys. Rev. B 91, 014306 (2015).

155 A. A. Correa, J. Kohanoff, E. Artacho, D. Sánchez-Portal, and A. Caro, Phys. Rev. Lett. 108, 213201 (2012).

156 M. A. Zeb, J. Kohanoff, D. Sánchez-Portal, A. Arnau, J. I. Juaristi, and E. Artacho, Phys. Rev. Lett. 108, 225504 (2012).

157 R. Ullah, F. Corsetti, D. Sánchez-Portal, and E. Artacho, Phys. Rev. B 91, 125203 (2015).

158 M. A. L. Marques, C. A. Ullrich, F. Nogueira, A. Rubio, K. Burke, and E. K. U. Gross, eds., Time-Dependent Density Functional Theory, vol. 706 of Lecture Notes in Physics (Springer, 2006).

159 M. A. L. Marques, N. T. Maitra, F. M. S. Nogueira, E. K. U. Gross, and A. Rubio, eds., Fundamentals of Time-Dependent Density Functional Theory, vol. 837 of Lecture Notes in Physics (Springer, 2012).

160 C. A. Ullrich, Time-Dependent Density-Functional Theory (Oxford University Press, 2012).

161 C. A. Ullrich and Z.-h. Yang, Braz. J. Phys. 44, 154 (2014).

162 N. T. Maitra, Journal of Chemical Physics 144, 220901 (2016).

163 E. Runge and E. K. U. Gross, Phys. Rev. Lett. 52, 997 (1984).

164 E. K. U. Gross and W. Kohn, Phys. Rev. Lett. 55, 2850 (1985).

165 M. R. Provorse and C. M. Isborn, International Journal of Quantum Chemistry 116, 739 (2016).

166 R. Nagano, K. Yabana, T. Tazawa, and Y. Abe, Phys. Rev. A 62 , 062721 (2000).

167 V. U. Nazarov, J. M. Pitarke, Y. Takada, G. Vignale, and Y.-C. Chang, Phys. Rev. B 76, 205103 (2007).

168 J. C. Tully, J. Chem. Phys. 93, 1061 (1990).

169 N. Shenvi, S. Roy, and J. C. Tully, The Journal of Chemical
Physics 130, 174107 (2009)

170 M. A. L. Marques, A. Castro, G. F. Bertsch, and A. Rubio, Comput. Phys. Commun. 151, 60 (2003).

171 N. O. Foglia, U. N. Morzan, D. A. Estrin, D. A. Scherlies, and M. C. G. Lebrero, J. Chem. Theory Comput. 13, 77 (2017).

172 G. Avendaño Franco, Ph.D. thesis, Université Catholique de Louvain (2013)

173 K. A. H. German, C. B. Weare, and J. A. Yarmoff, Phys. Rev. B 50, 14452 (1994).

174 A. Castro, H. Appel, M. Oliveira, C. A. Rozzi, X. Andrade, F. Lorenzen, M. A. L. Marques, E. K. U. Gross, and A. Rubio, physica status solidi (b) 243, 2465 (2006).

175 X. Andrade, D. Strubbe, U. De Giovannini, A. H. Larsen, M. J. T. Oliveira, J. Alberdi-Rodriguez, A. Varas, I. Theophilou, N. Helbig, M. J. Verstraete, et al., Phys. Chem. Chem. Phys. 17, 31371 (2015).

176 A. Schleife, Y. Kanai, and A. A. Correa, Phys. Rev. B 91, 014306 (2015).

177 A. A. Shukri, F. Bruneval, and L. Reining, Phys. Rev. B 93, 035128 (2016).

178 S. N. Markin, D. Primetzhofer, M. Spitz, and P. Bauer, Phys. Rev. B 80, 205105 (2009).

179 D. R. Mason, J. le Page, C. P. Race, W. M. C. Foulkes, M. W. Finnis, and A. P. Sutton, Journal of Physics: Condensed Matter 19, 436209 (2007)

180 M. K. Grotemeyer, Ph.D. thesis, Universität Kiel (2016).

181 R. D'Agosta and M. Di Ventra, Phys. Rev. B 87, 155129 (2013).

182 C. A. Ullrich, J. Chem. Phys. 125, 234108 (2006).

183 V. Kapoor, Phys. Rev. A 93, 063408 (2016).

184 N. Lorente, R. Monreal, and M. Alducin, Phys. Rev. A 49, 4716 (1994).

185 R. C. Monreal, Prog. Surf. Sci. 89, 80 (2014).

186 K. Balzer, M. Rasmussen, N. Schlünzen, J. P. Joost, and M. Bonitz, arXiv:1801.05267 (2018), submitted for publication.

187 L. Keldysh, Soviet Phys. JETP 20, 1018 (1965), (Zh. Eksp. Teor. Fiz. 47, 1515 (1964)).

188 L. Kadanoff and G. Baym, Quantum Statistical Mechanics (Benjamin, New York, 1962).

189 M. Bonitz and D. Kremp, Physics Letters A 212, 83 (1996), ISSN 0375-9601, URL http://www.sciencedirect. com/science/article/pii/0375960196000564.

190 M. Bonitz, D. Kremp, D. C. Scott, R. Binder, W. D. Kraeft, and H. S. Köhler, J. Phys.: Cond. Matt. 8, 6057 (1996), URL http: //stacks.iop.org/0953-8984/8/i=33/a=012.

191 M. Bonitz, Physics Letters A 221, 85 (1996), ISSN 0375-9601, URL http://www.sciencedirect.com/ science/article/pii/0375960196005567.

192 D. Kremp, M. Bonitz, W. Kraeft, and M. Schlanges, Annals of Physics 258, 320 (1997), ISSN 0003-4916, URL http://www.sciencedirect.com/science/ article/pii/s0003491697957031.

193 P. Danielewicz, Annals of Physics 152, 305 (1984), ISSN 0003-4916, URL http://www.sciencedirect.com/ science/article/pii/0003491684900939.

194 H. S. Köhler, Phys. Rev. C 51, 3232 (1995), URL https : / / link.aps.org/doi/10.1103/PhysRevC.51.3232.

195 L. Bányai, D. B. T. Thoai, E. Reitsamer, H. Haug, D. Steinbach, M. U. Wehner, M. Wegener, T. Marschner, and W. Stolz, Phys. Rev. Lett. 75, 2188 (1995), URL https : / / link . aps . org/ doi/10.1103/PhysRevLett.75.2188.

196 N. Kwong, M. Bonitz, R. Binder, and H. Köhler, phys. stat. sol. (b) 206, 197 (1998).

197 R. Binder, S. Köhler, and M. Bonitz, Phys. Rev. B 55, 5110 (1997). 
198 M. Bonitz, K. Balzer, and R. van Leeuwen, Phys. Rev. B 76, 045341 (2007), URL http://link.aps.org/doi/10. 1103 /PhysRevB. 76.045341.

199 K. Balzer, M. Bonitz, R. van Leeuwen, A. Stan, and N. E. Dahlen, Phys. Rev. B 79, 245306 (2009), URL http : / / link. aps.org/doi/10.1103/PhysRevB.79.245306.

200 D. Kremp, T. Bornath, M. Bonitz, and M. Schlanges, Phys. Rev. E 60, 4725 (1999), URL http://link.aps.org/doi/ 10.1103/PhysRevE.60.4725.

201 M. Bonitz, T. Bornath, D. Kremp, M. Schlanges, and W. D. Kraeft, Contrib. Plasma Phys. 39, 329 (1999), ISSN 1521-3986, URL http://dx.doi.org/10.1002/ctpp. 2150390407.

${ }^{202}$ G. Stefanucci and R. van Leeuwen, Nonequilibrium Many-Body Theory of Quantum Systems (Cambridge University Press, Cambridge, 2013).

${ }^{203}$ K. Balzer, S. Bauch, and M. Bonitz, Phys. Rev. A 81, 022510 (2010), URL http://link.aps.org/doi/10. 1103 /PhysRevA.81.022510.

${ }^{204}$ K. Balzer, S. Bauch, and M. Bonitz, Phys. Rev. A 82, 033427 (2010), URL http://link.aps.org/doi/10. 1103 /PhysRevA. 82.033427.

205 A. C.-O. Verdozzi C. Wacker A. and B. M., Journal of Physics: Conference Series 696, 011001 (2016), URL http : / / stacks.iop.org/1742-6596/696/i=1/a=011001.

206 N. Schlünzen, S. Hermanns, M. Bonitz, and C. Verdozzi, Phys. Rev. B 93, 035107 (2016), URL http: / / link.aps .org/ doi/10.1103/PhysRevB.93.035107.

${ }^{207}$ M. Bonitz, M. Scharnke, and N. Schlünzen, Contributions to Plasma Physics 58 (2018), https://onlinelibrary.wiley.com/doi/pdf/10.1002/ctpp.201700052, URL https://onlinelibrary.wiley.com/doi/ abs/10.1002/ctpp.201700052.

208 TRIM and SRIM code packages, www.srim.org.

209 S. Heese, Dielectric function of graphene with yambo (2017), bachelor thesis, Kiel University, unpublished.

210 M. Pamperin, F. X. Bronold, and H. Fehske, Phys. Rev. B 91, 035440 (2015).

211 W. Brenig, Zeitschrift für Physik B Condensed Matter 36, 81 (1979), ISSN 1431-584X, URL https: //doi.org/10. 1007 /BF01333956.

212 M. Bonitz, L. Rosenthal, K. Fujioka, V. Zaporojtchenko, F. Faupel, and H. Kersten, Contributions to Plasma Physics 52, 890 (2012), https://onlinelibrary.wiley.com/doi/pdf/10.1002/ctpp.201200038, URL https://onlinelibrary.wiley.com/doi/ abs/10.1002/ctpp.201200038.

213 W. Brenig and E. Pehlke, Prog. Surf. Sci. 83, 263 (2008).

${ }^{214}$ F. X. Bronold and H. Fehske, J. Phys. D: Appl. Phys 50, 294003 (2017).
215 I. Langmuir and H. Mott-Smith, Gen. Electr. Rev. 27, 449 (1924).

216 S. Robertson, Plasma Phys. Control. Fusion 55, 093001 (2013).

217 R. P. Brinkmann, J. Phys. D: Appl. Phys. 42, 194009 (2009).

218 R. N. Franklin, J. Phys. D: Appl. Phys. 36, R309 (2003).

219 K.-U. Riemann, J. Phys. D: Appl. Phys. 24, 493 (1991).

${ }^{220}$ L. A. Schwager and C. K. Birdsall, Phys. Fluids B 2, 1057 (1990).

221 M. D. Campanell and M. V. Umansky, Phys. Rev. Lett. 116, 085003 (2016).

222 S. Langendorf and M. Walker, Phys. Plasma 22, 033515 (2015).

223 J. P. Sheehan, N. Hershkowitz, I. D. Kaganovich, H. Wang, Y. Raitses, E. V. Barnat, B. R. Weatherford, and D. Sydorenko, Phys. Rev. Lett. 111, 075002 (2013).

${ }^{224}$ D. Sydorenko, I. D. Kaganovich, Y. Raitses, and A. Smolyakov, Phys. Rev. Lett. 103, 145004 (2009).

${ }^{225}$ F. Taccogna, S. Longo, and M. Capitelli, Phys. Plasma 11, 1220 (2004).

${ }^{226}$ P. N. Hu and S. Ziering, Phys. Fluids 9, 2168 (1966).

227 R. N. Franklin, Plasma phenomena in gas discharges (Clarendon Press, Oxford, 1976).

${ }^{228}$ M. M. Becker, G. K. Grubert, and D. Loffhagen, Eur. Phys. J. Appl. Phys. 51, 11001 (2010).

229 M. J. Kushner, J. Appl. Phys. 95, 846 (2004).

230 Y. B. Golubovskii, V. A. Maiorov, J. Behnke, and J. F. Behnke, J. Phys. D: Appl. Phys. 35, 751 (2002).

${ }^{231}$ R. Dussart, L. J. Overzet, P. Lefaucheux, T. Dufour, M. Kulsreshath, M. A. Mandra, T. Tillocher, O. Aubry, S. Dozias, P. Ranson, et al., Eur. Phys. J. D 60, 601 (2010).

${ }^{232}$ M. K. Kulsreshath, L. Schwaederle, L. J. Overzet, P. Lefaucheux, J. Ladroue, T. Tillocher, O. Aubry, M. Woytasik, G. Schelcher, and R. Dussart, J. Phys. D: Appl. Phys. 45, 285202 (2012).

233 J. G. Eden, S.-J. Park, J. H. Cho, M. H. Kim, T. J. Houlahan, B. Li, E. S. Kim, T. L. Kim, S. K. Lee, K. S. Kim, et al., IEEE Trans. Plasma Sci. 41, 661 (2013).

${ }^{234}$ P. A. Tchertchian, C. J. Wagner, T. J. Houlahan Jr., B. Li, D. J. Sievers, and J. G. Eden, Contr. Plasma Phys. 51, 889 (2011).

235 N. P. Ostrom and J. G. Eden, Appl. Phys. Lett. 87, 141101 (2005).

236 Z. Sternovsky, Plasma Sources Sci. Technol. 14, 32 (2005).

237 K.-U. Riemann, J. Phys. D: Appl. Phys. 36, 2811 (2003).

238 T. E. Sheridan and J. Goree, Phys. Fluids B 3, 2796 (1991).

239 T. V. Tsankov and U. Czarnetzki, Plasma Sources Sci. Technol. 26, 055003 (2017)

240 D. Lacroix, S. Hermanns, C. M. Hinz, and M. Bonitz, Phys. Rev. B 90, 125112 (2014), URL http: / / ink.aps.org/doi/ 10.1103 /PhysRevB.90.125112.

241 M. Hopjan, D. Karlsson, S. Ydman, C. Verdozzi, and C.-O. Almbladh, Phys. Rev. Lett. 116, 236402 (2016), URL https://link.aps.org/doi/10.1103/ PhysRevLett.116.236402. 